CARTE, STUDI E OPERE CENTRO TRENTIN DI VENEZIA

ISSN 2612-7954 (PRINT) - ISSN 2612-7946 (ONLINE)

$$
-5-
$$




\section{CARTE, STUDI E OPERE - CENTRO TRENTIN DI VENEZIA}

Il Centro documentazione e ricerca Trentin nasce a Venezia nel 2012 per iniziativa dell'Istituto veneziano per la storia della Resistenza e della società contemporanea, con lo scopo di riunire le diverse associazioni e istituti che posseggono fondi documentari relativi alla famiglia Trentin - l'esule antifascista Silvio, la moglie Beppa, i figli Giorgio, Franca e Bruno - o che su di essa promuovono ricerche ed iniziative.

\section{Director}

Cortese Fulvio, University of Trento, Italy

\section{Scientific Board}

Albanese Giulia, University of Padua, Italy Ariemma Iginio $\dagger$

Becherucci Andrea, European University Institute, Italy Bresciani Marco, University of Florence, Italy

Carnaghi Benedetta, Cornell University, United States

Casellato Alessandro, University of Venice Ca' Foscari, Italy

Cruciani Sante, Tuscia University, Italy

De Luna Giovanni, University of Turin, Italy

Epifani Guglielmo, Bruno Trentin Association, Italy

Mari Giovanni, University of Florence, Italy

Polito Pietro, Piero Gobetti Centre for Studies, Italy

Rullani Enzo, Venice International University, Italy

Sbordone Giovanni, Trentin Centre for Studies, Italy

Trentin Antonella, independent scholar, Italy

Verri Carlo, University of Palermo, Italy

Vial Eric, University of Cergy-Pontoise, France 
Norberto Bobbio

\title{
L'esempio di Silvio Trentin
}

Scritti 1954-1991

\author{
a cura di \\ Pina Impagliazzo \\ Pietro Polito
}

FIRENZE UNIVERSITY PRESS 


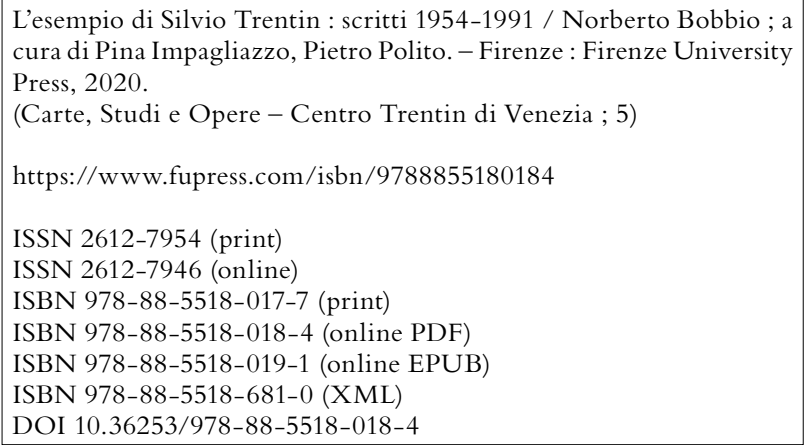

Graphic design: Alberto Pizarro Fernández, Lettera Meccanica SRLs

Con il Patrocinio del Centro studi Piero Gobetti di Torino

FUP Best Practice in Scholarly Publishing (DOI 10.36253/fup_best_practice)

All publications are submitted to an external refereeing process under the responsibility of the FUP Editorial Board and the Scientific Boards of the series. The works published are evaluated and approved by the Editorial Board of the publishing house, and must be compliant with the Peer review policy, the Open Access, Copyright and Licensing policy and the Publication Ethics and Complaint policy. Firenze University Press Editorial Board

M. Garzaniti (Editor-in-Chief), M.E. Alberti, M. Boddi, A. Bucelli, R. Casalbuoni, F. Ciampi, A. Dolfi, R. Ferrise, P. Guarnieri, R. Lanfredini, P. Lo Nostro, G. Mari, A. Mariani, P.M. Mariano, S. Marinai, R. Minuti, P. Nanni, A. Orlandi, A. Perulli, G. Pratesi, O. Roselli.

$\widehat{O}$ The online digital edition is published in Open Access on www.fupress.com.

Content license: the present work is released under Creative Commons Attribution 4.0 International license (CC BY 4.0: http://creativecommons.org/licenses/by/4.0/legalcode). This license allows you to share any part of the work by any means and format, modify it for any purpose, including commercial, as long as appropriate credit is given to the author, any changes made to the work are indicated and a URL link is provided to the license.

Metadata license: all the metadata are released under the Public Domain Dedication license (CC0 1.0 Universal: https://creativecommons.org/publicdomain/zero/1.0/legalcode).

(C) 2020 Author(s)

Published by Firenze University Press

Firenze University Press

Università degli Studi di Firenze

via Cittadella, 7, 50144 Firenze, Italy

www.fupress.com

This book is printed on acid-free paper

Printed in Italy 
Pina Impagliazzo, Pietro Polito

TESTI

SILVIO TRENTIN

SILVIO TRENTIN E LO STATO FASCISTA

LA CRISI EUROPEA E LO STATO FEDERALE

NELL'OPERA DI SILVIO TRENTIN

L'OPERA DI SILVIO TRENTIN

FEDERALISMO E LIBERTÀ

FRANÇOIS GÉNY

\section{APPENDICE}

LETTERE DI AGOSTINO ZANON DAL BO, BEPPA NARDARI TRENTIN, ALDO GAROSCI, BRUNO TRENTIN, NORBERTO BOBBIO

a cura di Pina Impagliazzo e Pietro Polito 
NORBERTO BOBBIO, SILVIO TRENTIN

E IL CENTRO STUDI PIERO GOBETTI

Frank Rosengarten

BIBLIOGRAFIA

DEGLI SCRITTI DI E SU SILVIO TRENTIN 


\title{
RINGRAZIAMENTI
}

\author{
Pietro Polito
}

Devo il mio primo accostamento a Silvio Trentin ad Anna Fiammetta Lazzarini, Raffaello Zannoner e Frank Rosengarten: un appassionamento che si è mantenuto vivo nella frequentazione e nella comunanza di idee con Luisa Bellina e Fulvio Cortese. Nei miei studi trentiniani mi è stato di grande giovamento il confronto con Corrado Malandrino e Davide Cadeddu.

Il progetto di questo libro è nato nella discussione e nella consuetudine di lavoro che, per caso o per destino, per virtù o fortuna, ho avuto con il mio maestro Norberto Bobbio. Il libro documenta e restituisce l'itinerario di vita e di studi che ha legato Bobbio a Silvio Trentin. Esso raccoglie gli scritti su Trentin da lui composti in varie occasioni, per lo più anniversari e ricorrenze, dal 1954 al 1991.

Pina Impagliazzo ha curato gli scritti di Bobbio raccolti nel volume e le lettere di Beppa Nardari Trentin, Bruno Trentin, Aldo Garosci, Agostino Zanon Dal Bo, Norberto Bobbio.

Luisa Bellina ha scritto la nota sulla famiglia Trentin che impreziosisce il volume.

Francesca Somenzari ha tradotto le pagine relative a Bobbio, Trentin e il Centro studi Piero Gobetti contenute in F. Rosengarten, Through Partisans Eyes. My Friendships, Literary Education and Political Encounters in Italy (1956-2013), Firenze University Press, Firenze 2014.

A Marina Brondino sono debitore di consigli archivistici e di quelli di una attenta lettrice.

Il libro non sarebbe mai nato senza l'invito, il consiglio, la presenza attenta di Iginio Ariemma che lo ha seguito passo dopo passo e lo ha letto nella sua ultima stesura, dandoci idealmente il si stampi. 



\title{
INTRODUZIONE. UN INCONTRO TRA DUE MAESTRI
}

\author{
Pietro Polito
}

Come scrive Norberto Bobbio in uno dei saggi compresi in questo libro, Silvio Trentin è stato una

figura eccezionale di uomo di studi e d'azione, che non dissocia mai l'impegno dello studioso da quello del militante, per il quale, riprendendo i titoli dei due famosi saggi di Max Weber, tanto la scuola quanto la politica sono insieme, e non disgiuntamente, una professione e una vocazione. Non disgiuntamente, e però non confuse. Trentin sapeva perfettamente dare alla scienza quel che è della scienza e alla politica quel che è della politica.

In questo senso Trentin è stato e rimane un esempio. Un «esempio d'indipendenza di pensiero, di energia morale indomabile, d'alta virtù civica, di fedeltà, senza compromessi né riserve, al puro ideale del diritto» (François Gény); un «esempio che si rivelò così necessario per far vivere la speranza nelle ore buie della guerra e dell'occupazione» (Jean-Pierre Vernant); «un esempio che ancora continua a ispirare i socialisti che amano la libertà» (Frank Rosengarten). La ragione fondamentale per cui Trentin è un esempio che ci parla ancora, per Bobbio, è che egli ha impersonato perfettamente con la vita e con il pensiero la concezione della politica come volontà di giustizia contrapposta alla volontà di dominio.

1. Le vite di Silvio Trentin (San Donà di Piave [Ve], 11 novembre 1885-Monastier [Tv], 12 marzo 1944) e di Norberto Bobbio (Torino, 18 ottobre 1909-9 gennaio 2004) si incrociano tra il 6 settembre 1943 e il 12 marzo 1944, quando il primo rientra in Italia, dopo gli anni dell'esilio volontario in Francia, per assumere di fatto la direzione del movimento dell'antifascismo e della Resistenza nel Veneto ${ }^{1}$ e il

${ }^{1}$ Su Silvio Trentin negli ultimi mesi della sua vita e sulla Resistenza nel Veneto: E. Brunetta, Correnti politiche e classi sociali alle origini della Resistenza nel Veneto, Neri Pozza, Vicenza 1974; A. Ventura, Padova, Laterza, Roma-Bari 1989; A.M. Preziosi (a cura di), Politica e organizzazione della Resistenza Armata. I. Atti del Comando Militare Regionale Veneto. Carteggi di esponenti azionisti (1943-1944), Neri Pozza, Vicenza 1992; A. Ventura, L'Università di Padova nella Resistenza, in «Quaderni per la storia dell'Uni- 
secondo insegna a Padova ed è attivo nelle fila del liberalsocialismo e del Partito d'azione ${ }^{2}$.

Bobbio arriva a Padova come docente di filosofia del diritto nel dicembre del 1940. Ha come assistenti Enrico Opocher (1914-2004) e Dino Fiorot (1919-2011). A poco a poco, grazie a lui, l'Istituto di Filosofia del diritto diventa un punto di riferimento per l'azionismo veneto. A 33 anni, nell'ottobre del 1942, partecipa alla riunione di fondazione del Partito d'Azione veneto tenutasi a Treviso nello studio dell'avvocato Leopoldo Ramanzini, alla presenza dei principali esponenti del partito. Tra gli altri: Antonio Giuriolo, Agostino Zanon Dal Bo, Luigi Cosattini, Ugo La Malfa ${ }^{3}$.

Bobbio conosce personalmente Trentin nell'ottobre 1943 alla prima riunione del Partito d'Azione veneto con la sua presenza. Come egli stesso ha rievocato, il ritorno di Trentin dall'esilio agita non poco le acque nelle fila del partito:

Negli incontri, in cui si ponevano le basi della organizzazione clandestina recava il contributo della sua esperienza, delle sue idee a lungo maturate e profondamente radicate nel suo spirito, e della sua ferma coscienza. Lo riconoscemmo immediatamente come la guida che avevamo cercata. Ricordo la serenità del suo volto che incuteva rispetto, quella austerità del comportamento che dava e chiedeva fiducia, il passo sicuro che

versità di Padova», 28, 1995, pp. 157-172; V. Marangon, Resistenza padovana tra memoria e storia, Centro studi Ettore Luccini, il Poligrafo, Padova 1995; R. Biondo, M. Borghi (a cura di), Giustizia e libertà e Partito d'azione a Venezia e dintorni, Nuova Dimensione, Portogruaro 2005; M. Biason, Un soffio di libertà. La Resistenza nel basso Piave, Nuova Dimensione, Portogruaro 2007; E. Brunetta, Il ritorno dall'esilio di Silvio Trentin. Il Partito d'azione tra destra e sinistra, in I. Ariemma e L. Bellina (a cura di), Bruno Trentin. Dalla guerra partigiana alla CGIL, Ediesse, Roma 2008, pp. 129-134; I. Ariemma (a cura di), Bruno Trentin tra il Partito d'Azione e il Partito Comunista. Gli anni dell'Università di Padova. 1943-1949, con la tesi di laurea e una lettera inedita a Gaetano Salvemini, Ediesse, Roma 2009; G. Bobbo, Silvio Trentin e la nascita della Resistenza nel Veneto: settembre-novembre 1943, in Pensare un'altra Italia. Il progetto politico di Silvio Trentin, Atti del Convegno, 15 gennaio 2011 (Teatro Eden, Treviso), Istresco-Iveser-Anpi, Treviso 2012, pp. 63-79; C. Verri (a cura di), I Trentin a Mira nella Resistenza, Atti del Convegno, ottobre 2011, Mira, ANPI, sezione di Mira (Venezia), Mira 2013; I. Ariemma, «Tempo perduto. Ora all'opra». Il ritorno alla libertà di Silvio e Bruno Trentin, in Id., La sinistra di Bruno Trentin. Elementi per una biografia, Ediesse, Roma 2014, pp. 39-50.

2 B. Pastore, G. Zaccaria (a cura di), Norberto Bobbio. Gli anni padovani, Padova University Press, Padova 2010 e M.G. Losano, Norberto Bobbio. Una biografia culturale, Carocci, Roma 2018, pp. 63 ss.

${ }^{3}$ Bobbio ha raccontato di questa riunione in Il primo incontro nel ' 42 , in G. Spadolini (a cura di), Per Ugo La Malfa, Edizioni della Voce, [Roma 1980], p. 49 e in Id., Autobiografia, a cura di A. Papuzzi, Laterza, Roma-Bari 1997, pp. 51-52. Vedi anche: G. Spadolini, Ugo La Malfa studente nella Venezia di Trentin e di Luzzatto, con due lettere inedite di Gino Luzzatto, discorso pronunciato a Ca' Foscari per l'inaugurazione del $131^{\circ}$ anno accademico dell'Istituto, 13 febbraio 1989, Centro Studi Nuova Ricerca, sl, sd. 
era il segno di una conquistata calma interiore e di un consapevole coraggio. La sua presenza ci offriva un incitamento per il presente e una certezza per l'avvenire ${ }^{4}$.

L'incontro tra $\mathrm{i}$ due avviene all'Istituto di Filosofia del diritto dove Bobbio insegnava. C'è un episodio che mi pare possa illuminare il carattere di entrambi. Dalla testimonianza di Zanon Dal Bo, sappiamo che le idee nuove portate da Trentin suscitarono interesse ma non furono immediatamente comprese. Mi viene da pensare che Bobbio non sia estraneo alla decisione di costituire un gruppo di studio per approfondirle, formato da lui, Dal Pra e dallo stesso Zanon Dal Bo5.

Con il figlio Bruno, Trentin partecipa a un evento straordinario: l'inaugurazione del $722^{\circ}$ anno dell'università di Padova, il 9 novembre 1943. Sull'episodio abbiamo le testimonianze di Bobbio e di Bruno Trentin. Entrambi ammirano il coraggio con cui Marchesi affrontò i fascisti armati e il loro capo. Bobbio ha scritto che Trentin, confuso tra la folla assistette, condottovi da un amico, all'apertura dell'anno accademico, «quando Concetto Marchesi sfidò gli effimeri e torvi padroni inaugurando l'anno degli studi in nome dell'Italia dei lavoratori» ${ }^{6}$. Toni analoghi vengono usati dal giovane Trentin:

In mezzo ai docenti, ai presidi, si è avanzato un piccolo uomo col mantello d'ermellino: era Concetto Marchesi, che si diresse direttamente verso il palco dove parlava il capo di questo manipolo di fascisti, lo prese per la collottola e lo buttò giù dal palco letteralmente di fronte allo stupore attonito degli altri fascisti e di fronte all'entusiasmo di questa folla di studenti che aspettava un segno ${ }^{7}$.

La sera del 19 novembre 1943 Trentin fu arrestato e rinchiuso col figlio Bruno nel carcere dei Paolotti. Uscito di prigione, il suo impe-

${ }^{4}$ N. Bobbio, Italia civile. Ritratti e testimonianze, Manduria-Bari-Perugia 1964; nuova ed. Passigli, Firenze 1986, p. 265.

${ }^{5}$ Le opere di Trentin circolavano all'Istituto di Filosofia del diritto di Padova: «Silvio Trentin veniva da Bobbio presentato come uno studioso costituzionalista che aveva elaborato delle idee estremamente interessanti e moderne». Cfr. D. Fiorot, Il mio ricordo di Norberto Bobbio negli anni 1943-1946, in Pastore, Zaccaria (a cura di), Norberto Bobbio. Gli anni padovani, cit., p. 44.

${ }^{6}$ N. Bobbio, Silvio Trentin, in Italia civile, cit., p. 265 . Vedi anche Id., La mia Italia, a cura di P. Polito, Passigli, Firenze 2001, pp. 199-201.

${ }^{7}$ F. Giraldi (a cura di), L'appello contro il fascismo rivolto da Concetto Marchesi agli studenti nella testimonianza di Bruno Trentin, in Ariemma (a cura di), Bruno Trentin tra il Partito d'Azione e il Partito Comunista. Gli anni dell'università di Padova 1943-1949, cit., pp. 93-94, dove vedi anche la testimonianza di Franco Busetto, pp. 53-55. Vedi inoltre Id., La mia guerra partigiana, a cura di G. Albanese, in Ariemma e Bellina (a cura di), Bruno Trentin. Dalla guerra partigiana alla CGIL, cit., p. 62, che ripropone la testimonianza di Busetto, pp. 136-139. 
gno fu interrotto dall'aggravarsi della malattia. Dal 6 dicembre all'11 febbraio $1944 \mathrm{fu}$ ricoverato all'ospedale "Elena di Savoia" di Treviso. A causa dei bombardamenti fu trasferito alla clinica Carisi nella cittadina di Monastier, tra Treviso e San Donà del Piave. Morì laicamente il 12 marzo 1944.

Lo stesso Bobbio è tornato più volte sul rapporto tra il fascismo e la cultura $^{8}$, sul suo rapporto personale col fascismo con forti accenti di autocritica personale 9 , sulla sua partecipazione come uomo di cultura alla Resistenza che si sviluppa prevalentemente nel Veneto ${ }^{10}$. La sua esperienza partigiana si conclude con la partecipazione alle elezioni del 2 giugno 1946 nelle liste del Partito d'Azione, nella circoscrizione Verona-PadovaVicenza-Rovigo. Egli è l'unico non veneto della lista e viene candidato perché, allora docente di Filosofia del diritto a Padova, appunto, era stato tra i fondatori del Partito nel Veneto nel 1942. Bobbio tiene anche alcuni comizi, a Vicenza e a Valdagno il 12 e il 26 maggio 1946, ma risulta non eletto con 1599 voti, al secondo posto dopo Egidio Meneghetti che ne ottenne 3250. Nell'Autobiografia definisce la prova elettorale del Partito una sconfitta "catastrofica in tutto il Paese» e la sua «un fiasco incredibile» tanto che disse a sé stesso: «basta, la mia vita politica è finita».

2. Il debito contratto col compagno di lotta negli anni della prova decisiva non si cancella con la morte di Trentin, anzi si prolunga in varie riprese negli anni successivi ${ }^{12}$. Come ha scritto Moreno Guerrato:

Di Silvio il grande filosofo era stato amico e compagno nella Resistenza a Padova e a Trentin, in quei giorni di lotta, Bobbio aveva guardato infatti come suo punto di riferimento. Entrambi militavano nel Partito d'Azione ed entrambi conobbero a Padova il carcere, nel 1943, sia pure

\footnotetext{
${ }^{8}$ Si veda in primo luogo il saggio Se sia esistita una cultura fascista (1975), in Id., Il dubbio e la scelta. Intellettuali e potere nella società contemporanea, La Nuova Italia Scientifica, Roma 1993, pp. 101-111. I suoi principali saggi sul tema si trovano in Id., Dal fascismo alla democrazia. I regimi, le ideologie, le figure e le culture politiche, a cura di M. Bovero, Baldini\&Castoldi, Milano 1997.

${ }_{9}$ Bobbio, Autobiografia, cit., pp. 28-40. Segnalo un testo poco conosciuto in cui egli ricostruisce in modo asettico, ma senza sconto alcuno verso sé stesso, la vicenda della lettera «disonorevole» da lui scritta a Benito Mussolini l'8 luglio 1935: Id., «Son cose note, ma io non posso dimenticarle», «Mezzosecolo», 11, Annali 1994-1996, pp. 217- 232.

${ }^{10}$ A. Ventura, Bobbio nella Resistenza nel Veneto, in Pastore, Zaccaria (a cura di), Norberto Bobbio. Gli anni padovani, cit., pp. 17-38; ora in Id., Intellettuali. Cultura e politica tra fascismo e antifascismo, Donzelli, Roma 2017, pp. 193-210. Si veda: Bobbio, Autobiografia, cit., pp. 41-78 e Id., Eravamo ridiventati uomini. Testimonianze e discorsi sulla resistenza in Italia, a cura di P. Impagliazzo e P. Polito, Einaudi, Torino 2015.

11 Bobbio, Autobiografia, cit., p. 81.

12 G. Gangemi, Bobbio e Trentin tra Resistenza e filosofia del diritto, in Pastore, Zaccaria (a cura di), Norberto Bobbio. Gli anni padovani, cit., pp. 53-92.
} 
in momenti diversi. Nonostante la differenza d'età - 24 anni - devono essersi capiti subito perché Trentin e Bobbio quegli ideali in cui credevano li seguirono veramente nella vita ${ }^{13}$.

Bobbio inizia a occuparsi di Trentin in occasione del decennale della morte. Su invito di Enrico Opocher, egli tiene la commemorazione organizzata a Venezia il 14 marzo 1954, avvalendosi dei materiali e delle notizie che gli forniscono Beppa Nardari e Bruno Trentin, Agostino Zanon Dal Bo e Aldo Garosci ${ }^{14}$. In linea generale, per Bobbio il pensiero politico di Trentin rappresenta da un lato una delle versioni dell'ideologia della Resistenza: il comunismo liberale inteso come una terza via oltre il capitalismo e il comunismo ${ }^{15}$, dall'altro appartiene a pieno titolo alla storia del federalismo ${ }^{16}$.

L'impegno di Bobbio per Trentin è strettamente connesso al lavoro svolto dal Centro studi Piero Gobetti, di cui egli è Presidente dal 1962 al 1993. Fin dai primi anni Sessanta, per iniziativa di Ada Gobetti e Beppa Trentin e successivamente di Carla Gobetti e Franca Trentin si costituisce il «Fondo Silvio Trentin del Centro studi Piero Gobetti di Torino ${ }^{17}$. Come ha osservato Silvana Barbalato, nell'attività del Centro studi sull'eredità di Trentin «si rintraccia la forte necessità di recuperare alla memoria collettiva gli anni dell'antifascismo», un'opera che "significava anche riportare alla luce quella dimensione umana che è il valore più profondo dell'antifascismo, la testimonianza di quell'umanesimo che il fascismo aveva combattuto con i suoi delitti politici» ${ }^{18}$.

13 M. Guerrato, I trent'anni del Centro Studi e Ricerca "Silvio Trentin" (1974-2004), in Id. (a cura di), L'antifascismo italiano tra le due guerre: alla ricerca di una nuova unità, Atti del Seminario di studi italo-francese, Jesolo 2-3 aprile 2004, Introduzione di G. Paladini, Centro Studi e Ricerca "Silvio Trentin", Jesolo 2005, p. 39.

$14 \mathrm{Si}$ veda la corrispondenza in appendice a questo volume. Nel suo archivio personale sono conservati il testo dattiloscritto del discorso di Egidio Meneghetti, apparso subito dopo la morte nel foglio clandestino "Fratelli d'Italia», fogli 7, e quello della commemorazione fatta da Emilio Lussu a Roma nel 1944, fogli 4. Attraverso le sue schede di lavoro, è possibile ricostruire quali sono state le letture che egli ha fatto per la preparazione del discorso (Centro studi Piero Gobetti - Archivio Norberto Bobbio, ANB 1272). Il ritratto del 1954, pubblicato in N. Bobbio, Italia civile, pp. 249-266, apre questo volume.

15 N. Bobbio, Profilo ideologico del Novecento (1969), Garzanti, Milano 1990, pp. 189-191.

16 Id., Il federalismo nel dibattito politico e culturale della Resistenza, in L'idea dell'unificazione europea dalla Prima alla Seconda guerra mondiale, a cura di S. Pistone, Fondazione Luigi Einaudi, Torino 1975, pp. 234-235.

17 C. Malandrino, Il Fondo Silvio Trentin del Centro studi Piero Gobetti di Torino, «Movimento operaio e socialista», XI (1), 1988, pp. 139-148; poi in Id., Silvio Trentin pensatore politico antifascista, rivoluzionario, federalista, Lacaita, Manduria-Bari-Roma 2007.

18 S. Barbalato, Note sull'eredità di Silvio Trentin tra Venezia e Torino, in F. Cortese (a cura di), Liberare e federare. L'eredità intellettuale di Silvio Trentin, Atti del Convegno tenutosi a Venezia il 5 dicembre 2014, in occasione del settantesimo anniversario della morte, "Carte studi opere Centro Trentin di Venezia”, vol.4, Firenze University Press, Firenze 2016, pp. 267 e 270. 
La prima iniziativa editoriale promossa dal Centro Gobetti è il volume di Trentin, Scritti inediti. Testimonianze e studi, a cura di Paolo Gobetti con contributi di Emilio Lussu e Hans Werner Tobler, presentato da Bobbio il 26 febbraio 1972 all'assemblea dei soci. Nella prefazione al volume Bobbio scrive:

Fedele al compito originario e costitutivo di recare nuovi e specifici contributi alla conoscenza di quel particolare aspetto della storia italiana che è più direttamente legato alla lotta antifascista, il Centro studi Piero Gobetti intende con questo volume [...] colmare almeno in parte una lacuna e offrire agli studiosi un materiale sinora sconosciuto su un episodio della Resistenza europea ${ }^{19}$.

Nella storia della fortuna di Trentin e del ruolo che in essa Bobbio vi ha avuto, un posto di primo piano è occupato da Raffaello Zannoner. Il cospicuo carteggio tra i due è una fonte preziosa ${ }^{20}$. La prima lettera è del 2 settembre 1974. Bobbio si rallegra «nell'apprendere che vi sono iniziative in corso per far conoscere l'opera, quasi del tutto ignorata di Silvio Trentin». Aggiunge che i suoi contatti con lui «furono pochi e di breve durata». Ricorda di averne parlato in Italia civile (1964) e nel Profilo ideologico del Novecento (1969). Inoltre, afferma che si tratta di un autore da studiare "più a fondo» e auspica la pubblicazione di «alcune sue opere dimenticate e introvabili» ${ }^{21}$.

A trent'anni dalla morte, Bobbio torna su Trentin con la commemorazione tenuta il 9 novembre 1974 a San Donà di Piave. Una eco della manifestazione si avverte nella lettera che il 18 novembre l'allora sindaco Massimiliano Orlando gli scrive: «È stata per noi una giornata indimenticabile; come $\mathrm{mi}$ ha scritto il senatore Nenni nella lettera di adesione alla manifestazione, nessuno meglio di Norberto Bobbio avrebbe potuto ricordare l'opera di Trentin $\nu^{22}$.

${ }^{19}$ S. Trentin, Scritti inediti. Testimonianze. Studi, contributi di E. Lussu e H. W. Tobler, a cura di P. Gobetti, Guanda, Parma 1972, p X. Cfr., ANB 1272, p. 9. Il volume ha una lunga gestazione come emerge da una lettera di Franca Trentin a Paolo Gobetti del 23 marzo 1969: "Aspetto con impazienza la sudata pubblicazione su mio padre». Cfr. S. Barbalato, Note sull'eredità di Silvio Trentin tra Venezia e Torino, cit., p. 269.

${ }^{20}$ Nell'Archivio Bobbio è conservato il carteggio completo tra Bobbio e Zannoner dal 2 settembre 1974 al 4 febbraio 1991 con le minute delle lettere di Bobbio e le trascrizioni degli originali conservati presso il Centro studi e ricerca Silvio Trentin di Jesolo. La ricostruzione del carteggio è stata realizzata grazie all'interessamento di chi scrive.

${ }^{21}$ Lettera manoscritta su carta intestata «Università degli Studi di Torino Facoltà di Scienze Politiche di Torino", 2 settembre 1974, in fotocopia (ANB 1272).

${ }^{22}$ Lettera dattiloscritta su carta intestata: "Città di San Donà di Piave", con data 18.11.1974, con firma autografa. ANB 1272. Cfr. N. Bobbio, La crisi europea e lo stato federale nell'opera di Silvio Trentin, in "Città e Regione», I (8), ottobre 1975, pp. 200-212. Ora in questo volume. 
Bobbio non partecipa al convegno L'attualità del pensiero di Silvio Trentin nell'ambito della lotta antifascista unitaria per una Europa democratica, tenutosi a Jesolo il 20 aprile 1975, cui prendono parte, tra gli altri, Giannantonio Paladini, Hans Werner Tobler, Leo Valiani. In una lettera del 23 marzo 1975 a Zannoner, egli invitava a «uscire dalle commemorazioni e entrare nella ricerca storica» e si augurava che il convegno rappresentasse «un inizio di questa nuova strada» ${ }^{23}$. L'anno successivo, nella lettera del 1 maggio 1976, Bobbio commenta positivamente gli atti del Convegno e afferma che Trentin è uno scrittore, giurista e politico, «che ha lasciato tracce che non dovremmo lasciar cancellare dal tempo $»^{24}$.

3. Merita di essere seguita con attenzione la vicenda della (non) pubblicazione di La Crise du Droit et de l'État (1935). Dopo avere lodato la "costante attenzione e devozione» di Zannoner per Trentin, l'8 giugno 1975 Bobbio manifesta interesse per La crise «l'opera di Trentin, che credo teoricamente più importante», «un'opera ancora da studiare»" 25 . Il 9 dicembre dello stesso anno lo ringrazia di avergli inviato una copia del «rarissimo libro", che, su suggerimento di Carla Gobetti, si propone di riesaminare a distanza di anni, dopo averlo letto una prima volta nel lontano 1954, ai fini di una eventuale pubblicazione in Italia ${ }^{26}$.

Tuttavia, Bobbio darà un parere negativo sulla pubblicazione di $\mathrm{La}$ Crise come emerge, tra l'altro, da uno scambio con Federico Codignola. Quest'ultimo, con una lettera del 22 giugno 1976, a nome della casa editrice La Nuova Italia, gli aveva chiesto un giudizio sulla proposta di traduzione del libro avanzatagli da Zannoner ${ }^{27}$. A Bobbio (8 luglio 1976) l'opera sembra "un po' invecchiata». Gli autori esaminati da Trentin - tra cui Santi Romano e lo stesso Kelsen (ciò colpisce perché Bobbio si considera e deve essere considerato un kelseniano) -, a suo giudizio, «rappresentano tendenze fuori dall'orizzonte critico contemporaneo». Per queste ragioni egli ritiene che non si farebbe «un buon servizio alla memoria di Trentin se ci avventurassimo nella traduzione di quest'o-

${ }^{23}$ Lettera dattiloscritta su carta non intestata, Torino, 23 marzo 1975, in fotocopia, (ANB 1272). Cfr., A. Colombo, Una Resistenza che guardava all'Europa, "Corriere della sera", mercoledì 23 aprile 1975, p. 3. In Atti del Convegno di studio su Silvio Trentin, Jesolo 20 aprile 1975, Neri Pozza, Vicenza 1976 è compresa la conferenza tenuta a San Donà di Piave il 9 novembre 1974, pp. 109-123.

${ }^{24}$ Lettera dattiloscritta e manoscritta su carta intestata «66, via Sacchi - 10128 Torino",1 maggio 1976 (ANB 1272).

${ }^{25}$ Lettera dattiloscritta e manoscritta su carta intestata "Accademia Nazionale dei Lincei», 8 giugno 1975 (ANB 1272).

${ }^{26}$ Lettera dattiloscritta o manoscritta su carta intestata «Università degli Studi di Torino Facoltà di Scienze Politiche di Torino», 9 dicembre 1975 (ANB 1272).

${ }^{27}$ Lettera dattiloscritta di Federico Codignola su carta intestata «La Nuova Italia Editrice», 22 giugno 1976 (ANB 1272). 
pera»: «in questi anni - aggiunge - molta acqua è passata sotto i ponti della teoria del diritto e la mia attuale impressione è che il libro mostra molte rughe» ${ }^{28}$.

In realtà, Bobbio anticipa a Codignola i contenuti della lettera del 5 agosto 1976 a Zannoner: «L'opera è notevole per la ricchezza della documentazione, per l'analisi critica della principale letteratura giuridica del tempo, ma a mio parere non reggerebbe a una traduzione oggi, a quarant'anni dalla pubblicazione, perché prevale di gran lunga la parte critica sulla parte costruttiva». Per lui, La crise è "un'opera importante» nella formazione del pensiero del suo autore, "ma un po' troppo “datata"»"

Piuttosto Bobbio sembra interessato alla realizzazione di una monografia su Trentin. Infatti, egli apprezza il lavoro del giovane Moreno Guerrato. In una lettera del 23 settembre 1977, questi con «trepidazione» presenta la sua ricerca all'autorevole interlocutore, temendo «un'eventuale valutazione negativa» ${ }^{30}$. La risposta invece è molto lusinghiera:

Non le faccio dei complimenti di rito. Ho una certa pratica di lavori di questo genere: credo di aver imparato a distinguere le tesi ben fatte dalle tesi malfatte. Lei ha lavorato con molta serietà e, quel che non guasta, con molta passione. Ne è venuto fuori un saggio originale per il materiale raccolto, e interessante anche per il modo con cui è stato scritto; vivace e senza pedanteria ${ }^{31}$.

Un capitolo importante del nostro racconto è l'incontro tra Bobbio e Frank Rosengarten, che ho conosciuto personalmente e ho avuto modo di apprezzare per le sue qualità umane e di storico controcorrente. Lo studioso americano ha soggiornato a lungo a Torino nella seconda metà degli anni Sessanta e ha svolto ricerche sistematiche nell'archivio del Centro studi Piero Gobetti. Egli ha ricostruito il suo rapporto con il nostro Paese nel volume Through Partisans Eyes. My Friendships Literarry Education and Political Encounters in Italy (1956-2013), dove di Bobbio, tra l'altro, scrive:

Mi resi subito conto che Bobbio era un uomo semplice, senza alcuna presunzione (non si dava arie), e allo stesso tempo lucidissimo e del tutto sincero nell'esprimere le sue opinioni, dotato di un senso dello humor

${ }^{28}$ Lettera dattiloscritta su carta non intestata, Torino 8 luglio 1976, in fotocopia.

${ }^{29}$ Lettera dattiloscritta su carta intestata «66, via Sacchi - 10128 Torino», 5 agosto 1976 (ANB 1272). Sulla vicenda della pubblicazione de La crise vedi oltre.

${ }^{30}$ Lettera manoscritta su carta intestata: «GM» datata San Donà di Piave, 23 settembre 1972 (ANB 1272).

${ }^{31}$ La minuta della risposta di Bobbio, su carta intestata: «Università degli studi di Torino", Torino, 22 novembre 1977, è conservata insieme alla lettera di Guerrato (ANB 1272). 
che conferiva al suo modo di parlare un che di piacevolmente piccante. Trovammo un terreno comune nel capitolo che Bobbio aveva dedicato in «Italia Civile» al giurista Trentin, la cui vita e il cui lavoro mi avrebbero occupato per il successivo decennio ${ }^{32}$.

A Rosengarten si deve una biografia di Trentin che rimane insuperata $^{33}$. Nella già citata lettera dell' 8 luglio $1976 \mathrm{a}$ Codignola Bobbio segnala all'editore che Rosengarten ha scritto «una eccellente biografia di Trentin dopo accuratissimi studi», aggiungendo: «Sulla sua serietà non ho alcun dubbio ${ }^{34}$. Inoltre, in una lettera del 12 dicembre 1976, informa Zannoner di avere interessato al riguardo La Nuova Italia: «Sarebbe un peccato che un lavoro di tanta importanza non riuscisse a trovare un editore ${ }^{35}$.

Le difficoltà incontrate nella pubblicazione del libro emergono dalla corrispondenza con Rosengarten. Questi, in una lettera del 17 aprile 1975,

${ }^{32}$ F. Rosengarten Through Partisans Eyes. My Friendships Litterary Education and Political Encounters in Italy (1956-2013), Firenze University Press, Firenze 2014, p. 53. In appendice sono pubblicate le pagine relative a Bobbio, Trentin e il Centro studi Piero Gobetti nella traduzione di Francesca Somenzari.

${ }^{33}$ F. Rosengarten, Silvio Trentin dall'interventismo alla Resistenza, Feltrinelli, Milano 1980. Segnalo le monografie pubblicate in seguito su Trentin: M. Guerrato, Silvio Trentin un democratico all'opposizione, Vangelista, Milano 1981; G. Gangemi, Federalisti contro. Da Althusius a Silvio Trentin, prefazione di D. Fiorot, Edizioni Sapere, Padova 1997; C. Malandrino, Silvio Trentin pensatore politico antifascista, rivoluzionario, federalista, Lacaita, Manduria 2007; P. Arrighi, Silvio Trentin un Européen en Résistance (1919-1943), Loubatières, Portet-Sur-Garonne 2007; F. Cortese, Libertà individuale e organizzazione pubblica in Silvio Trentin, FrancoAngeli, Milano 2008; C. Verri, Guerra e libertà. Silvio Trentin e l'antifascismo italiano (1936-1939), XL edizioni, Roma 2011. A Trentin sono stati dedicati numerosi convegni: Silvio Trentin e la Francia, Colloquio internazionale tenutosi a Parigi l'8 e il 9 febbraio 1985, prefazione di G. Paladini, Marsilio, Venezia 1991; A. Lotto (a cura di) Lo stato del federalismo, Atti del Colloquio di storia, Jesolo 1994, introduzione di G. Paladini e A. Ventura, Centro di ricerca Silvio Trentin di Jesolo, Treviso 1998; Pensare un'altra Italia. Il progetto politico di Silvio Trentin, Atti del Convegno, 15 gennaio 2011 (Teatro Eden, Treviso), Istresco-Iveser-Anpi, Treviso 2012; M. Guerrato (a cura di), L'antifascismo italiano tra le due guerre: alla ricerca di una nuova unità, Atti del Seminario di studi italo-francese, introduzione di G. Paladini, Jesolo 2-3 aprile 2004, Centro Studi e Ricerca "Silvio Trentin", Jesolo 2005; D. Cadeddu (a cura di), Dalla libertà al federalismo. Silvio Trentin tra storia e teoria politica, Biblion edizioni, Milano 2010; Pensare un'altra Italia. Il progetto politico di Silvio Trentin, Atti del Convegno, 15 gennaio 2011 (Teatro Eden, Treviso), Istresco-Iveser-Anpi, Treviso 2012; F. Cortese (a cura di), Liberare e federare. L'eredità intellettuale di Silvio Trentin, Atti del Convegno tenutosi a Venezia il 5 dicembre 2014, in occasione del settantesimo anniversario della morte, "Carte studi opere Centro Trentin di Venezia", vol. 4, Firenze University Press, Firenze 2016; Id. (a cura di), Resistenza e diritto pubblico, Atti del Convegno tenutosi a Venezia, presso Iveser (Istituto veneziano per la Storia della Resistenza), il 30 maggio 2014, "Carte studi opere Centro Trentin di Venezia", vol. 3, Firenze University Press, Firenze 2016.

${ }^{34}$ ANB 1272.

${ }^{35}$ Lettera autografa su carta intestata «Università degli Studi di Torino, Facoltà di Scienze Politiche di Torino»,12 dicembre 1976 (ANB 1272). 
aveva annunciato a Bobbio che, dopo sette anni di lavoro, aveva concluso la biografia trentiniana, comunicandogli di avere ricevuto solo risposte negative dagli editori americani e informandosi sulle possibilità di proporre la pubblicazione del volume in Italia. Lo studioso si rivolge a Bobbio, «per tante ragioni, fra cui, e principalmente, il nostro comune senso di rapporto personale con Trentin e con la causa per la quale ha combattuto $»^{36}$.

Il rapporto tra Bobbio e Rosengarten non è solo quello tra due studiosi. Essi si sono conosciuti e hanno avuto modo di frequentarsi durante le ricerche svolte dall'americano al Centro Gobetti. Si spiegano così gli spunti di natura personale e di analisi politica presenti nelle loro lettere. Il richiamo a Trentin, 1'apostolo del socialismo democratico', si mescola sovente con l'apprensione per quanto accadeva in Italia:

Le gravi preoccupazioni per la difficile situazione politica ed economica del nostro paese - scrive a Rosengarten il 6 giugno 1975 - mi impediscono di lavorare con serenità. Se segue i giornali italiani, avrà visto che si è scatenata in questi ultimi anni la violenza politica, che era sconosciuta sino a qualche anno fa, e che ci riporta con presagi sinistri al «biennio rosso»e allo squadrismo fascista ${ }^{37}$.

4. L'impresa più importante e più significativa nella storia della fortuna di Trentin è il progetto delle Opere scelte che ha avuto una lunga gestazione e muove i primi passi nella seconda metà degli anni Settanta. La prima riunione avvenuta all'Università Ca' Foscari risale al 16 novembre 1977. Ad essa avrebbe dovuto seguirne una successiva presso il Centro studi Piero Gobetti. Forse l'impulso decisivo viene dalla lettera di Alessandro Janna, da San Donà di Piave il 16 agosto 1978, a Bobbio, Enrico Opocher, Giannantonio Paladini, Angelo Ventura. Il più anziano invita i meno anziani a «dar corso ai propositi», con l'aiuto del giovane neolaureato Moreno Guerrato e con il coordinamento di Zannoner, "creatore ed animatore» di tante iniziative trentiniane ${ }^{38}$.

Un piano iniziale della pubblicazione delle opere viene elaborato da Guerrato e da Paladini (Jesolo, 11 novembre 1979) ${ }^{39}$. Il Comitato per la

${ }^{36}$ Lettera dattiloscritta, 17 aprile 1975 (ANB 3390).

${ }^{37}$ La copia della risposta di Bobbio a Rosengarten si trova in ANB 3390.

${ }^{38}$ Lettera dattiloscritta su carta intestata «San Donà di Piave», 16 agosto 1978 (ANB 1272).

${ }^{39}$ Lettera dattiloscritta su carta intestata: «Biblioteca comunale - 30016 Jesolo - Piazza della Repubblica; Centro di ricerca "Silvio Trentin" per la documentazione e lo studio dell'antifascismo e la Resistenza nel Veneto" datata Jesolo, 11 gennaio 1979. Il piano viene inviato a C. Gobetti - Torino; F. Trentin Baratto - Venezia; N. Bobbio - Torino; M. Berengo - Venezia; A. Janna - Torino; E. Opocher - Padova; F. Rosengarten - New York; A. Ventura - Padova; e p.c. a M. Guerrato-S. Donà di Piave e G. Paladini - Venezia (ANB 1272). 
pubblicazione degli scritti di Trentin, promosso dal Centro Gobetti e dal Centro di ricerca Silvio Trentin, si riunisce a Torino il 5 maggio 1979, in via Fabro 6. Vi partecipano Bobbio, Carla e Paolo Gobetti, Alessandro Janna, Leopoldo Ramanzini, Fausto Salvatore, Franca Trentin Baratto, Angelo Ventura, Raffaello Zannoner. Si decide di concepire l'edizione delle opere come opera scelta e non come opera omnia ${ }^{40}$. L'editore viene successivamente individuato nella Marsilio di Venezia.

Il primo volume delle Opere scelte è Dallo Statuto albertino al regime fascista, a cura di Alessandro Pizzorusso ${ }^{41}$, che viene presentato da Bobbio il 23 gennaio 1984 a Venezia, nell'Aula Magna "Silvio Trentin" di Ca' Dolfin. Il 27 gennaio 1984 Zannoner gli scrive: "La sua "lezione" mi ha particolarmente toccato come del resto ha coinvolto tutti i presenti, e non solo emotivamente. Sono momenti gratificanti che ci compensano ampiamente al di là di ogni merito» ${ }^{42}$. Inoltre, il volume è illustrato dallo stesso Bobbio il 10 marzo dello stesso anno a Torino, al Centro Gobetti, per l'apertura del XXIII anno di studi ${ }^{43}$.

Un'occasione importante in questa piccola grande storia della fortuna di Trentin e un impulso al prosieguo del piano di pubblicazione delle Opere scelte è il Centenario della nascita (San Donà di Piave, 11 novembre 1895). La proposta di un convegno per il Centenario viene formulata il 16 marzo 1984 all'assemblea del CEDEI, a Parigi, presso l'Institut Culturel Italien, convocata con il seguente ordine del giorno: 1. I primi anni di attività del CEDEI; 2. «Le colloque Trentin»; 3. I rapporti tra il CEDEI e le istituzioni aderenti; 4. Organizzazione e finanze; 5. Varie ed eventuali ${ }^{44}$.

${ }^{40}$ Lettera dattiloscritta su carta intestata «Biblioteca comunale di Jesolo - Centro di ricerca "Silvio Trentin" per la documentazione e lo studio dell'antifascismo e la Resistenza nel Veneto", Jesolo, 16 maggio 1979 (ANB 1272).

${ }^{41}$ Marsilio, Venezia 1983. Vedi: G. Spadolini, La legge dell'esule. L'avventura di un libro perduto, «La Stampa», XVIII (189), venerdì 10 agosto 1984 e con il titolo Trentin: le leggi severe della democrazia, «La Voce Repubblicana», venerdì 10-sabato 11 agosto 1984, p. 3 (ANB 1272). Secondo Pizzorusso l'opera presenta "alcuni elementi di debolezza», ma ritiene che, "quand'anche si dovesse ammettere che il libro non presenti motivi di interesse tali da renderlo straordinariamente attuale", "toglierlo dall'oblio in cui è rimasto sinora sarebbe comunque doveroso, se non altro per colmare la lacuna derivante dalla mancanza di studi sul fascismo condotti da contemporanei non fascisti». Lettera dattiloscritta su carta intestata "Università degli Studi di Pisa, Istituto di diritto pubblico", datata Pisa, 3 novembre 1980 (ANB 1272).

${ }^{42}$ Lettera autografa su carta intestata, 27 gennaio 1984 (ANB 3872).

${ }^{43}$ Il manoscritto (11 pagine, conservato in ANB 1272) viene utilizzato da Bobbio per preparare una ampia nota Silvio Trentin e lo Stato fascista, pubblicata in «Belfagor», XL (6), 30 novembre 1985, pp. 700-707, ora compresa in questo volume.

${ }^{44}$ Si veda lettera circolare del Presidente del CEDEI, Pierre Milza, Parigi, 14 febbraio 1984 (ANB 380). 
Bobbio non partecipa a questa riunione. Le decisioni gli vengono comunicate da Alberto Cabella con una lettera del 2 aprile da Parigi. L'organizzazione viene affidata a Aldo Vitale e allo stesso Cabella, che considera la scelta di occuparsi di Trentin estremamente significativa, in quanto egli «è forse l'unico antifascista che si sia integrato nella società francese» al punto da diventarne «un protagonista della vita politica e culturale». I primi contatti sono con Robert Paris, interessato «a investigare sui meandri soreliani e sui liberali di sinistra». Inoltre, Cabella suggerisce che Rosengarten si occupi delle numerose opere di Trentin pubblicate in lingua francese e manifesta delle perplessità sulla centralità da assegnare nella struttura del convegno al libro Dallo statuto albertino al regime fascista, curato da Pizzorusso. Un contributo importante viene dato da Carla Gobetti con la ricerca documentaria e i contatti tra i relatori e gli organizzatori ${ }^{45}$.

Bobbio viene coinvolto nel progetto del convegno per il centenario della nascita di Trentin fin dall'inizio come risulta da un altro scambio con Rosengarten, che il 9 marzo 1984, gli manifesta l'intenzione e il desiderio di dare un contributo al colloquio parigino ${ }^{46}$. Rinnovando $i$ sentimenti di "viva ammirazione e amicizia» per Rosengarten, con una lettera del 10 aprile Bobbio lo informa che «il convegno ha soprattutto lo scopo di ricordare i rapporti di Trentin con la Francia» e che quindi le relazioni devono concernere in particolar modo la sua «attività francese». Con il suo consueto pessimismo aggiunge che «i tempi sono molto tristi e si ha l'impressione di aggirarsi nel vuoto ${ }^{47}$. In una lettera successiva, il 10 giugno, lo invita a "tratteggiare un ritratto generale» di Trentin ${ }^{48}$. Il 18 giugno Rosengarten propone un profilo "politico-morale» e anche "umano e privato", con particolare riguardo all'esperienza francese, suggerendo il titolo: Un uomo e una nazione ${ }^{49}$. Proposta accolta con interesse da Bobbio il 7 luglio: da questa lettera traggo una sua considerazione di carattere generale: "L'Italia è un paese logorante, dove uno studioso non si può chiudere nel suo "particulare", anche se poi le sue sortite fuori dall'accademia non servono assolutamente a nulla»"

${ }^{45}$ Lettera dattiloscritta su carta intestata: «C.E.D.E.I. Centre d'Études et de Documentation sur l'Émigration Italienne - c/o Institut Culturel Italien - 50, Rue de Varenne, 75007 Paris», datata Parigi, 2 aprile 1984 (ANB 380). Ad Alberto Cabella, amico, gobettiano e studioso di Gobetti, nonché della Riforma e dell'europeismo è dedicato il volume Piero Gobetti e la Riforma in Italia, a cura di M. Vicari, prefazione di V. Spini, aras edizioni, Fano (PU) 2018; in particolare: P. Polito, Alberto Cabella gobettiano, pp. 35-57 e A. Panero, L'europeismo di Alberto Cabella, pp. 59-63.

46 Lettera dattiloscritta su carta non intestata, 9 marzo 1984 (ANB 380). 380).

47 Lettera dattiloscritta su carta non intestata, Torino, 10 aprile 1984, in copia (ANB

${ }^{48}$ Lettera dattiloscritta su carta non intestata, Torino, 10 giugno 1984 (ANB 380).

${ }^{49}$ Lettera dattiloscritta su carta non intestata, 18 giugno 1984 (ANB 380).

${ }^{50}$ Lettera dattiloscritta su carta non intestata, 7 luglio 1984, in copia (ANB 380). 
Il 5 dicembre 1984 Bobbio scrive al Presidente della Repubblica Sandro Pertini, chiedendo di essere ricevuto per invitarlo a intervenire, l'8 febbraio 1985, alla seduta augurale del Convegno su Silvio Trentin e la Francia ${ }^{51}$. L'incontro è avvenuto prima delle vacanze di Natale con la partecipazione di Carla Gobetti ${ }^{52}$. Il Presidente non poté partecipare ai lavori, ma nel messaggio inviato al convegno pone l'accento sull'azione di Trentin che «fu costantemente rivolta a salvaguardare l'intesa e la collaborazione tra tutte le forze", aggiungendo: "Così io lo ricordo e così lo conobbero i compagni d'esilio e gli amici francesi, che ben presto appresero ad amarne le qualità umane e a stimarne la profondità di pensiero, il personale disinteresse, la totale dedizione ${ }^{53}$.

Il convegno per il Centenario si tiene a Parigi, l' 8 e 9 febbraio $1985^{54}$. È l'occasione per approfondire il pensiero sul federalismo di Trentin. Bobbio presenta nella sezione «La pensée politique de Trentin» la relazione La pensée fédéraliste de Trentin. Gli atti del convegno sono pubblicati con qualche ritardo nel volume Silvio Trentin e la Francia. Saggi e testimonianze, prefazione di Giannatonio Paladini, Marsilio, Venezia 1991. Nell'introduzione Bobbio osserva che i vari contributi favoriscono «un allargamento e un approfondimento della conoscenza di un'opera che non esitiamo a considerare una delle più rappresentative e ancor oggi ricche di riflessioni attuali, prodotte dalla diaspora antifascista, democratica e socialista ${ }^{55}$. In una lettera a Zannoner del 4 febbraio 1991, dopo avere scritto: «abbiamo adempiuto al nostro impegno con gli amici francesi», rende omaggio all'allora direttore della Maison de l'Italie Aldo Vitale, venuto a mancare «nella pienezza delle sue forze e della sua intelligenza» e commenta: «Il libro mi pare riuscito bene. Il contenuto è vario e interessante. Un degno omaggio a quel degno uomo che fu Silvio Trentin» ${ }^{56}$.

${ }^{51}$ Lettera su carta intestata «Senato della Repubblica», Torino, 5 dicembre 1984 (ANB 380).

52 Il 18 luglio 1984 Bobbio venne nominato senatore a vita «per altissimi meriti nel campo sociale scientifico, artistico e letterario» dal presidente della Repubblica Sandro Pertini. Nella lettera dianzi citata scrive tra l'altro: «[...] gradirei farti gli auguri personalmente e mi piacerebbe parlarti delle mie prime esperienze di parlamentare (apprendista)». (ANB 380).

${ }^{53}$ Si veda la documentazione conservata nel fascicolo ANB 380.

${ }^{54}$ I lavori di Parigi hanno avuto una certa eco sui giornali italiani: G. Paladini, Trentin e la "scuola morale della trincea», "Il Gazzettino", 10 gennaio 1985; L. Bocchi, Un omaggio a Trentin emigrato antifascista, "Corriere della sera", 20 gennaio; L. Ferraro, Silvio Trentin e la Francia. Riscoperta di un antifascista, "La Nuova Venezia», 8 febbraio; N. Bobbio, Silvio Trentin, la Francia e quell'idea dell'Europa, «La Stampa», 9 febbraio; S. Lanaro, Fu un liberale antifascista che scelse la classe operaia, "La Nuova Venezia», 10 febbraio; M. Revelli, Silvio Trentin scomodo e attuale, «Il Manifesto», 15 febbraio. La notizia apparve nella pagina culturale di «Le Monde», 6 febbraio (Cfr. ANB 380).

55 Silvio Trentin e la Francia. Saggi e testimonianze, prefazione di G. Paladini, Marsilio, Venezia 1991, p. 18.

${ }^{56}$ Lettera manoscritta su carta intestata «Senato della Repubblica», Torino, 4 febbraio 1991 (ANB 1272). 
5. Avviandoci alla conclusione, torniamo al progetto di edizione delle Opere scelte. Al già ricordato volume Dallo Statuto albertino al regime fascista, a cura di Alessandro Pizzorusso, seguono nel 1984 Politica e amministrazione. Scritti e discorsi 1919-1926, a cura di Moreno Guerrato ${ }^{57}$; nel 1985 Antifascismo e rivoluzione. Scritti e discorsi 1927-1944, a cura di Giannantonio Paladini ${ }^{58}$; nel 1987 Federalismo e libertà. Scritti teorici 1935-1943, a cura di Bobbio che vi pubblica come introduzione, riveduta e annotata, la relazione presentata al Convegno del Centenario ${ }^{59}$; nel 1988 Diritto e democrazia. Scritti sul fascismo 1928-1937, ancora a cura di Angelo Ventura ${ }^{60}$.

Il progetto prevedeva l'edizione dell'Epistolario, a cura di Rosengarten, che non è stata realizzata ${ }^{61}$, il reprint di Libérer et Fédérer ${ }^{62} \mathrm{e}$ la traduzione completa e parzialmente corretta di La Crise du Droit et de l'État, a cura di Franca Trentin. Come si è già detto, fin dal giugno 1975 la pubblicazione del libro è uno degli argomenti principali nel carteggio tra Rosengarten e Zannoner. Si è già ricordato il parere negativo espresso da Bobbio nelle lettere a Federico Codignola e a Raffaello Zannoner (22 giugno e 8 luglio 1976). Diversamente Rosengarten ha una opinione opposta. Lo studioso americano in una lettera del 16 febbraio 1979 scrive a Zannoner di non condividere «l'opinione di Bobbio sulla vecchiaia di La Crise du Droit et de l'État ${ }^{63}$. L'atteggiamento di Bobbio è stato certamente determinante

${ }^{57}$ Marsilio, Venezia 1984.

${ }_{58}$ Marsilio, Venezia 1985.

${ }_{59}$ Marsilio, Venezia 1987, pp. IX-XXXVII, ora in questo volume. Bobbio invia a Raffaello Zannoner la prima copia della sua introduzione. Cfr. lettera dattiloscritta su carta non intestata, datata Torino, 13 settembre 1985, in copia (ANB 1273). Il volume viene presentato dallo stesso Bobbio a Venezia il 6 dicembre 1986. Il testo manoscritto del discorso è conservato in ANB 1273 e si può leggere ora in questo volume.

${ }^{60}$ Marsilio, Venezia 1988. Tra i due esiste una consonanza ideale e civile «in difesa di questa nostra Repubblica e della sua costituzione, che se necessita di alcune rettifiche non è poi così inadeguata e superata dalla storia, come vorrebbero i fautori di un certo torbido presidenzialismo plebiscitario, insofferente degli strumenti della democrazia rappresentativa e del principio di legalità». Lettera dattiloscritta di A.Ventura a N. Bobbio su carta intestata «Università degli Studi di Padova», datata Padova, 31 maggio 1991 (ANB 3780).

${ }^{61}$ Sui carteggi sta lavorando con profitto Carlo Verri: Id., Caro Nenni. Appunti per un epistolario di Silvio Trentin, "Annali della Fondazione Ugo La Malfa», 24, 2009, pp. 149169; Id., Il primo antifascismo in esilio in una lettera di Trentin a Turati, «Italia Contemporanea», 252-253, 2008, pp. 529-538; Id., Trentin-Grieco. Un carteggio nel mezzo della guerra d'Etiopia, «Italia Contemporanea», 242, 2006, pp. 95-120. A cura dello stesso, è in progetto la pubblicazione del carteggio di Silvio e Beppa Trentin con Luigi Luzzatti.

${ }^{62}$ Libérer et Fédérer: 14 juillet 1942-avril-mai 1944, présentation de Michel Dreyfus, publié avec le concours des municipalités deVenise et de Jesolo et du Centro di ricerca Silvio Trentin.

${ }^{63}$ Il carteggio tra Rosengarten e Zannoner è conservato al Centro Silvio Trentin di Jesolo. Sulla vicenda della pubblicazione de La Crise sono da vedere le considerazioni svolte da Gangemi, Bobbio e Trentin tra Resistenza e filosofia del diritto, cit., pp. 62-75. L'autore sostiene che "possiamo ipotizzare due diversi e contrastanti criteri di valutazione dell'opera di Trentin: coloro che hanno un interesse scientifico [...]; coloro che hanno un interesse eminentemente storico". Per l'influenza di Bobbio negli studi trentiniani, 
nella scelta di editare La crise solo parzialmente. Infatti, viene adottata la decisione di pubblicarne solo le conclusioni nel volume curato da Bobbio Federalismo e libertà. Scritti teorici $1935-1943^{64}$, mentre nel volume curato da Ventura, Diritto e democrazia. Scritti sul fascismo 1928-1937 viene inserito il capitolo VII, dedicato al rapporto tra capitalismo e fascismo ${ }^{65}$.

Successivamente La crise è stata tradotta e pubblicata da Giuseppe Gangemi. In una lettera a Gangemi dell'11 luglio 2004 Franca Trentin scrive: «rimane nella mia mente un po' misteriosa questa passione insistente per l'opera di mio padre, ma è anche una cosa lusinghiera» ${ }^{66}$. Secondo Gangemi, nella ricca produzione storica, giuridica e politica di Trentin La crise è «la sua opera maggiore», il suo «volume più importante: un libro che gli è costato molto in termini di fatica intellettuale e anche di risorse economiche da investire», un'opera "che fu fortemente meditata e che fu il risultato di un confronto con tutta la letteratura filosofica e giuridica (italiana, francese, tedesca e di lingua inglese) più importante del tempo" ${ }^{67}$.

Bobbio riconsidererà almeno in parte il giudizio su La crise nell'ultimo scritto dedicato a Trentin, intitolato François Gény: una prefazione (che Gangemi non cita nella sua introduzione alla edizione italiana) dove il filosofo scrive:

La crisi del diritto e dello stato è dei libri di Trentin dell'esilio il più ambizioso e teoricamente importante, ma è anche quello meno fortunato. [...] Nell'evoluzione del pensiero di Trentin quest'opera è importante, perché è quella in cui appare per la prima volta proposta con determinazione l'idea del federalismo come soluzione della crisi del diritto e dello stato, conseguenza dello strapotere politico ed economico quale si è venuto formando in una società capitalistica, soffocante sempre più l'autonomia dei gruppi e calpestante la dignità della persona ${ }^{68}$.

ha finito per «prevalere la valutazione storica» (pp. 74-75), lasciando in ombra il giurista, che, secondo Gangemi, soprattutto nell'opera La Crise mostra la sua attualità e vitalità.

${ }^{64}$ Ivi, pp. 3-33.

${ }^{65}$ Ivi, pp. 219-228.

${ }^{66}$ Lettera su carta intestata: «Franca Trentin Baratto», Venezia, 11 luglio 2004. Si veda la lettera su carta intestata: «Università di Padova. Dipartimento di studi storici», Padova, 18 giugno 2004, con la quale Gangemi richiede l'autorizzazione a tradurre e a pubblicare il volume e quella successiva del 30 giugno 2005, con la quale informa i familiari degli sviluppi del lavoro. Le lettere sono conservate presso l'Archivio Franca Trentin - Casa della Memoria, Associazione Resistente, Venezia.

${ }^{67}$ Cito dall'ampio saggio introduttivo del curatore, Silvio Trentin, il diritto naturale e la libertà come autonomia, pp. 7 e 10-11, in S. Trentin, La Crisi del Diritto e dello Stato, prima ed. italiana, a cura di G. Gangemi, Gangemi Edizioni, Roma s.d. [ma: 2006]. Segnalo che il libro era stato tradotto da Livio Sovelli, Silvio Trentin, La Crise du Droit et de l'État, Università Ca' Foscari di Venezia, Facoltà di Lingue e Letterature straniere, a.a.19791980. La tesi è depositata presso il Fondo Trentin di Jesolo.

68 «Quaderni per la storia del pensiero giuridico moderno", 20, 1991, pp. 181-187 (ANB 1274), ora in questo volume. 
Per completare il quadro delle iniziative editoriali trentiniane occorre ricordare la pubblicazione del saggio manoscritto inedito Le determinanti dialettiche e gli sbocchi ideologici e istituzionali della rivoluzione antifascista [europea], conservato nel Fondo Silvio Trentin del Centro studi Piero Gobetti ${ }^{69}$. La stesura risalente al marzo 1944 consente di «arguire che esso rappresenta con tutta probabilità l'ultima fatica di Trentin, troncata dalla morte: una riflessione lucida e appassionata della storia novecentesca, sulla crisi della società contemporanea. Un documento che potremmo definire, a posteriori, una sorta di testamento politico» ${ }^{70}$.

Nella prefazione Marco Revelli opportunamente richiama le assonanze e le convergenze tra Piero Gobetti e Silvio Trentin. In primo luogo l'esilio: entrambi furono costretti a lasciare l'Italia ed entrambi scelsero la Francia, Trentin il 2 febbraio 1926, Gobetti il 3 febbraio dello stesso anno. In secondo luogo il valore dell'autonomia, «l'elemento che rese rivoluzionario il loro liberalismo». In terzo luogo la medesima visione dell'impegno: "una concezione attiva, militante, della cultura mai separata dall'azione. E l'attribuzione all' "intellettuale" di una responsabilità forte, impegnativa, come chi è chiamato, appunto, a testimoniare di persona dei propri valori e dei propri concetti ${ }^{71}$.

7. Concludo questa ricostruzione della riproposizione e diffusione dell'opera di Trentin con lo scambio epistolare avvenuto tra Bobbio e Rosengarten all'inizio del 1998. L'occasione è la lettura da parte dell'americano del De senectute di Bobbio, uscito da Einaudi nel 1996. In una lettera del 19 gennaio 1998, Rosengarten ricapitola per l'amico le vicende private e pubbliche della sua vita, ricordando il lavoro per l'introduzione di Gramsci negli Stati Uniti e anche la triste perdita del figlio maggiore, Philip, all'età di 36 anni. Il suo commento al De Senectute è di grande interesse. Egli apprezza «quel certo sale stilistico e quel certo umorismo ironico» che gli sembrano "tratti inconfondibili del tuo carattere, in persona e anche nel tuo stile» che emerge quando Bobbio si occupa di «casi e cose personali e non di problemi giuridici». Augurando al De senectute di diventare un classico tra le opere di filosofia morale, dichiara di non essere d'accordo su un punto:

69 S. Trentin, Le determinanti dialettiche e gli sbocchi ideologici e istituzionali della rivoluzione antifascista [europea]. Saggio inedito del 1944, a cura e con una introduzione di C. Malandrino e una prefazione di M. Revelli, Lacaita, Manduria-Bari-Roma 2007.

${ }^{70}$ C. Malandrino, Introduzione, in Trentin, Le determinanti dialettiche e gli sbocchi ideologici e istituzionali della rivoluzione antifascista [europea]. Saggio inedito del 1944, cit., p. 13.

71 M. Revelli, Prefazione, ivi, p. 9. "Il Centro studi Piero Gobetti è orgoglioso di ospitarne la pubblicazione, nella certezza di compiere non solo un'opera doverosa di completamento della bibliografia trentiniana, ma anche di consegnare al pubblico una testimonianza preziosa di quell'“altra Italia" che seppe pensarsi diversa in un'epoca in cui tutto sembrava perduto, e per questo poté preparare un "futuro civile"” (ivi, p. 10). 
L'unica cosa che mi ha un po' arruffato è l'associazione che fai fra vecchiaia e rinunzia ad idee e a posizioni militanti. È certamente vero che gli anni portano con loro un maggiore distacco dalle cose che, da giovani, ci riempivano di sdegno e di angoscia, ma non vedo perché adottare un atteggiamento stoico e spassionato di fronte a tutte le situazioni di ingiustizia ${ }^{72}$.

Nella risposta (12 marzo 1998) Bobbio confida all'amico di essere uscito dall'arena e di essere salito sugli spalti:

Mi sono tirato in disparte non solo per stanchezza, ma anche perché ritengo di non avere più molte cose interessanti da dire, soprattutto ai giovani. Ho sempre avuto un grande senso dei miei limiti. Ora, ce l'ho più che mai. La mia testa diventa sempre più debole, il mondo, al contrario, diventa sempre più complicato. Non credo di avere capito molto in tutti gli anni in cui stavo nell'arena. Adesso che sono salito sugli spalti ho l'impressione di capirne ancora meno. Si aprono attraverso quello che si suole chiamare il mondo digitale, orizzonti sconfinati, di cui non so prevedere i possibili effetti ${ }^{73}$.

Il vecchio filosofo ritrova il senso di un impegno durato una vita nella fedeltà agli stessi ideali di giustizia e di libertà che hanno ispirato l'opera dell'amico:

Credo di essere rimasto fedele, questo sì, ad alcuni valori fondamentali che mi hanno ispirato, anche se sono stato perlopiù impari al compito, nel trapasso dalla teoria, che appartiene alla competenza specifica degli intellettuali, alla pratica, in cui i politici sono molto più bravi, e ce la fanno da padroni ${ }^{74}$.

72 Lettera dattiloscritta su carta intestata, 19 gennaio 1998 (ANB 3390)

${ }^{73}$ La copia della risposta di Bobbio, su carta non intestata, Torino, 12 marzo 1998, si trova in ANB 3390.

${ }^{74}$ Ibidem. 



\title{
NOTA AI TESTI
}

\author{
Pina Impagliazzo, Pietro Polito
}

Di seguito si forniscono le indicazioni bibliografiche dell'itinerario completo degli studi che Norberto Bobbio ha dedicato a Silvio Trentin, per poi indicare alla fine $i$ testi compresi in questo volume, nell'edizione che di essi è stata adottata.

II primo lavoro bobbiano su Trentin è la commemorazione (Venezia 14 marzo 1954) per il decimo anniversario della morte: Silvio Trentin, «Il Ponte», X (5), maggio 1954, pp. 702-713; rist. con il titolo Ricordo di Silvio Trentin, Stampa Arti Grafiche Sosteni, Venezia 1955 e in Id., Italia civile. Ritratti e testimonianze, Manduria-Bari-Perugia 1964, pp. 271-288 (nuova ed. Passigli, Firenze 1986, pp. 249-266, da cui si cita), poi con il titolo Un protagonista dell'Italia civile, in G. Spadolini (a cura di), Silvio Trentin nell'emigrazione antifascista, «Nuova Antologia», 2152, ottobre-dicembre 1984, pp. 22-45.

A Trentin Bobbio dedica alcune pagine nel Profilo ideologico del Novecento (1969), Garzanti, Milano 1990, pp. 189-191.

Inoltre è autore della presentazione di S. Trentin, Scritti inediti. Testimonianze e studi, a cura di P. Gobetti, Guanda, Parma 1972, pp. IX-XII.

Bobbio torna su Trentin in occasione del trentesimo anniversario della morte (San Donà del Piave, 9 novembre 1974), con una relazione pubblicata col titolo La crisi europea e lo stato federale nell'opera di Silvio Trentin, «Città e Regione», I (8), ottobre 1975, pp. 200-212; poi compresa in Atti del Convegno di studio su Silvio Trentin, Jesolo, 20 aprile 1975, Neri Pozza, Vicenza 1976, pp. 109-123.

Sono da vedere i riferimenti che egli fa a Trentin nel suo saggio Il federalismo nel dibattito politico e culturale della Resistenza, in S. Pistone (a cura di), L'idea dell'unificazione europea dalla Prima alla Seconda guerra mondiale, Fondazione Luigi Einaudi, Torino 1975, pp. 234-235.

Con una ampia nota Silvio Trentin e lo stato fascista, «Belfagor», XL (6), 30 novembre 1985, pp. 700-707, si occupa di S. Trentin, Dallo statuto albertino al regime fascista, a cura e con introduzione di A. Pizzorusso, "Opere scelte di Silvio Trentin", Marsilio, Venezia 1983.

Nel 1985 partecipa al convegno Silvio Trentin e la Francia (Parigi, 8 e 9 febbraio), dove presenta una relazione su Il pensiero federalista di Silvio 
Trentin, che costituisce l'Introduzione del volume da lui curato S. Trentin, Federalismo e libertà. Scritti teorici 1935-1943, Marsilio, Venezia 1987, pp. IX-XXXVII. Questo testo è stato raccolto in Silvio Trentin e la Francia. Saggi e testimonianze, prefazione di G. Paladini, Marsilio, Venezia 1991, pp. 147-171, di cui Bobbio è anche autore dell'Introduzione sulle ragioni del colloquio, pp. 15-18.

Infine, Bobbio è autore dello scritto François Gény: una prefazione, "Quaderni per la storia del pensiero giuridico moderno», 20, 1991, pp. 181-187.

I testi compresi nel volume vengono proposti nella forma originaria, senza interventi redazionali di carattere storico, linguistico e nemmeno bibliografico. Per non appesantire inutilmente la lettura, si è preferito rinviare all'ampia bibliografia posta in appendice. I rari casi in cui i curatori sono intervenuti con integrazioni e informazioni nelle note vengono indicati tra parentesi quadre.

1. Silvio Trentin, in Italia civile. Ritratti e testimonianze, Manduria-Bari-Perugia 1964, pp. 271-288; nuova ed. Passigli, Firenze 1986, pp. 249266, con una nuova prefazione con data: «Torino 25 aprile 1985». La prima edizione del libro uscì senza una dedica. La seconda è dedicata a Franco Antonicelli. In appendice: Aggiornamenti bibliografici, a cura di P. Polito.

2. La crisi europea e lo stato federale nell'opera di Silvio Trentin, in Atti del Convegno di studio su Silvio Trentin, Jesolo, 20 aprile 1975, Neri Pozza, Vicenza 1976, pp. 109-123.

3. Silvio Trentin e lo stato fascista, «Belfagor», XL (6), 30 novembre 1985, pp. 700-707.

4. L'opera di Silvio Trentin, discorso pronunciato a Venezia il 6 dicembre 1986, in occasione della presentazione del IV volume delle "Opere scelte di Silvio Trentin", Federalismo e libertà. Scritti teorici 1935-1943, Marsilio, Venezia 1987, inedito.

5. Introduzione a S. Trentin, Federalismo e libertà. Scritti teorici 1935-1943, Marsilio, Venezia 1987, pp. IX-XXXVII.

6. François Gény: una prefazione, "Quaderni per la storia del pensiero giuridico moderno», 20, 1991, pp. 181-187.

In appendice sono pubblicate le seguenti lettere:

Agostino Zanon Dal Bo, Venezia, 18 febbraio 1954;

Beppa Nardari Trentin, [dicembre 1953];

Beppa Nardari Trentin, senza data [precedente al 14 marzo 1954];

Bruno Trentin, senza data [precedente al 14 marzo 1954];

Aldo Garosci, Roma, 3 marzo [1954];

Beppa Nardari Trentin, senza data [successiva al 14 marzo 1954];

Norberto Bobbio, Torino, 28 marzo 1954. 
TESTI 

In una conferenza su Giacomo Leopardi, tenuta a Tolosa il 13 gennaio 1940 (la guerra mondiale si era scatenata da alcuni mesi) Silvio Trentin scriveva: "A noi che il lungo esilio ha consumato in tormenti senza nome, accade di ricavare dal suo pessimismo grande conforto. È il poeta della disperazione che ci insegna meglio d'ogni altro a non disperare. È questo implacabile denunciatore delle miserie che rendono così vile la condizione umana, che ci dà la possibilità di comprendere appieno la necessità improrogabile e l'incomparabile bellezza della lotta in cui abbiamo impegnato la nostra vita»'.

Lo stato d'animo dell'esiliato era diviso tra la nostalgia della patria lontana, che lo aveva indotto a cercar conforto nel poeta del dolore, e la speranza che sofferenze, lotte, sciagure non sarebbero state inutili al fine di riconquistare, insieme con la patria perduta, nuove forme di giustizia e di fraternità tra i popoli.

Alcuni anni prima, nella premessa ad un'opera di teoria politica, affermando che l'esilio «è ben lungi dall'essere soltanto miseria o rancore o insensata e caparbia presunzione» citava un passo di Victor Hugo, nel quale evidentemente aveva ritrovato il proprio migliore ritratto:

«Sognare, pensare, soffrire. Essere solo e sentir che si e nello stesso tempo solidale con tutti e con ognuno... Maturare la propria coscienza civica e raffinare le proprie speculazioni filosofiche. Essere povero e far fronte alla propria rovina col proprio lavoro. Meditare e premeditare: meditare il bene e premeditare il meglio. Non conoscere altra collera se non quella che s'ispira e s'infiamma alla preoccupazione disinteressata del pubblico bene. Ignorare l'odio personale. Respirare l'aria vivificatrice delle solitudini infinite... Non spingere mai la contemplazione dell'ideale sino a dimenticare l'esistenza del tiranno. Ritrovare in se stesso, magnificamente confuse, l'indignazione che non cessa di rafforzare e d'in-

* Silvio Trentin, in Italia civile. Ritratti e testimonianze, Manduria-Bari-Perugia 1964, pp. 271-288; nuova ed. Passigli, ivi, Firenze 1986, pp. 249-266. pp. 118 .

Norberto Bobbio, L'esempio di Silvio Trentin. Scritti 1954-1991, edited by Pina Impagliazzo, Pietro Polito, ( 2020 Author(s), content CC BY 4.0 International, metadata CC0 1.0 Universal, published by Firenze University Press (www.fupress.com), ISSN 2612-7946 (online), ISBN 978-88-5518-018-4 (online PDF), ISBN 978-88-5518-681-0 (XML), 
grandire e la calma serena che diviene ognor più inaccessibile al dubbio. Avere due anime: la propria e quella della patria lontana». Anche Silvio Trentin era diventato povero e aveva dovuto far fronte alla propria rovina col proprio lavoro. Aveva conosciuto l'amarezza della solitudine e la gioia della solidarietà con gli oppressi di tutti i paesi che si preparavano a risorgere, e di questi s'era fatto in ogni occasione esortatore e collaboratore, amico e compagno. Aveva dedicato quegli anni con intensissimo studio a meditare il bene e a premeditare quel migliore assetto politico che sarebbe dovuto sorgere dall'incendio delle vecchie strutture. Non aveva conosciuto l'odio personale per gli avversari, ma solo l'indignazione che accende l'uomo civile di fronte all'applaudito ritorno del barbaro. E l'indignazione era pur moderata in lui dalla serena certezza di avere scelto la giusta parte. Nella sua anima, insomma, rivolta in quegli anni continuamente a riflettere sui problemi italiani del presente e del futuro, non aveva dimenticato, anzi aveva meglio ritrovato, l'anima della patria.

A questa lunga meditazione degli anni dell'esilio, egli era pervenuto con una eccezionale esperienza congiunta di giurista e di uomo politico: rara esperienza in Italia dove, com'è noto, l'orientamento della scienza giuridica è prevalentemente tecnico e orgoglioso di essere tale, e i politici fanno del loro meglio, manipolando il diritto a lor piacimento, per incoraggiare i puri tecnici nella sopravvalutazione della loro opera.

Nato a S. Donà di Piave nel novembre del 1885, laureatosi in giurisprudenza a Pisa, si era dedicato agli studi di diritto pubblico sotto la guida di Giovanni Vacchelli, conseguendo nel 1910 la libera docenza in diritto amministrativo, e quindi professando tale materia nel 1910-11 alla libera Università di Camerino. Due opere di mole e su temi d'impegno, l'una sulla Responsabilità collegiale, l'altra sulla Cosa giudicata nelle decisioni delle sezioni giurisdizionali del Consiglio di Stato, apparse entrambe nel 1910, quando egli aveva appena 25 anni, lo avevano messo in luce tra i giovani promettenti cultori di un ramo delle scienze giuridiche che si avviava in quegli anni ad una elaborazione tecnicamente più rigorosa, ad una revisione e sistemazione dei suoi concetti generali. Nel 1914, dopo un periodo di permanenza ad Heidelberg, quale lettore in quella Università, ove aveva potuto profittare dell'insegnamento di un insigne giurista, Fritz Fleiner, pubblicò un'opera più matura sull'Atto amministrativo, vera e propria trattazione di teoria generale del diritto pubblico, in cui nella scia della scienza giuridica tedesca tecnicamente più avanzata, si affrontavano i principali problemi dogmatici e sistematici del diritto pubblico, che richiedevano soluzioni appropriate, distinte dalle comode e affrettate trasposizioni di concetti tratti dal diritto privato.

Il libro era stato scritto alle soglie dello scoppio del primo conflitto mondiale. Due anni dopo l'autore, poco più che trentenne, era ufficiale aviatore volontario, e meritava tre croci di guerra al valore. La guerra non rappresentò soltanto un temporaneo distacco dagli studi, trascorso il qua- 
le avrebbe ripreso le occupazioni abituali, ma fu per il Trentin, come per tanti intellettuali italiani, una presa di coscienza di nuovi valori umani, un approfondimento della posizione e della responsabilità dell'uomo di cultura nella vita sociale, una comprensione più matura, più viva, degli insoluti problemi nazionali. Tornava comodo a coloro che nella guerra non avevano visto che l'avventura a lieto fine o volevano da essa trarre profitto, stordirsi nella retorica patriottica. Trentin vedeva nella guerra una terribile prova da cui erano emerse, con un aspetto inquietante, antiche e croniche insufficienze della struttura sociale ed economica italiana.

Nel 1919 scriveva che chi avesse il coraggio di guardare in faccia la realtà, doveva riconoscere che vi erano immensi problemi da affrontare: «La verità è venuta a galla: la nostra formale indipendenza manca di vero e sostanziale contenuto. Il funzionamento della nostra vita collettiva è assolutamente precario; tutte le nostre forze ed ogni nostro organismo mancano dei mezzi indispensabili per la loro espansione... La povertà c'insidia, nonostante ogni nostra rinunzia, persino nell'esistenza materiale subordinando ai capricci dello straniero lo stesso approvvigionamento alimentare ${ }^{2}$. Era sua opinione che la guerra ci avesse fatto acquistare l'indipendenza politica, ma che gl'italiani dovessero d'ora innanzi por mano alla loro indipendenza economica. A quest'opera urgente di pace, infatti, egli si dedicò entrando nella vita politica. Nelle prime elezioni del dopoguerra, nel 1919, fu eletto deputato quale candidato nella lista della democrazia sociale.

La sua competenza di giurista, esperto in quella sfera del diritto che riguarda la pubblica amministrazione, lo distingueva sia dai politici generici sia dagli astratti ideologi. D'altra parte la sua probità morale non lo faceva confondere con gl'intriganti, grandi o piccoli, con le schiene curve o pronte a curvarsi che avrebbero accettato di lì a pochi anni un padrone armato di bastone. Era un uomo politico nuovo, emerso dalla prima competizione elettorale a suffragio veramente universale: il politico che è insieme uomo di studi e di retta coscienza, l'intellettuale che sente i problemi della vita civile come problemi non solo teorici ma di pratica organizzazione. Convinto che il primo problema da affrontare fosse il problema economico, e che la terra fosse la principale fonte di ricchezza del paese, spiegò la sua attività di pubblico amministratore soprattutto nelle opere di bonifica. Creò l'Ente di rinascita agraria per le province di Venezia e di Treviso, che si estese poi a tutte le province del Veneto. $\mathrm{Fu}$, credo, uno dei suoi maggiori vanti, se devo giudicare dall'accenno, inaspettato, che si legge in una delle sue opere principali dell'esilio, là dove, difendendo l'economia collettivistica, adduce l'esempio - con qual

${ }^{2}$ Per un nuovo orientamento della legislazione in materia di bonifiche in rapporto alle presenti esigenze dell'economia nazionale, Tip. della "Cooperativa del popolo", Venezia 1919, p. 4. 
melanconico sguardo sul passato si può ben immaginare - di questo ente per provar la piena idoneità della pubblica impresa ad adempiere con successo al compito di una gestione modello ${ }^{3}$.

Intanto i suoi studi erano diventati più aderenti ai problemi concreti; non più opere di dogmatica o di teoria generale del diritto pubblico, ma di riforma legislativa e amministrativa. La tecnica del giurista ch'egli aveva così bene appresa diventò appropriato strumento per il conseguimento di fini sociali. Scrisse nel 1919 un saggio Per un nuovo orientamento della legislazione in materia di bonifiche in rapporto alle presenti esigenze dell'economia nazionale, nel quale sosteneva la necessità di porre limiti al diritto di proprietà e propugnava l'intervento dello stato nelle opere di bonifica. Attese in special modo ad uno studio particolareggiato e documentato sulle condizioni, sui mezzi e sulle possibilità di una assunzione del servizio pubblico delle utilizzazioni idrotecniche da parte degli enti provinciali del Veneto, mostrando già alcuni principi o tendenze che si sarebbero sviluppate nelle sue future opere di teoria politica, massime la critica della concezione liberistica e privatistica dell'economia, la fiducia nell'opera degli enti locali per l'esercizio di attività economiche di pubblico interesse, e soprattutto nei benefici di una sana amministrazione decentrata. È l'opera più importante di questo periodo, uscita nel 1922 col titolo: Gli enti pubblici del Veneto di fronte al problema delle utilizzazioni idrotecniche ${ }^{4}$. Si appassionò infine ai problemi delle terre redente, e di questo interesse rimane testimonianza nell'opera del 1923: La restaurazione delle terre liberate in Italia ${ }^{5}$.

Dopo aver insegnato a Camerino e a Macerata, era chiamato nel 1923 a insegnare istituzioni di diritto pubblico e diritto internazionale nell'Istituto Superiore di Scienze economiche e commerciali di Venezia.Vi teneva un discorso inaugurale su Autonomia, autarchia, decentramento ${ }^{6}$, in cui sosteneva, pur opponendosi ai federalisti e ai regionalisti, e fondandosi sul concetto giuridico di autarchia, che si dovesse rinnovare su basi di maggiore decentramento l'amministrazione pubblica in Italia, e concludeva con un elogio alla democrazia, pronunciando parole che, in quei giorni, erano un'aperta sfida al nuovo regime: «Perché il tono della vita pubblica si elevi, perché l'ordinamento della pubblica amministrazione possa fruttuosamente concorrere ad un sempre più rapido e perfezionato sviluppo dei rapporti di coesistenza, bisogna che il cittadino non sia abituato ad abdicare davanti alla volontà altrui, a rassegnarsi davanti alla forza: bisogna che non sia mai in lui oscurata la coscienza della sua responsabilità»?.

${ }^{3}$ Riflessioni sulla crisi e sulla rivoluzione, ESIL, Marseille, p. 126.

${ }^{4}$ Officine Grafiche C. Ferrari, Venezia 1922, pp. 391.

5 Zanichelli, Bologna 1923.

${ }^{6}$ Officine Grafiche C. Ferrari, Venezia 1925, pp. 59.

7 Ivi, p. 44. 
Nessuno più di lui aveva diritto di pronunciare queste parole: egli non abdicò dinanzi alla volontà altrui, non si rassegnò davanti alla forza; e della propria responsabilità ebbe sempre la più chiara coscienza. La sua azione politica era mossa da una radicata fiducia nella democrazia: se le aspre difficoltà del dopoguerra avevano scosso le istituzioni tradizionali, non le avevano fatte crollare. La dura prova subita ne aveva se mai dimostrata la solidità. Occorrevano uomini capaci e attivi, programmi precisi di riforma, dirittura di intenzioni e di opere. Ed egli si adoprò in quegli anni, per quanto poté, promovendo le iniziative di cui abbiamo discorso e partecipando ad opere di riforma e ai lavori parlamentari, a rendere effettivo il suo ideale democratico e a infondere la fiducia nella democrazia in pubblici dibattiti. Alle convinzioni democratiche - che costituiscono il tratto più caratteristico e continuo della sua personalità politica - rimarrà fedele tutta la vita, adattandole al mutar dei problemi e delle prospettive, ma non mai affievolendole né alterandole. Passerà dalla democrazia radicale di questi anni ad una forma di democrazia giacobina nell'ultimo periodo dell'esilio. Ma sarà in lui costante la preoccupazione di non venir meno allo spirito della democrazia come governo della libertà. Di ogni forma di antidemocrazia, a cominciare da quella fascista, fu irremovibile avversario. Resistette alla forza da uomo libero e impavido. E quando il regime fascista si affermò, egli fu di quella sparuta minoranza che trasse tutte le conseguenze dagli ideali professati e dall'opera compiuta: preferì l'esilio alla sottomissione. Degli emigrati appartenne alla primissima schiera: partì per la Francia il 2 febbraio del 1926. Fu uno dei tre professori universitari che rinunciarono sdegnosamente alla cattedra, dopo che fu emanata la legge del 24 dicembre 1925, che toglieva agli universitari la prerogativa della inamovibilità nel loro grado e nel loro posto, e che attribuiva al governo la facoltà di revocare coloro che avessero professato idee politiche non ortodosse: gli altri due furono, nobilissima compagnia, F. S. Nitti e G. Salvemini. Accettarono la revoca piuttosto che abdicare al diritto del libero insegnamento, Arturo Labriola ed Enrico Presutti ${ }^{8}$.

Non lo attendevano in Francia né cattedre, né uffici retribuiti ove potesse esplicare la propria attività di studioso. Nei primi anni (1926-29) fece l'agricoltore a Pavie (Guascogna). Poi fu costretto per vivere ad esercitare per alcuni anni il mestiere dell'operaio tipografo ad Auch (1929-34). Per il resto del suo soggiorno francese, condusse una libreria a Tolosa. Ma non rinunciò, pur nelle difficoltà materiali, alla sua missione d'insegnante e ai suoi doveri di studioso. Tenne in quei quindici anni anche la sua

8 Traggo queste notizie dallo stesso Trentin che accenna al suo distacco dall'Università e a quello dei colleghi nell'opera Les transformations récentes du droit public italien, Marcel Giard, Paris, 1949, pp. 439-440. 
cattedra - una cattedra più viva ed alta di quella di tanti colleghi rimasti tranquillamente in patria - attraverso le numerose opere di critica politica e di teoria del diritto e dello stato che andò via via pubblicando. A veder oggi l'elenco dei libri ch'egli scrisse in esilio - di cui alcuni sono rimasti inediti e la maggior parte purtroppo sono pressoché sconosciuti e, come ben si comprende, introvabili nelle nostre biblioteche - ci si domanda con meraviglia com'egli avesse potuto compiere tanto lavoro nelle infelicissime condizioni in cui versò. Ma la vocazione allo studio era stata più forte dei tristi eventi in mezzo ai quali si era venuto a trovare, e la fede nella fecondità delle idee sinceramente dibattute non era stata distrutta dall'avvilimento della temporanea sconfitta.

Le opere di questo periodo si possono dividere in due gruppi: quelle della prima fase sino al 1931-32, comprendenti studi di critica del fascismo nei suoi vari aspetti; quelle della seconda che mirano all'elaborazione di una completa dottrina del diritto e dello stato. Queste due fasi corrispondono non soltanto ad un naturale sviluppo dell'itinerario mentale del Trentin, ma anche all'evoluzione della situazione politica che corre dall'era cosiddetta di Locarno, l'era di liquidazione della guerra e di arginatura di nuove avventure, a quella della preparazione sempre più precipitosa della seconda guerra mondiale, che induce a non più attardarsi a criticare le istituzioni presenti in nome di quelle passate, ma formulare idee per l'assetto futuro.

La prima opera dell'esilio uscì, dopo due soli anni di soggiorno in Francia, nel 1928, e reca il titolo: L'aventure italienne. Légendes et réalités ${ }^{9}$. Non si tratta di un comune pamphlet antifascista: il libro vuol essere la dimostrazione documentata di una tesi. La tesi è questa: il fascismo ha ingannato e continua a ingannare gli italiani, ed anche gli stranieri, facendo credere due cose: la prima che abbia salvato l'Italia dal pericolo bolscevico, la seconda che l'abbia salvata dalla rovina economica. Queste affermazioni sono leggende. Trentin sostiene contro la prima che il periodo acuto di crisi delle istituzioni democratiche aveva avuto luogo nel 1920, e all'avvento del fascismo era ormai superato; contro la secon$\mathrm{da}$, mostra con documenti l'imponente opera di risanamento economico operata dai governi democratici negli anni del dopoguerra attraverso una politica economica avveduta e coraggiosa. Non il ristabilimento delle istituzioni ha recato il fascismo, ma la distruzione sistematica dello stato liberale; non il risanamento economico, ma la più completa rovina delle finanze statali. Non ancora del tutto chiarito era il problema delle origini del movimento fascista. Se la democrazia era sana in Italia, se una vena profonda di democrazia vi era nella vita pubblica italiana - e qui la polemica è diretta contro coloro che sbrigativamente attribuivano

${ }_{9}$ Con prefazione di M. A. Aulard, Presses Universitaires de France, Paris 1928, pp. 332. 
l'imporsi del fascismo alla mancanza di un'educazione democratica degli italiani -, come mai questo movimento antidemocratico, consacrato alla violenza, che professa disdegno per la teoria e antepone al pensiero l'azione, acquista così rapidamente terreno e prende alla fine il sopravvento? Questa prima interpretazione del Trentin tendeva a vedere nel fascismo un fenomeno contingente, dovuto alla crisi del dopoguerra che sorprese un organismo in crescenza, e quindi a considerarlo - come già indica il titolo - un'avventura, addirittura una effimera avventura, a cui aveva dato mano, e qui l'annotazione diventava più precisa, la borghesia, soprattutto l'alta borghesia impaurita dall'avanzarsi del proletariato.

Quest'ultima tesi veniva ripresa e approfondita in un secondo libro, intitolato Aux sources du fascisme ${ }^{10}$. L'opinione che il fascismo fosse la conseguenza di una stentata vita democratica in Italia, a cominciare dal $\mathrm{Ri}$ sorgimento, moto di élites che fanno violenza all'arretratezza della vita pubblica italiana, era penetrata anche nel gruppo degli emigrati. Il Trentin vi reagisce con veemenza. Sin dalle prime pagine il suo saggio appare diretto contro la tesi che il fascismo sia «un prodotto caratteristico del genio del popolo italiano, come la consacrazione logica, inevitabile, fatale, delle sue tendenze, della sua educazione, delle sue tradizioni, della sua storia $»^{11}$. Anzitutto, se pur il Risorgimento fu moto di élites, ai gruppi dirigenti del moto parteciparono tutte le classi sociali. Quanto all'Italia dopo l'unificazione - l'influsso della Storia d'Italia del Croce (che era apparsa nel 1928) è visibile - la classe dirigente non fu meno democratica di quella delle altre nazioni, e fu, se mai, meno meschinamente nazionalistica, e l'opinione pubblica fu sempre pronta a reagire agli abusi. E ancora una volta il problema: "perché dunque il fascismo?" veniva risolto con annotazioni sociologiche e psicologiche, come l'eccesso di fatica di un organismo indebolito dallo sforzo bellico, come un movimento di debolezza o di scoraggiamento. Ma per quanto non vi si insistesse, anche qui non mancava l'accenno alla contraddizione in cui si era trovata la borghesia tra i suoi ideali democratici e la paura delle nuove classi in ascesa, e il riconoscimento che la durata del fascismo (dopo quasi dieci anni non si poteva evidentemente più parlare di un fenomeno effimero) dipendeva prevalentemente dall'appoggio dei gruppi finanziari internazionali e della Chiesa.

Alla politica estera del fascismo è dedicato il libro uscito l'anno seguente: L'Italie à Genève ${ }^{12}$. Vi si dimostra che lo sforzo unico del fascismo è di mettere scompiglio nelle relazioni internazionali, di far fallire ogni piano di assestamento, di minare ogni tentativo volto all'attenuazione

\footnotetext{
${ }^{10}$ Marcel Rivière, Paris 1931, pp. 212.

${ }^{11}$ Ivi, p. 10.

12 Marcel Rivière, Paris 1932, pp. 26.
} 
della sovranità nazionale: una politica di guerra che ottiene un immeritato premio dalla colpevole debolezza della Società delle Nazioni. Allo stesso gruppo appartiene un'opera di qualche anno più tardi: Dalla bonifica integrale al pane dell'Impero. Dieci anni di fascismo totalitario. Stampata a Parigi, fu distrutta dai tedeschi ed ora è irreperibile. Vi appartengono pure alcuni scritti minori su aspetti particolari della politica fascista, come il codice penale e l'amnistia del ' $32^{13}$.

Lopera più importante di questa prima fase - forse una delle opere più durature del Trentin - esce ormai dai limiti della polemica politica e affronta con rigore giuridico il problema di fondo dello stato fascista. Reca il titolo: Les transformations récentes du droit public italien. De la Charte de Charles-Albert à la création de l'État fasciste ${ }^{14}$. È un grosso volume di circa 700 pagine, a cui premise una bella prefazione un autorevole giurista come il Bonnecase. L'opera, che meriterebbe di essere assai più nota, è un trattato di diritto costituzionale italiano, in cui si espongono successivamente, con l'intenzione di metterne in rilievo le differenze, il sistema costituzionale vigente in Italia prima del fascismo e quello introdotto nel fascismo con le varie leggi emanate dal 1925 al 1928. Molti costituzionalisti italiani, anche tra $i$ più eminenti, nascondendosi dietro il paravento del tecnicismo, si erano dati ad accogliere con la impassibilità del tecnico che non si mescola con la politica, le novità costituzionali fasciste e a inserirle nel sistema dello stato di diritto come se si fosse trattato di uno sviluppo organico del vecchio statuto bisognoso di rinnovamento. La sostanza eversiva delle nuove leggi veniva mascherata da benevole formule giuridiche. E se queste, per un verso, servivano a ritardare e ad affievolire l'asprezza del mutamento, a differenza di quel che accadde in Germania all'avvento del regime nazista, allorché si vide la scienza giuridica abbandonare frettolosamente gli schemi tradizionali e fare di ogni aberrazione politica una teoria filosofica e giuridica, per altro verso contribuirono a far apparire gradevole o per lo meno non terribile il truce volto del dispotismo. Il libro del Trentin è un atto di protesta e di accusa contro i giuristi asserviti, e insieme una documentata dimostrazione del loro errore: attraverso l'esame minuzioso dei singoli organi costituzionali, dopo le manipolazioni fasciste, egli mostra che il nuovo stato è l'antitesi dello stato di diritto, e deriva la sua sostanza ideologica dalle dottrine reazionarie affermanti la supremazia dello stato sull'individuo e la riduzione dell'individuo a strumento dei fini dello stato.

Ma ormai col passar degli anni il fascismo appariva fenomeno assai più grave di quel che fosse apparso ai primi oppositori. Anzitutto non

${ }^{13}$ Le Code pénal fasciste, LIDU, Paris 1932 e La mystification de l'amnistie fasciste, ESIL, Marseille 1933.

${ }^{14}$ Marcel Giard, Paris 1929, pp. XXXIII-696. 
era più un fenomeno soltanto italiano: sintomi di reazione fascista contro le istituzioni democratiche erano ben visibili anche altrove. E ciò dava ragione al Trentin che sin dall'inizio aveva confutato coloro che volevano spiegare il fascismo con motivi peculiari alla storia d'Italia. In secondo luogo si vedeva sempre più chiaramente quello che anche il Trentin aveva intuito, ossia che il fascismo era ovunque legato agli interessi di classe della borghesia, che barattava l'ideale di libertà con la tutela dei propri privilegi. Dopo il 1930 la politica europea ebbe un nuovo corso: la grande crisi economica, il graduale estinguersi delle speranze in una sistemazione pacifica dei rapporti tra Francia e Germania, (la fortunata ascesa di Laval in Francia nel gennaio 1931, la strepitosa vittoria nazionalsocialista nelle elezioni del '32, i tre giorni di sangue a Vienna nel febbraio del '34, che prepararono la dittatura di Dollfuss). La situazione interna e quella internazionale si andava inasprendo. Il fascismo avanzava. Qual destino era riservato ai regimi democratici?

Non è da stupire che un uomo come il Trentin, che aveva così intensamente sofferto la crisi italiana, fosse portato a porsi il problema della crisi che era non più soltanto italiana ma europea. Nel problema più vasto della crisi europea, il problema del fascismo diventava secondario, e si presentava al suo posto un'altra domanda: che cosa sarà dell'Europa dopo il fascismo? A ben guardare, le nuove meditazioni sulla crisi che egli andò facendo su per giù tra il '31 e il '33 - gli anni che segnano, come ci appare sempre più chiaro oggi a distanza di tempo, una svolta decisiva nella politica europea - accompagnano un suo profondo travaglio personale. Egli, per così dire, entrò nel vivo della crisi europea partecipandovi con una sensibilità acuita da una crisi interiore. E ne uscì con un sistema di principi che pur innestandosi sulle sue idee precedenti era profondamente rinnovato. Anzitutto, quanto al metodo, egli abbandonava ogni cautela legalitaria, e accettava il principio del rinnovamento mediante la rivoluzione. In secondo luogo, quanto alla sostanza, rigettava il radicalismo democratico, a cui si era fino allora ispirato, e abbracciava risolutamente il socialismo. È difficile dire quali ragioni lo abbiano condotto a questo nuovo atteggiamento. A giudicar dagli scritti, direi che due fenomeni sopra ogni altro lo colpirono: da un lato la debolezza delle democrazie parlamentari, reggentisi su un'economia a base individualistica, nell'opporsi all'aggressiva politica del fascismo, debolezza di cui soffrivano anche i vari partiti socialisti sia di destra sia di sinistra; e dall'altro la stabilità apparentemente solida del regime sovietico alla fine del primo piano quinquennale. Certo è che nella sua prima opera di questa seconda fase, scritta in italiano e intitolata Riflessioni sulla crisi e sulla rivoluzione ${ }^{15}$, la sfiducia nei regimi democratici borghesi e l'ammirazione, se pur circondata

15 Marseille, ESIL, senza data, ma 1933, pp. 155. 
da sostanziali riserve, per l'Unione Sovietica, sono ormai energicamente dichiarate. Ancor nell'opera di due anni prima, Aux sources du fascisme, formulando a guisa di conclusione qualche proposta per la salvezza della democrazia, condannava i monopoli che voleva sostituiti da uffici pubblici autonomi e difendeva le libertà locali contro lo stato accentrato, dando prova di non aver oltrepassato gli ideali della democrazia radicale. Nella nuova opera, invece, nettissima è l'affermazione che l'ordine nuovo non potrà essere realizzato se non rivoluzionariamente (cioè con espropriazioni senza indennizzo delle fabbriche, delle terre, delle banche, ecc.); e altrettanto netta l'altra affermazione che questo ordine deriverà dalla trasformazione del sistema di appropriazione capitalistica in quello collettivistico. L'esempio di ciò che ha fatto la Russia, dopo la rivoluzione, costituisce il nerbo del libro, massime l'insegnamento, che se ne deve trarre, intorno all'attitudine dello stato a dirigere l'economia. Si arrischia addirittura la profezia che la rivoluzione italiana dovrà chiedere soccorso, per raggiungere la propria meta, alla rivoluzione russa. La grossa riserva che egli muove al regime sovietico riguarda il rispetto della persona: dichiara di essere un rivoluzionario liberale, e l'abisso, egli dice, che separa la Russia dal regime a cui tendono i rivoluzionari liberali, si chiama libertà. La sua professione di fede è contenuta in queste parole, poste al principio del libro: «È vano pretendere di poter transigere impunemente con il metodo della libertà, perché le esigenze di questo sono e restano categoriche e irriducibili. Non vi è certezza o verità umana che possa rivendicar la prerogativa di ignorarle o di disconoscerle» ${ }^{16}$. Ma come si realizza la libertà in un regime di collettivismo economico? La risposta è un programma: attraverso l'autonomia, intesa come l'organizzazione autosufficiente dei singoli gruppi, territoriali e non, che compongono lo stato. È evidente che su questo punto Trentin risuscitava la sua antica fiducia di studioso di diritto amministrativo per l'autogoverno locale, e correggeva con la sua esperienza di giurista, sempre diffidente di fronte allo stato accentratore, le conseguenze che era andato traendo dalle considerazioni sulla crisi economica.

Il suo pensiero si muoveva ormai in due direzioni: sul terreno economico, verso il collettivismo; sul terreno politico-giuridico, verso lo stato pluralistico. L'un concetto rappresentava l'antidoto dell'altro. Quel che vi era di minaccioso per la libertà individuale nel collettivismo doveva essere attenuato dal sistema delle autonomie; quel che vi era di iniquo nel sistema dell'economia liberale doveva essere superato dal sistema collettivistico. Di fronte alla crisi che spaccava il mondo in due parti, e poneva i problemi in termini di dure alternative, o di qua o di là, il Trentin, con un atteggiamento che è caratteristico dell'intellettuale in tempo di

16 Ivi, p. 17. 
ribollimento sociale, cercava una sintesi. Chiamiamo i due termini dell'alternativa coi nomi noti di giustizia e libertà, ed erano del resto i nomi del movimento politico cui egli appartenne sin dalla costituzione (1929): Trentin non esitava ad accettare la prima nella sua formulazione più rigorosa, ma non rinunciava ad escogitare espedienti per la seconda, che si riassumevano nel termine, affascinante, di autonomia. Di due modi diversi di cercare la sintesi, che affiorava nel movimento di Giustizia e Libertà, e si scontravano anche in sede pratica, l'uno più prammatico, rappresentato da Carlo Rosselli, consisteva nella attenuazione e reciproca integrazione dei due astratti principi, che venivano così a perdere della loro rigidità; l'altro più dottrinario, e quindi più rigido, consisteva nel mantenere la contrapposizione dei due principi e nel trovare un sistema in cui avessero attuazione contemporaneamente tutti e due nella loro purezza, Trentin rappresentò, meglio forse di ogni altro, questo secondo modo. In un articolo pubblicato sui "Quaderni di Giustizia e Libertà» nel 1934 $4^{17}$, intitolato Bisogna decidersi, poneva in questi termini la questione: o regime intermediario fra capitalismo e collettivismo, implicante un'economia a due settori (che era la tesi quasi ufficiale di Giustizia e Libertà) o regime collettivistico. Affermava che il movimento doveva decidersi per il secondo termine, e che pertanto doveva perseguire insieme coi comunisti gli stessi fini nel campo delle realizzazioni concrete pur distinguendosi da essi nella aspirazione alla libertà. Per questa sua intransigenza dottrinale appartenne all'ala sinistra del movimento e la sua azione fu caratterizzata da una simpatia per la politica del Fronte Popolare, che non era condivisa dalla maggior parte dei suoi amici ${ }^{18}$.

Le idee avanzate nel libro Riflessioni sulla crisi e sulla rivoluzione trovano compiuta elaborazione teorica nella voluminosa opera che uscì nel 1935 col titolo: La Crise du Droit et de l'État, con prefazione di uno dei più illustri giuristi francesi, François Gény ${ }^{19}$. Qui appare per la prima volta il concetto, se non ancora una teoria compiuta, del federalismo. Il principio dell'autonomia, già affermato nell'opera precedente, e chiamato a far da contrappeso al principio dell'uguaglianza economica, si viene chiarendo come il concetto chiave di una teoria federalistica della società: decisivo fu l'influsso del pensiero di Proudhon, e non estranee le suggestioni della teoria del diritto sociale del Gurvitch, cui vengono dedicate in questa nuova opera molte pagine, se pur di dichiarato dissenso. L'impianto dell'opera è il più ampio possibile: si risale ai fondamenti stessi del diritto, si discorre della nozione del diritto, dei rapporti fra diritto e

17 Fasc. XI, pp. 99-109.

18 Si veda A. Garosci, Storia dei fuorusciti, Laterza, Bari 1953, pp. 169, 183, 206, 220, 247.

19 Alcan, Paris 1935, pp. 493. 
stato, si affronta il problema del diritto naturale. È un saggio di filosofia del diritto e dello stato nel senso più proprio dell'espressione, sia per i problemi trattati, sia per gli autori citati, sia per le soluzioni abbozzate. Il disegno è grandioso: vi si delinea la lotta fra l'individuo che è il centro vitale e morale della società e come tale non può essere mai totalmente assorbito nel gruppo, e lo stato moderno che, pur partendo da premesse individualistiche, sta esaurendosi ingloriosamente, vittima dell'economia capitalistica, nelle attuali dittature. Grandeggia visibilmente in tutto il libro l'ideale democratico non mai abbandonato, inteso come ideale dell'emancipazione della persona umana dai vincoli che di volta in volta la opprimono nella storia. La realizzazione di questo ideale ha bisogno al tempo presente di due condizioni fondamentali: 1) la liberazione economica dell'individuo attraverso la soppressione della proprietà privata; 2) la liberazione politica attraverso il federalismo.

D'ora innanzi l'accento della teoria e dell'azione di Trentin vien posto sul motivo politico federalistico ancor più che su quello economico socialista. Sulla soluzione del problema economico non ha più dubbi: la corruzione del mondo borghese, l'appoggio che esso ha cercato nella dittatura, lo hanno illuminato con una certezza che non soffre smentite. Resta il problema vivissimo, ineludibile per una coscienza liberale come la sua, d'impedire che la soluzione che gli sembra ormai così ovvia, avvenga per vie, con mezzi e con risultati contrari allo spirito della libertà. La struttura federalistica della società, che spezza il monopolio dello stato monocentrico, gli si presenta come il rimedio decisivo. Vi dedica nel 1940 un'opera che uscirà postuma, in edizione clandestina, per i tipi della casa editrice "La Fiaccola" di Milano, nel 1945, con prefazione di Mario Dal Pra: Stato, nazione, federalismo; quindi un più breve saggio, rimasto inedito, intitolato Libérer et Fédérer. La prima contiene soprattutto una critica storica dello stato moderno, sia esso monarchico o democratico, in quanto stato accentrato, e questa critica si prolunga sino agli scopi di guerra delle potenze belligeranti, le quali hanno dimostrato di non aver imparato la lezione della storia. E conclude con l'affermazione che solo una rivoluzione bivalente, cioè insieme anticapitalistica e federalistica, potrà salvare insieme con l'Europa la libertà. Il secondo spiega concisamente la formula del titolo, che era divenuto nel frattempo il nome di un movimento clandestino da lui fondato, che partecipò alla resistenza francese: "Liberare", cioè emancipare economicamente l'individuo attraverso la distruzione dello stato capitalistico e di tutte le sue successive ingannevoli incarnazioni; "federare", cioè emancipare politicamente l'individuo attraverso la distruzione dello stato totalitario.

Che cosa sia stato di questi ideali non è qui il caso di dire né di lamentare. E se qualcosa di meccanico appare nella contrapposizione dei due termini di giustizia e di libertà, e di astratto nella soluzione, bisogna riportarsi a quei tempi, a quella ricerca di nuovi modelli di vita sociale che 
era accompagnata, del resto, da un ardente fervore nell'azione realizzatrice, e se ne vedrà il profondo significato normativo che, a mio avviso, non è venuto meno neppure oggi. Sul piano teorico c'era in quella formula il proposito di accogliere un fatto gigantesco come la rivoluzione sovietica, di fronte al quale era cattiva politica chiudere gli occhi, pur inserendolo negli schemi del pensiero politico occidentale; sul piano pratico, il consiglio a non dimenticare che emancipazione politica ed emancipazione economica devono procedere di pari passo, e che là dove la rivoluzione economica spinge verso il livellamento la rivoluzione politica deve, per contrappeso, spingere verso la differenziazione. Del resto, tutte le teorie politiche, e non solo quelle utopistiche, non coincidono mai, proprio in quanto teorie, con la realtà. La loro fecondità non si misura col metro della maggiore o minore approssimazione, o del maggiore o minore spazio di tempo, in cui diventano realtà; ma tenendo presente lo stimolo che esse danno all'azione in una certa situazione storica, ovvero la loro capacità di trasformarsi o meno in regole di azione. L'ideale della lotta su due fronti, contro lo stato borghese in nome dell'eguaglianza, contro lo stato totalitario in nome della libertà, fu uno dei più validi motivi di lotta contro il regime fascista che era insieme borghese e totalitario. Fu il motivo di lotta - di cui la dottrina di Trentin si può considerare una formulazione, se pur non la sola, - che caratterizzò, com'è noto, il Partito d'azione, cui egli, nel breve soggiorno in Italia, aderì.

Il ritorno in Italia avvenne, dopo quasi diciotto anni di esilio, ai primi di settembre del 1943, quando stava per spirare il breve periodo di libertà provvisoria, che giudici frettolosi e malaccorti avevano concesso al popolo italiano. Dopo pochi giorni cominciava la guerra civile, e continuava per lui, ancor più aspro e tormentato, l'esilio. Allora lo incontrammo, e l'incontro diede più forza, più speranza, quasi un suggello di nuova dignità, alla nostra azione. Si erano costituiti i primi nuclei di resistenza e l'Università di Padova era uno dei centri più attivi. Tornandovi, egli ritornava nella sua casa, non come il maestro riconosciuto, ma come un ignoto personaggio che vi capiti e vi si aggiri per caso. E confuso tra la folla assistette, condottovi da un amico, all'apertura dell'anno accademico, quando Concetto Marchesi sfidò gli effimeri e torvi padroni inaugurando l'anno degli studi in nome dell'Italia dei lavoratori. Negli incontri, in cui si ponevano le basi della organizzazione clandestina, recava il contributo della sua esperienza, delle sue idee a lungo maturate e profondamente radicate nel suo spirito, e della sua ferma coscienza. Lo riconoscemmo immediatamente come la guida che avevamo cercata. Ricordo la serenità del suo volto che incuteva rispetto, quella austerità del comportamento che dava e chiedeva fiducia, il passo sicuro che era il segno di una conquistata calma interiore e di consapevole coraggio. La sua presenza ci offriva un incitamento per il presente e una certezza per l'avvenire. Quale avvenire? Quello ch'egli si era raffigurato nelle 
sue meditazioni sulla crisi e sulla rivoluzione? Al di là dei progetti per il futuro assetto dello stato, c'era in quei giorni una meta ben chiara e più immediata: la liberazione. Ed egli vi si dedicò con impeto e con l'imperioso dominio, che gli era naturale, di sé stesso e degli eventi.

E la meta è stata raggiunta. Ma non da lui che l'aveva attesa per lunghi anni. Il destino gli serbò del ritorno in patria la sofferenza della schiavitù ribadita e inasprita, non la gioia della rinascita. Non appena i nuovi fascisti si furono organizzati, fu arrestato e tenuto in prigionia per qualche settimana. Quando ne uscì, il suo organismo era devastato. Fu costretto a ritirarsi in una clinica dove, dopo alcuni mesi di infermità e di mal sopportata inerzia, morì il 12 marzo 1944. Gli fu data sepoltura di sera, nascostamente, vigilata la bara da guardie sospettose ed inquiete. Moriva nella notte, per lui senza fine, dell'esilio, mentre prossima era l'alba del nuovo giorno. Moriva nella solitudine, lontani e dispersi gli amici, quelli con cui aveva diviso gli anni del distacco, quelli che si era conquistati negli ultimi mesi. Ma uno di essi - il più degno - Egidio Meneghetti, lo ricordò in un accorato necrologio che era insieme una veemente invettiva, apparso, chi lo rammenta ancora?, in caratteri minutissimi nell'ultima pagina del giornale clandestino "Fratelli d'Italia», che recava la data del 15 maggio 1944.

Lontani e dispersi, allora, gli amici, ma ora, dopo dieci anni, accanto all'eletta consorte e ai figli dilettissimi, ricongiunti, i morti e i superstiti, insieme con tutti i devoti della libertà, per ricordarlo, e ricordandolo dire: «Conserviamo nella mente l'immagine del suo volto sereno e forte. Cerchiamo di restar fedeli alla nobiltà del suo esempio. Tramandiamo ai giovani la memoria del suo animo incorrotto. Ne abbiamo ancora bisogno». 


\section{SILVIO TRENTIN E LO STATO FASCISTA*}

Come primo volume delle Opere scelte di Silvio Trentin è apparsa, nel 1983, presso l'editore Marsilio di Venezia, a cura e con un'ampia, dotta e impegnativa introduzione di Alessandro Pizzorusso, la traduzione italiana dell'opera Les transformations récentes $d u$ droit public italien (pubblicata a $\mathrm{Pa}$ rigi nel 1929), col titolo Dallo Statuto albertino al regime fascista. Trentin era in esilio in Francia dal febbraio 1926. Vi era giunto con la famiglia dopo aver dato in gennaio le dimissioni da professore ordinario di Istituzioni di diritto pubblico nell'Istituto superiore di scienze economiche e commerciali di Venezia, in seguito all'emanazione del decreto-legge 24 dicembre 1925 che attribuiva al governo la facoltà di dispensare dal servizio i funzionari che non dessero "piena garanzia di un fedele adempimento dei loro doveri» e si ponessero «in condizioni d'incompatibilità con le generali direttive politiche del governo».

L'opera ora tradotta in italiano appartiene al primo gruppo di scritti francesi che Trentin dedica all'analisi giuridica, storica, politica del fascismo. È preceduta da L'aventure italienne (1928) e seguita da Antidémocratie (1930), Aux sources du fascisme (1931), Le fascisme à Genève (1932). Fu scritta tra la primavera del 1928 e il gennaio 1929. Avrebbe dovuto presentarla al pubblico francese con una prefazione Maurice Hauriou, doyen della facoltà giuridica dell'Università di Tolosa, uno dei più autorevoli, e meritatamente celebri, giuristi francesi del tempo, se non fosse morto dopo breve malattia nel marzo dello stesso anno. Scrisse la prefazione Julien Bonnecase, che era professore di diritto all'Università di Bordeaux, autore di due opere molto note (se pure anche molto discusse), L'école de l'exégèse en droit civil ( $2^{\mathrm{a}}$ ediz. 1928), e Science du droit et romantisme (1928).

* Silvio Trentin e lo Stato fascista, «Belfagor», XL (6), 30 novembre 1985, pp. 700-707.

${ }^{1} \mathrm{~S}$. Trentin, Dallo statuto albertino al regime fascista, a cura e con introduzione di A. Pizzorusso, Marsilio, Venezia 1983. Il progetto della pubblicazione delle opere di Silvio Trentin è stato proposto dal Centro di ricerca Silvio Trentin di Jesolo; l'iniziativa è stata presa dalle università di Padova, Pisa, Venezia, dal Centro studi Piero Gobetti di Torino e dall'Istituto veneto per la storia della Resistenza di Padova. [Cfr. la Bibliografia di e su Silvio Trentin in appendice]. 
L'importanza di questo libro consiste nell'essere l'unica trattazione scientifica, come scrive Pizzorusso, «dedicata al sistema costituzionale creato dal fascismo da uno studioso non sottoposto alle limitazioni imposte dal regime alla libertà di pensiero $»^{2}$. Ma proprio per questo non fu degnato allora da parte dei giuristi italiani né di una citazione né di una critica. L'autore ne mandò qualche copia alle biblioteche italiane (ne esiste una nella biblioteca dell'Istituto giuridico della facoltà di giurisprudenza di Torino), ma quasi tutte le biblioteche universitarie, così scrive Pizzorusso, ne sono prive. La presente traduzione rende possibile a più di cinquant'anni di distanza la riscoperta di un documento prezioso per la storia del diritto pubblico in Italia, e ancora più in generale per la storia delle interpretazioni del fascismo. Si tratta in parte di una storia costituzionale italiana dopo l'Unità, in parte di una critica delle leggi costituzionali e dei loro pedissequi commentatori fascisti, in parte anche di un tentativo di rintracciare dietro le innovazioni legislative l'ideologia che le aveva ispirate. Il principale bersaglio dal punto di vista ideologico è Alfredo Rocco, la cui raccolta di discorsi politici, pubblicata nel 1927, col titolo La trasformazione dello stato, può aver suggerito a Trentin il titolo della propria opera, anche se non è da escludere la reminiscenza del notissimo libro di Léon Duguit, un giurista che egli stima pur criticandone spesso l'eccessivo radicalismo filosofico, Les transformations $d u$ droit public français. Trentin fu uno dei pochi, o forse il solo, degli italiani in esilio dotato di una profonda cultura giuridica. L'opera principale che egli scrisse in Francia, ancora meno conosciuta e ancora più rara nelle biblioteche italiane, è una trattazione di filosofia del diritto, La Crise $d u$ Droit et de l'État, pubblicata nel 1935, con una prefazione di un altro illustre giurista francese del tempo, François Gény.

Quando l'opera sul diritto pubblico fascista apparve in Francia, io ero studente del secondo anno di giurisprudenza all'università di Torino. Fu l'anno in cui diedi l'esame di diritto costituzionale, la materia stessa del libro. Ma il diritto costituzionale che noi studiavamo era quello esposto commentato giustificato dai molti ortodossi, non quello criticato dall'unico eretico. Che il diritto costituzionale italiano fosse stato trasformato né gli ortodossi né l'eretico potevano mettere in dubbio. Tra gli uni e l'altro ciò che cambiava era il giudizio di valore: per i primi era stato trasformato in meglio, per il secondo in peggio. Per Rocco il fascismo aveva finalmente instaurato un ordinamento «integralmente e nettamente italiano» sostituendolo a quello liberale che l'Italia aveva importato servilmente da altri paesi; per Trentin, invece, era riuscito in pochi anni a stravolgere l'ordinamento liberale che aveva accompagnato la nascita e il primo sviluppo dello stato unitario. Per gli ortodossi il fascismo non

2 Ivi, p. IX. 
solo non aveva stravolto lo Statuto albertino (che si era trasformato, sì, nei cento anni della sua storia, ma entro la cornice dello stato liberale e di diritto), ma lo aveva integrato, arricchito, rigenerato, rinnovato, ammodernato e avviato a nuovo e fulgido avvenire. Per l'eretico, non solo lo aveva stravolto, ma lo aveva capovolto, perché aveva a poco a poco trasformato-deformato un ordinamento liberale, se pure con tutti i suoi limiti, e democratico (nel 1919 era stato introdotto il suffragio universale maschile), in una vera e propria dittatura, in un governo, per usare la categoria di Max Weber, già formulata ma non ancora diffusa nella nostra cultura, del capo carismatico.

Il nostro professore di diritto costituzionale era un vecchio studioso di buona fama, ancora vivacissimo nonostante gli anni (era nato nel 1857), docente appassionato ed efficace, succeduto a Gaetano Mosca, chiamato all'università di Roma nel 1925 a insegnare la Storia delle dottrine politiche ${ }^{3}$. Come gran parte o forse tutti i costituzionalisti italiani era stato un leale fautore e difensore dello stato liberale e dello stato di diritto, sulla cui legittimità non erano mai sorti seri dubbi. Vi potevano essere giuristi più conservatori o più progressisti, ma i fondamenti dello stato liberale di diritto non venivano messi in discussione. Il nostro professore era autore di un manuale di Istituzioni di diritto costituzionale italiano, uscito nel 1913 presso l'editore torinese Bocca, che aveva avuto una notevole fortuna, più volte citato dallo stesso Trentin, e poco dopo aveva dato alle stampe una monumentale Storia costituzionale del Regno d'Italia, allora, in parte ancor oggi, unica nel suo genere (un genere in quegli anni poco coltivato nel nostro paese). Per gli studenti del primo anno insegnava una materia da tempo scomparsa nelle nostre facoltà (in parte reintrodotta nell'ordinamento delle facoltà di scienze politiche), che si chiamava Introduzione allo studio della scienza giuridica e istituzioni di diritto pubblico. Mi sono andato a rivedere le dispense del 1927, quelle sulle quali preparai l'esame: con mia sorpresa e, devo dirlo, anche con un sentimento di gratitudine per il vecchio maestro, del fascismo ne verbum quidem. Il metodo astratto, formalistico, proprio della scuola cosiddetta del tecnicismo giuridico, della nuova scuola venuta dalla Germania, che aveva separato il diritto pubblico dalla scienza politica, e aveva adottato il metodo cosiddetto dommatico che tanto successo aveva avuto nel diritto privato, permetteva di descrivere un sistema giuridico, in specie il sistema italiano del diritto pubblico, nelle sue partizioni fondamentali - legislazione, amministrazione, giurisdizione - senza riempirlo di contenuti storici. Sarebbe arrivato in quegli stessi anni il grande Kelsen (che Trentin mostra di conoscere molto bene, anche se non ne accetta il nudo e crudo 1936].

[Gaetano Arangio Ruiz, Augusta (Siracusa), 18 aprile 1857-Torino, 30 luglio 
formalismo) a sostenere che la dottrina pura del diritto costruisce l'ordinamento giuridico come sistema dinamico di norme tale da renderlo idoneo a descrivere tanto uno stato liberale quanto uno stato socialista.

Naturalmente questa indifferenza al contenuto era un atteggiamento che non poteva essere assunto nell'insegnamento del diritto pubblico italiano, il cui compito era di esporre e commentare la costituzione positiva e vigente. Ormai nel 1929 la costituzione positiva e vigente in Italia non è più lo Statuto albertino né nella sua forma originaria né nella forma che aveva ricevuto a poco a poco nel corso quasi secolare della sua lenta evoluzione. Con la legge del 9 dicembre 1928 sull'ordinamento e le attribuzioni del Gran Consiglio l'edificio costituzionale fascista, le cui basi erano state poste tre anni prima dalla legge 24 dicembre 1925 sulle attribuzioni e prerogative del capo del governo, poteva dirsi compiuto. Compiuto, anche se il processo di trasformazione-deformazione non si arresterà a questo punto, ma arriverà sino alla istituzione della Camera dei Fasci e delle Corporazioni (1939) che eliminerà anche quella parvenza di regime parlamentare rimasta con le elezioni plebiscitarie del 1929 e del 1935, e la riduzione del Senato a luogo di acclamazione. Il nostro professore non poteva più evidentemente farci studiare il diritto nuovo nel suo vecchio libro del 1913. Dovette, con suo dispetto, mettere frettolosamente insieme delle dispense che furono pubblicate quello stesso anno e avevano il vantaggio per noi studenti di essere molto più brevi (le Istituzioni citate erano un volumone di 600 pagine). Ciò che caratterizzava il nuovo corso era, per usare un'espressione del linguaggio giuridico tradizionale, la recezione delle innovazioni, e la loro neutralizzazione.

Con l'andar del tempo, e col progressivo consolidamento del regime, l'atteggiamento prevalente nella nostra dottrina pubblicistica fu generalmente quello di considerare la riforma fascista dello stato come una serie (coerente e organica) di innovazioni, sì, ma nella continuità. Quale continuità? $\mathrm{Ma}$ - era chiaro - la continuità dello Statuto albertino (se non proprio dello stato liberale su cui si facevano ricadere tutte le colpe della crisi della società italiana nel dopoguerra). Diventò un fatto assolutamente normale che l'autore di un corso di diritto costituzionale prefascista pubblicasse un corso di diritto costituzionale fascista come una nuova edizione dello stesso libro, semplicemente riveduta e aggiornata. Del resto, lo stesso accadde nel passaggio dal regime fascista alla repubblica democratica. Il mio collega di diritto costituzionale a Torino, egregio studioso e vecchio gentiluomo, e anche mio buon amico ${ }^{4}$, un'amicizia che non ho alcuna ragione di rinnegare (ma chi fu veramente senza peccato?), aveva scritto uno dei più noti trattati di diritto costituzionale fascista, pubblicato dall'Utet nel 1947. Nel 1950 pubblicò la seconda edizione «riveduta».

${ }^{4}$ [Emilio Crosa, Torino, 1885-ivi, 1962]. 
Ma già nel 1946, quindi prima dell'elaborazione della Costituzione, anzi prima ancora che l'Assemblea costituente iniziasse i propri lavori, diede alle stampe un libro intitolato Lo stato democratico. Presupposti costituzionali, che reca la data del 22 settembre 1945. Nel quale il grande assente (assente perché rimosso) è il fascismo. Solo nelle ultime pagine viene sfatato il pregiudizio che al governo si debbano attribuire ampi poteri, specie al di fuori delle leggi, e si condanna «il malcostume politico, invalso con uno spaventoso crescendo nel periodo fascista, quando le stesse leggi volute dal regime all'infuori di ogni partecipazione popolare, erano dallo stesso governo e dai suoi organi sistematicamente violate" ${ }^{5}$.

Tra le varie interpretazioni del fascismo, due, com'è ben noto, sono prevalenti, quella della continuità, s'intende della continuità storica, che viene attribuita a Gobetti per la sua famosa definizione «il fascismo è l'autobiografia della nazione», e quella della parentesi, attribuita a Croce, che aveva paragonato il regime alla calata degli Hyksos. Ebbene per molti giuristi italiani (ma, siamo giusti, non solo per i giuristi, per molti intellettuali, e anche per gran parte degli italiani, negli anni cosiddetti del consenso) il fascismo fu l'una e l'altra cosa insieme: continuità, durante; parentesi... dopo. Continuità durante perché così esigeva la conservazione del proprio stipendio; parentesi dopo... per la stessa ragione.

L'importanza del libro di Trentin sta nel fatto, come si è detto all'inizio, che è l'unico trattato di diritto costituzionale fascista che dicesse pane al pane: no, il regime non era stato un'integrazione (benefica) ma una disintegrazione (malefica) dello stato liberale italiano. Ciò che se mai suscita stupore e sdegno nell'animo di Trentin è che la trasformazione nel senso forte (non blando) e regressivo (non progressivo) del termine fosse avvenuta "quasi naturalmente, senza alcuna reazione e senza alcuna resistenza ${ }^{6}$. Il libro di Benda, La trahison des clercs, era uscito nel 1927. Quale miglior conferma dei sopravvenuti anni del tradimento che il sacrificio delle proprie convinzioni, senza alcuna resistenza, offerto dai sacerdoti del diritto ai nuovi idoli? Ecco che cosa scrive Trentin in una delle pagine più aspre del libro, che non possiamo rileggere ora senza un senso di amarezza o di mortificazione:

Bisogna confessare - ed è questa una delle circostanze che più colpiscono soprattutto gli stranieri - che la scuola italiana non ha nemmeno cercato di difendere le sue prerogative, che essa si è docilmente allineata alla volontà del partito al potere, che essa ha consentito - senza nemmeno accennare a un gesto di rivolta - a trasformare tutto d'un colpo la libera cattedra in tribuna mercenaria destinata alla diffusione della verità ufficiale.

\footnotetext{
${ }^{5}$ Ivi, pp. 279-280.

${ }^{6}$ Ivi, p. 326.
} 
Questa "vergognosa abdicazione sembra tanto più inesplicabile, in quanto l'università italiana aveva una sua tradizione da difendere, e un nobile compito, ad essa esclusivamente spettante, da preservare» ${ }^{7}$. Dopo aver ricordato alcune nobili eccezioni, Salvemini, Arturo Labriola, Enrico Presutti (il primo aveva rinunziato alla cattedra come Nitti e lo stesso Trentin, il secondo e il terzo accettarono che fosse revocato il loro incarico d'insegnamento pur di non rinunciare alla loro libertà), continua: «La gran massa - la massa degli illustri e degli sconosciuti, degli anziani e dei giovani - consentirono subito con la migliore buona volontà a obbedire come un docile gregge [...] ai nuovi maestri d'Italia» ${ }^{8}$.

Di fronte a questo contrasto tra i pochi ribelli e i molti conformisti risorge il problema così spesso discusso in questi ultimi anni se sia esistita in Italia una cultura fascista. La mia risposta è sempre stata negativa, ma non ignoro che la communis opinio degli studiosi ha sempre sostenuto e difeso la tesi contraria, anche se, e me ne rallegro, un giovane storico francese, che ha scritto due buoni libri sul fascismo, e l'ultimo proprio sul tema degl'intellettuali, condivide in sostanza la tesi da me sempre pervicacemente sostenuta ${ }^{9}$. Una delle ragioni per cui non ho mai creduto sia esistita una cultura fascista sta anche nel fatto che l'accettazione della cosiddetta dottrina fu il prodotto non tanto di una convinzione quanto di un adattamento alle dure leggi di uno stato di polizia, e la spalmatina che ne derivò fu così superficiale che bastò una rapida raschiatura per cancellare la leggera patina. Non ho mai creduto che i nostri maggiori giuristi credessero veramente che lo stato fascista fosse la continuazione o addirittura l'inveramento dello stato liberale e dello stato socialista come sosteneva la Scuola corporativa di Pisa. Salvo poche eccezioni, come il prof. Carlo Costamagna, che aveva addirittura scimmiottato le dottrine naziste, ma non era tenuto in gran conto dai giuristi di buona scuola (rivalutato in questi anni dalla nuova destra) $)^{10}$, o come quello strano personaggio, nato dal nulla e finito tragicamente nel nulla, che fu Antonio Canepa, autore di un Sistema di dottrina del fascismo in tre volumi, di cui non resta memoria alcuna ${ }^{11}$. I giuristi di buona scuola si era no adattati

7 Ivi, p. 326.

${ }^{8}$ Ivi, p. 331.

${ }_{9}$ Mi riferisco ai due libri di M. Ostenc, L'éducation en Italie pendant le fascisme, Publications de la Sorbonne, Paris 1980, e Intellectuels italiens et fascisme, Payot, Paris 1983.

${ }^{10}$ Mi riferisco al manuale Dottrina del fascismo, ripubblicato dalla casa editrice della Tavola Rotonda nel 1982, senza indicazione di luogo. La prima edizione era apparsa in Germania col titolo Fascismus (Entwicklung und Lehre), quindi il libro fu stampato nel 1940 in Italia.

${ }^{11}$ Dal libro di G. Montalbano, Questione morale e separatismo, banditismo, mafia, Palermo 1985 (senza nome di casa editrice), ho appreso che il Canepa, palermitano, incaricato di storia dei trattati nell'Istituto di scienze economiche dell'Università di Catania, aveva tentato un colpo di stato insieme col fratello e con un gruppo di fuoriusciti sanmarinesi nel 1933 allo scopo di instaurarvi un regime democratico modello. 
al nuovo corso, non diversamente dalla maggior parte degli italiani, per amore del quieto vivere, cercando di salvare la propria anima con qualche acrobazia intellettuale oppure ammantandosi (e coprendosi) con formule tecniche, e così contrapponendo agli arcana imperii gli arcana sapientiae (non meno misteriosi e inaccessibili ai profani).

Che fossero acrobazie intellettuali, basti questo esempio. Nel 1940 uscirono quattro volumi destinati a onorare Santi Romano, che aveva accettato dal fascismo l'alta carica di presidente del Consiglio di Stato, ma era pur sempre il principale fautore della teoria della pluralità degli ordinamenti giuridici, che era la dottrina, quanto più possibile lontana dalla concezione monistica dello stato esaltata dal regime. Nel primo volume c'è un articolo di uno dei più noti costituzionalisti italiani, il vecchio Luigi Rossi (nato nel 1867), che come giurista e uomo politico non era il primo venuto, professore a Roma dal 1925, deputato per vent'anni dal 1904 al 1924, più volte ministro, e anche nell'ultimo governo liberale prima della Marcia su Roma, nel governo dell'on. Facta. L'articolo è intitolato, La elasticità dello Statuto, dove per «elasticità» non s'intende, come potrebbe sembrare a prima vista, il noto attributo della flessibilità, in base al quale si distinguono da Bryce e Dicey in poi le costituzioni rigide da quelle flessibili, ma «la caratteristica conformazione di una certa costituzione la quale agevolmente s'adatti alle variabili necessità dei tempi e delle circostanze, perché le sue formule, sintetiche e generiche, lasciano largo margine al loro sviluppo e alla loro integrazione mediante leggi costituzionali particolari, consuetudini e interpretazioni varie». Ora, secondo Luigi Rossi, uno dei caratteri distintivi dello Statuto albertino era proprio questa elasticità «in virtù della quale leggi costituzionali speciali hanno potuto modellare diversamente vari istituti [...] senza urtare contro la legge fondamentale». Di qua la stupefacente conseguenza che la «costituzione» si era andata continuamente modificando (ma così si mettevano sullo stesso piano gli sviluppi interni verificatisi durante l'era liberale e i nuovi istituti estranei imposti dal regime), mentre lo «statuto» era rimasto lo stesso. La contrapposizione fra il nostro Statuto che era rimasto in piedi e la costituzione di Weimar che era crollata di fronte al nazismo lo induceva a questa conclusione: «Senza fare della retorica sentimentale e romantica, si può affermare che lo Statuto è uno di quegli atti che dipendono da misteriose fatalità della storia e che, alla loro volta, la formano" ${ }^{12}$. Dove, l'unico vero mistero è nel come abbia-

Arrestato e condannato a 10 anni di lavori forzati, poi assolto dalla magistratura italiana per infermità mentale, diventò nel 1942, passato all'antifascismo, membro attivo del movimento separatista siciliano, collo pseudonimo di Mario Turri. Fu ucciso in un conflitto a fuoco dai carabinieri nel giugno 1945.

${ }^{12}$ L. Rossi, L'elasticità dello Statuto, in Studi in onore di Santi Romano, Cedam, Padova 1940, vol. I, pp. 25-43. 
no potuto, l'uno, lo Statuto, restare fermo, l'altra, la Costituzione, cambiare, quando statuto e costituzione sono due parole che vengono usate abitualmente, e anche nel linguaggio tecnico, per indicare la stessissima cosa. Ancora una volta credo che l'unico modo per spiegare queste forzature sia quello di interpretarle come effetti del fortissimo desiderio di auto-assoluzione. Pareto li avrebbe chiamati, questi maldestri tentativi di giustificare razionalmente o con una parvenza di argomentazione razionale l'ingiustificabile, "derivazioni», ovvero razionalizzazioni postume di sentimenti tanto forti da non potere essere soffocati, da potere essere soltanto addomesticati, edulcorati, repressi, sublimati.

Bisogna anche dire che questo adattamento al regime da parte dei giuristi fu favorito dalla concezione della scienza giuridica, e quindi anche del compito del giurista nella società, propria del positivismo giuridico, secondo cui tutte le scienze, e pertanto anche la giurisprudenza, se ha da essere scienza, debbono essere avalutative, l'oggetto della scienza giuridica essendo il ius conditum e non il ius condendum, e il ius conditum è il diritto posto dal potere sovrano, giusto o ingiusto che sia. E che lo stato fascista, in quanto stato a pieno diritto, fosse sovrano, e il diritto fascista fosse a pieno titolo diritto positivo, in base al principio di effettività, non poteva essere messo in dubbio. Uno dei principali canoni del metodo positivistico era che altro è il compito del giurista, altro quello del filosofo o del moralista o del politico. Per il giurista vale sempre il principio: dura lex sed lex. Il Gran Consiglio è diventato un istituto di diritto positivo? Il buon giurista si deve occupare del Gran Consiglio con la stessa indifferente neutralità con cui si era occupato durante l'età liberale della rappresentanza politica (anche se politicamente non era d'accordo). Il partito fascista diventa attraverso una norma giuridica, come quella sul Gran Consiglio, il motore dello stato? Il buon giurista dedicherà un dotto e accurato lavoro alla definizione della natura giuridica del PNF.

Tutt'altro fu il proposito con cui Trentin scrisse il suo trattato di diritto costituzionale: egli si propose di scrivere un'opera di critica politica attraverso la critica del diritto vigente. Egli non ebbe esitazione sin dalle prime pagine a definire lo stato fascista una "dittatura oligarchica» ${ }^{13}$ con una espressione che non si poteva trovare (e di fatto non si trova) in nessuno dei trattati di diritto pubblico scritti in Italia durante il ventennio, sotto il vigile occhio della censura. Naturalmente egli non si limitò a dare questa definizione ma cercò di esplicarla con un'analisi accurata e stringente dei nuovi testi legislativi, e con un confronto fra questi testi e la vecchia legislazione che veniva gradatamente soppiantata.

Così, mentre i trattati del tempo restano ormai sepolti nelle nostre biblioteche come opere morte e se vengono riletti la rilettura deve es-

${ }^{13}$ Ivi, p. 4. 
sere fatta con la massima cautela, con la stessa cautela con cui si leggono i documenti ufficiali, l'opera di Trentin rivive di vita nuova dopo più di mezzo secolo dalla pubblicazione e può essere letta con frutto da chiunque voglia approfondire lo studio dell'organizzazione dello stato durante il fascismo. Un giurista tedesco del secolo scorso è diventato famoso per aver scritto questa frase: «Bastano tre parole del legislatore e intere biblioteche di libri giuridici diventano carta da macero». Questa affermazione è giustissima se si riferisce alle biblioteche fatte di pedanteschi commentari, come quelli dei legisti della «Scuola dell'esegesi», spesso derisi ma più spesso imitati, che ispiravano il loro insegnamento alla massima «Stulta sapientia quae vult lege sapientior esse». Ma non è più valida se il commentario è ispirato a valori che trascendono il diritto posto, per esempio alla difesa dei diritti di libertà che sfida il tempo e le cattive stagioni della storia (che sono, come tutti sanno, molto più lunghe delle buone).

Quell'alta coscienza morale che fu Jemolo, giurista della buona e della cattiva stagione, nel libro autobiografico Gli anni di prova, descrivendo il progressivo ottundimento degli spiriti che un regime di polizia produce, si domanda se abbiano fatto bene o male la maggior parte dei professori, lui compreso, a prestare il giuramento imposto dal regime, e a restare in cattedra. Dopo aver esaminato scrupolosamente le pietose giustificazioni che allora vennero date e aver riconosciuto che "valevano poco", conclude: "Chi scelse la via buona furono quelli che, consci anche di ciò che significava il loro atteggiamento, nel Paese, fuori d'Italia, tra gli esuli, rinunciarono alla cattedra e testimoniarono» ${ }^{14}$. Silvio Trentin fu uno di questi rari testimoni.

14 A.C. Jemolo, Gli anni di prova, Neri Pozza, Vicenza 1969, p. 146. 



\section{LA CRISI EUROPEA E LO STATO FEDERALE NELL'OPERA DI SILVIO TRENTIN ${ }^{\star}$}

1. Quando parlai per la prima volta di Silvio Trentin, a Venezia, nel 1954 (dunque vent'anni fa), nel primo decennio della morte, la Resistenza era ancora vicina. Per quanto fosse già avvenuta quella che gli storici d'oggi chiamano la «restaurazione», il fascismo era dato per morto e sepolto. Si guardava con una certa ansia all'avvenire - non si dimentichi che fra le grandi potenze vincitrici era in corso la guerra fredda, e la lotta politica in Italia era sotto certi aspetti più aspra di quella di oggi -, ma tutti eravamo convinti di aver messo una pietra tombale sul recente passato. Ricordare allora l'opera e le gesta di un uomo come Silvio Trentin voleva dire esaltare il personaggio dell'intellettuale militante che aveva combattuto con il pensiero e con l'azione, con gli scritti e con le armi, durante il lungo esilio in Francia, e nei pochi mesi che gli furono concessi di vivere al suo ritorno in Italia, il mostro che aveva umiliato e atterrito, e poi distrutto e sconvolto l'Europa.

Oggi le cose sono cambiate. Ci siamo accorti in questi ultimi anni, e non solo in Italia, che il mostro non è morto, ma rinasce continuamente, se pure sotto altre forme, non appena il sistema democratico, che è l'unico metodo civile di convivenza che gli uomini abbiano inventato - un punto fermissimo nel pensiero di Trentin -, s'inceppa, e inceppandosi non riesce a dominare le tensioni troppo forti che si formano in una società in sviluppo. Per questo oggi rievocare il pensiero e l'opera di Trentin vuol dire ripercorrere una storia che non è ancora conclusa, ritornare sulle ragioni di una crisi storica - la grande crisi del primo dopoguerra che precipitò l'Italia nel fascismo e preparò le condizioni per la seconda ancora più terribile guerra mondiale - vuol dire insomma fare opera non soltanto di apologetica antifascista ma anche di critica storica.

Oggi più che mai gli storici s'interrogano con strumenti di analisi sempre più complessi, valendosi di una documentazione sempre più ricca, intorno alle cause e alla natura del fascismo. Perché nacque; perché in certi paesi piuttosto che in altri, o in certi paesi prima che in altri; quali

* La crisi europea e lo stato federale nell'opera di Silvio Trentin, in AAVV, Atti del Convegno di studio su Silvio Trentin, Jesolo, 20 aprile 1975, Neri Pozza, Vicenza 1976, pp. 109-123. 
diverse forme assunse nei diversi paesi; quali furono i ceti e le classi che lo sostennero; quali problemi affrontò, quali risolse o non risolse; quali furono le ragioni profonde che lo condussero a provocare una guerra micidiale in cui esso stesso sarebbe stato alla fine travolto ecc. Trentin visse personalmente, appassionatamente questa esperienza di crisi. La visse e la sofferse, prima con il lungo esilio, poi con la partecipazione alla resistenza; e vi meditò sopra lungamente. Gran parte delle sue opere, la parte più viva, quella che egli scrisse durante l'esilio in Francia oppure durante la guerra (prescindo in questa sede dalla sua opera di studioso di diritto), è una continua meditazione sulla crisi della civiltà europea, che l'avvento del fascismo in Italia e dei fascismi in Europa, aveva fatto esplodere. In questa meditazione si possono distinguere due aspetti o momenti: quello critico, che si risolve nell'esame delle condizioni dell'Europa dopo il fascismo e al momento dello scatenamento della guerra, e quello ricostruttivo attraverso cui egli cerca d'individuare le linee di tendenza di una trasformazione radicale che dovrà riportare l'Europa verso la pace e verso una rinnovata e più salda democrazia, e si lascia tentare di elaborare un progetto di nuovo assetto istituzionale al di là del grande ed apparentemente insanabile contrasto fra opposti sistemi economici e politici che ha insanguinato il mondo.

In questo mio discorso vorrei soffermarmi particolarmente su due punti: a) come Trentin vide e interpretò la crisi; b) quale fu il suo programma di ricostruzione dopo la crisi.

2. Trentin non ebbe alcun dubbio sul fatto che la prima grande manifestazione della crisi fosse stato il fascismo, contrariamente a tanti suoi coetanei, colleghi universitari, amici politici di cultura non mediocri, che pur essendo passati all'antifascismo militante, stettero per alcuni anni alla finestra, incerti verso qual parte spirasse il vento della storia. Fu uno dei tre professori universitari che rinunciarono alla cattedra (egli era professore d'istituzioni di diritto pubblico presso l'Istituto Superiore di scienze economiche e commerciali di Venezia dal 1923, dopo aver insegnato prima a Camerino poi a Macerata), quando il 24 dicembre $1925 \mathrm{fu}$ emanata una legge che toglieva ai professori universitari la prerogativa della inamovibilità. $\mathrm{Fu}$ dunque uno dei primi docenti della università italiana ad abbandonare l'insegnamento all'avvento del fascismo (uno dei primi, ed è inutile aggiungere, perché è storia nota, uno dei pochi). Fu anche uno dei primi antifascisti a scegliere l'esilio. Partì per la Francia alla fine di dicembre del 1925. Non ebbe alcun dubbio, dicevo, che il fascismo fosse il segno di una grande crisi, non solo politica, o economica, ma anche intellettuale, dell'Europa. Era una crisi passeggera o duratura? era una crisi soltanto italiana o anche europea? una crisi economico-politica o anche spirituale e morale? una crisi di una determinata società, come quella italiana sotto molti aspetti arretrata, o una crisi di civiltà? 
Col senno di poi sappiamo che il fascismo non fu soltanto un fenomeno passeggero e non fu soltanto italiano. Ma ciò che ora vediamo con tanta chiarezza non poteva essere altrettanto chiaro a coloro che avevano partecipato da protagonisti alla lotta politica finita nella dittatura.

In pochi anni, tra il 1928 e il 1931, Trentin scrisse alcune opere sul fascismo che rimangono, a parer mio, poi dei documenti più interessanti di quelle che possono oggi essere considerate le prime interpretazioni del fascismo, e che meriterebbero di essere maggiormente conosciute. Il primo libro è del 1928, ed è intitolato: L'aventure italienne. Légendes et réalités ${ }^{1}$. Sin dal titolo appare chiaro quale sia il giudizio che Trentin dà in un primo tempo sul fascismo. Il fascismo è un'avventura. Interpretare il fascismo come un'avventura significa che il nuovo regime, che ha distrutto lo stato liberale in Italia, e ha imposto una dittatura senza precedenti nel nostro paese, e sino al 1928, si badi, soltanto nel nostro paese, è un episodio abnorme, una specie di escrescenza emersa improvvisamente su un corpo sostanzialmente sano sebbene indebolito dalla guerra, un fatto atipico, patologico, eccezionale, la cui origine deve essere ricercata unicamente nella grande stanchezza provocata in un paese economicamente non robusto dai postumi di una malattia quasi mortale come la grande guerra europea. Nel 1929 scrive un vero e proprio trattato di diritto pubblico fascista, un'opera monumentale, ammirevole, dati i tempi e le circostanze in cui fu scritta, per la ampiezza della documentazione e per il rigore della denuncia: Les transformations récentes du droit public italien. De la Charte de Charles-Albert à la création de l'État fasciste ${ }^{2}$. Si tratta di una vera e propria storia costituzionale italiana in cui, mettendo a confronto il sistema vigente in Italia sino all'avvento del fascismo con quello instaurato in pochi anni di riforme costituzionali dal fascismo, mostra quello che la maggior parte dei professori di diritto costituzionale in Italia non volevano riconoscere o facevano finta di non vedere, e cioè che il fascismo aveva violato lo statuto e dato vita a un regime autoritario antitetico a quello cui bene o male si era ispirata la classe politica italiana dopo l'unità. Quando Trentin scriveva questo libro io frequentavo la facoltà giuridica di un'università italiana e apprendevo dai miei dotti maestri come equalmente il regime fascista fosse nient'altro che l'adattamento ai tempi mutati del vecchio statuto albertino diventato anacronistico e bisognoso di qualche rattoppo. Un libro come quello di Trentin ci avrebbe aperto gli occhi. Ma in Italia fu ignorato e per anni le nostre università conobbero soltanto $i$ testi ufficiali di diritto costituzionale fascista. Ma anche ora, quanti dei nostri giuristi lo conoscono? Mi accade ben poche volte di vederlo citato. Al contrario l'opera di Trentin fu ac-

\footnotetext{
1 Paris, Presses universitaires de France, 1928.

2 Giard, Paris 1929.
} 
colta allora in Francia con tutti gli onori: vi premise una ampia introduzione un noto giurista francese, Julien Bonnecase, il quale ne esaltava il rigore scientifico e non esitava ad affermare di sentire a ogni riga l'anima democratica dell'autore vibrare febbrilmente e profondamente davanti al parlamentarismo italiano svanito, alla costituzione sconvolta, alle libertà individuali calpestate e derise. Il libro era dedicato al figlio primogenito Giorgio con queste parole: «A mio figlio per insegnargli che la vita non merita di essere vissuta se non ha alla sua fonte la libertà, se è impotente a realizzare la libertà».

Per quel che riguarda l'interpretazione del fascismo l'opera più interessante è quella che apparve nel 1931, intitolata: Aux sources du fascisme ${ }^{3}$. Oggi è diventato quasi un luogo comune che sin dall'inizio vi siano state due interpretazioni opposte del fascismo: quella che si può dire gobettiana del "fascismo-rivelazione» (Gobetti aveva enunciato il suo famoso giudizio che il fascismo era l'autobiografia della nazione), e quella che si può chiamare crociana del «fascismo-parentesi» (Croce aveva detto più volte che il fascismo era una malattia grave ma passeggera). Senza abbracciare apertamente la tesi del fascismo-parentesi, Trentin combatte in questa opera con energia la tesi del fascismo-rivelazione. Sin dalle prime pagine polemizza contro la tesi che il fascismo «sia un prodotto caratteristico del genio del popolo italiano, come la consacrazione logica, inevitabile, fatale, delle sue tendenze, della sua educazione, delle sue tradizioni, della sua storia». Il fascismo è antidemocratico? Ma la democrazia, sostengono coloro che considerano il fascismo come una rivelazione di mali storici del paese non mai curati, non è mai esistita in Italia. Contro questa tesi Trentin sostiene, non senza influenza della Storia d'Italia del Croce (apparsa nel 1928), che prima dell'avvento del fascismo l'Italia era un paese liberale e democratico al pari dei paesi considerati più civili. Ne era una prova il fatto che il paese non si era dato mani e piedi al fascismo senza combattere. Anzi la lotta era stata tanto tenace che il fascismo aveva dovuto alla fine gettare la maschera, e mostrare il suo vero volto che era quello della dittatura e del dispotismo. Riprendendo la tesi, già sostenuta nel primo libro sull' avventura italiana", ribadiva il concetto che il fascismo non era stato affatto la prosecuzione, se pure aggravata, del malgoverno dell'età precedente, ma era stato l'effetto improvviso, imprevedibile, irripetibile, dell'esaurimento di tutte le energie nazionali, seguito ai sacrifici imposti prima dalla guerra e poi dalla pace. L'organismo ancor debole dello stato italiano era stato colto dalla crisi (che non era stata soltanto italiana) prima di aver raggiunto il suo pieno sviluppo: si era trattato quindi non di una crisi necessaria, quasi obbligata, ma di un momento di scoraggiamento, di sfiducia, che aveva permesso a un

3 Rivière, Paris 1931. 
gruppo di avventurieri senza scrupoli (ricordiamo il tema del «fascismoavventura») di conquistare il potere.

Vi è peraltro in questo libro in confronto con i precedenti un certo allargamento di visuale là dove l'autore spiega che coloro che approfittarono della situazione di debolezza organica dello stato furono i rappresentanti della "grande borghesia industriale». Egli è disposto a riconoscere che senza l'appoggio dell'alta borghesia industriale e finanziaria il fascismo non avrebbe mai potuto conquistare il potere e soprattutto non avrebbe mai potuto mantenerlo tanto a lungo dopo averlo conquistato. In questo modo il problema della nascita del fascismo viene collegato ad un'analisi, allora ancora da fare, sulla società italiana, e al violento scontro di classe che era esploso in tutta Europa e con particolare virulenza in Italia dopo la prima grande vittoria storica del proletariato guidato da Lenin nella rivoluzione russa. Inoltre ciò che Trentin colse subito con molta esattezza fu che il fascismo era l'antitesi radicale della democrazia, o, per riprendere il titolo di altro libro pubblicato l'anno prima, l'Antidémocratie $^{4}$. Ed espresse anche in quest'opera la convinzione - sulla quale mi piace richiamare in modo particolare la vostra attenzione nel momento che attraversiamo di "scricchiolamento" delle nostre istituzioni democratiche - che al di fuori della democrazia non c'è né dignità né salvezza né sviluppo morale e civile dei popoli. Testualmente: «Al di fuori delle forme democratiche ogni regime è fatalmente destinato a riprodurre integralmente le forme antiche e ben conosciute della tirannia politica». Da condannarsi, dunque, il fascismo proprio perché non rappresentava altro che «la realizzazione imprevista di una specie banale di tirannia politica che prende in prestito dai tipi classici che l'hanno preceduto nel corso della storia tutti i metodi e ogni sedicente ideologia». Precisò ulteriormente, in una vera e propria professione di fede, che la democrazia non può sopravvivere se non continuamente rinnovata. Riteneva che le condizioni per rinnovarla fossero le seguenti: a) lo stato deve opporsi al processo di concentrazione dei mezzi di produzione propria dell'attività capitalistica; b) deve allargare le autonomie locali contro la tendenza all'accentramento burocratico, caratteristico dello stato moderno; c) deve emanciparsi dalla concezione della lotta di classe perché questa concezione è incompatibile con la libertà.

3. Sino a questo momento Trentin è un democratico in senso generico, un democratico anzitutto, un democratico radicale che si contrappone al fascismo in quanto il fascismo è un regime dispotico, un improvviso ritorno (forse anche momentaneo) alla tirannia. Nel suo radicalismo democratico, che contrappone il potere dal basso al potere che scende

\footnotetext{
${ }^{4}$ Valois, Paris 1930.
} 
dall'alto, il principio delle autonomie al principio che domina lo stato accentrato e burocratico esaltato dal fascismo, Trentin può essere fatto rientrare in quel filone di democratismo radicale, che ha i suoi progenitori nelle correnti federalistiche del Risorgimento. L'idea autonomistica - intendo l'idea che la salvaguardia della libertà sia nel massimo sviluppo dell'autogoverno locale - rimane un punto fermo del suo pensiero. Se c'è un principio assolutamente inconciliabile col regime imposto dal fascismo e proprio questo: al potere popolare il fascismo ha contrapposto il potere del capo e dei suoi seguaci; al principio dell'eguaglianza, della maggior possibile eguaglianza, il principio della gerarchia, cioè dell'ordine che va dall'alto al basso; alla garanzia delle libertà individuali, la subordinazione degl'individui alla potenza dello stato, considerato il valore supremo di una collettività umana; alla partecipazione, l'obbedienza. Mentre la democrazia vuole trasformare la folla indistinta in popolo consapevole che si governa da sé, il fascismo ha ridotto il popolo che stava per prendere coscienza dei suoi diritti in folla che acclama il capo carismatico.

Peraltro sino a questo momento, come si è detto, Trentin ha fatto opera di critica del fascismo. Non ha ancora esposto il proprio programma politico per il dopo-fascismo. Il passaggio dal momento negativo al momento positivo o ricostruttivo, avviene con le due opere dottrinali (che sono anche le sue opere maggiori) di qualche anno più tardi: Riflessioni sulla crisi e sulla rivoluzione e La Crise du Droit et de l'Etat ${ }^{5}$. Tale passaggio è stato certamente influenzato dagli avvenimenti che caratterizzano la storia europea dopo il 1930. Mi pare che due siano soprattutto gli avvenimenti cruciali cui Trentin dà il massimo rilievo per cercare di determinare le tendenze e le linee di sviluppo del futuro dell'Europa e trarne suggerimenti per un programma d'azione: da un lato, la grande crisi economica, che rivela gli aspetti distruttivi delle società capitalistiche, dall'altro, sul versante opposto, l'emergere dello stato nato dalla prima rivoluzione socialista della storia, come potenza di prima grandezza. Due fatti contrastanti e che sembrano determinare una svolta nello sviluppo delle società umane sinora esistite: la civiltà capitalistica è in declino, la civiltà socialistica, annunziata da un secolo, ha avuto finalmente inizio e ha cominciato a spiegare la propria potenza. Intanto in Europa il fascismo non è più un fenomeno soltanto italiano, ma colpisce con maggiore o minore intensità tutti gli stati, e nel 1933, con l'avvento di Hitler al potere, penetra nel cuore della vecchia Europa. Ormai è diventato chiaro che il fascismo non è più soltanto un'avventura. Anzi si sta arrogando la pretesa di porsi come terza via oltre il capitalismo e il collettivismo. In realtà il fascismo non è che la soluzione disperata (destinata al più tra-

\footnotetext{
${ }^{5}$ Edizioni «Italia libera», Marsiglia 1933.

${ }^{6}$ Alcan, Paris 1935.
} 
gico fallimento) dei ceti che si vedono minacciati dalla «ribellione delle masse». In realtà è la vera e propria «ribellione delle masse», se si dà alla parola massa il senso spregiativo dei filosofi reazionari). Il fascismo è una terza via non nel senso positivo ma nel senso negativo, nel senso cioè di aver fatti propri, anziché gli aspetti positivi dei due sistemi contrapposti, gli aspetti negativi: del capitalismo il sistema della proprietà privata dei mezzi di produzione che perpetua e ingigantisce le diseguaglianze sociali, del socialismo, quale si veniva attuando nell'Unione sovietica, l'aspetto del regime politico autoritario, anti-liberale, antidemocratico, fondato sul monopolio del potere da parte di un partito unico che ha soppresso tutte le libertà civili e politiche pur sopravviventi, ancora, nonostante l'avanzata dei fascismi, nella maggior parte degli stati capitalistici.

Trentin si venne formando la convinzione che la soluzione dovesse essere cercata nella direzione diametralmente opposta. Sintesi, sì, ma positiva, non negativa. Non già - per esprimermi in una formula che può sembrare un po' semplificante ma ha il pregio della chiarezza - economia capitalistica e politica autoritaria (che caratterizzava il fascismo), ma, all'opposto, economia socialistica e politica democratica. Il punto di partenza rimane pur sempre il principio democratico, giacché la scelta ultima è sempre fra democrazia e dispotismo (dilemma che gli anni successivi e gli avvenimenti contemporanei hanno confermato, portando sempre nuovi argomenti alla tesi che là dove non c'è democrazia, c'è dispotismo, e tertium non datur). Ma qual è la via giusta per giungere alla democrazia? Il fatto che l'alta borghesia capitalistica e finanziaria avesse scelto il fascismo pur di salvare il sistema della proprietà privata dei mezzi di produzione, lo convince che il capitalismo sarebbe diventato a lungo andare sempre più incompatibile con la democrazia, e che pertanto in un'economia avviantesi verso la concentrazione delle imprese l'unico modo per distruggere l'immenso potere dei gruppi privati fosse la trasformazione dell'economia nella direzione del socialismo.

Mi par chiaro che Trentin sia arrivato al socialismo attraverso la democrazia, inteso il socialismo come l'unico sistema economico che avrebbe reso possibile una salda e duratura e integrale democrazia. Attraverso la democrazia e la critica del fascismo. Quando cominciò a combattere il fascismo, Trentin non era ancora, come si è detto, socialista. Era soltanto un democratico deluso, impaziente e indignato. Ma quando comincia a porsi il problema non più di criticare il fascismo ma di cercar di capire come sia possibile uscirne, e soprattutto come sia concepibile una società in cui il fascismo non sia più possibile, la democrazia da sola, come insieme di istituzioni che debbono servire a garantire la libertà dell'individuo nei riguardi del potere statale, e la partecipazione di ogni singolo al potere di tutti, non basta più. La democrazia è come un guscio; ma la bontà della noce non si può soltanto giudicare dal guscio. Bisogna andare a vedere che cosa c'è dentro. Fuor di metafora: dentro il sistema 
democratico politico che cosa ci dovrà essere in futuro: il capitalismo o il socialismo? Nelle opere teoriche, prima menzionate, a cominciare da quella del 1933, la risposta di Trentin non è dubbia: il socialismo. Tale risposta fu certamente suggerita dall'avanzata economica e politica dell'Unione sovietica dopo il primo piano quinquennale, cui faceva riscontro dall'altra parte la grande crisi economica del mondo capitalistico che aveva avuto inizio proprio negli Stati Uniti, punto di riferimento e centro d'irradiazione del capitalismo mondiale. Col dilagare del fascismo in Europa, Trentin si volge con simpatie sempre più forti e dichiarate verso l'Unione Sovietica, che gli sembra il baluardo più sicuro contro la fascistizzazione del mondo civile. Ma la sua accettazione del sistema sovietico è condizionata: la condizione è rappresentata dalla mancata democratizzazione. Vi è pur sempre una soglia che un democratico convinto non può superare, e questa soglia è la garanzia delle libertà civili e politiche. Pur nella fiducia nei riguardi dell'Unione Sovietica, nella capacità che riconosce ad essa di ergersi come barriera insuperabile contro il fascismo, Trentin resta fedele all'ideale dell'autonomia. Buon governo ancora una volta è soltanto quello che permette di realizzare nella più ampia misura possibile l'autogoverno. Di democrazia parlano tanto gli Stati Uniti a nome del liberalismo, quanto l'Unione Sovietica a nome del socialismo. Ma la realtà di fatto in entrambi i paesi è ben diversa. La soluzione dunque non potrà essere che al di là del capitalismo e al di là del socialismo (del socialismo, almeno, quale si è imposto nell’Unione Sovietica).

4. Nonostante tutto, l'atteggiamento di Trentin nei riguardi dell'Unione Sovietica è diverso da quello nei riguardi degli Stati Uniti. Verso il capitalismo è di rifiuto totale. Verso il socialismo sovietico è di accettazione, come si è detto, condizionata. Egli ritiene storicamente inoppugnabile e praticamente valido il contributo dato dall'Unione Sovietica alla lotta e alla sconfitta del capitalismo. Per combattere i critici della patria del socialismo, che erano numerosi anche nelle file del movimento Giustizia e Libertà cui egli apparteneva, se pure in posizione personale, egli scrive la prima opera dottrinale, citata, Riflessioni sulla crisi e sulla rivoluzione. La rivoluzione russa ha dimostrato, a suo giudizio, la capacità istituzionale dello stato a gestire l'economia. Non è vero che il miglior sistema economico sia quello capitalistico: la grande crisi ha dimostrato che il capitalismo non è più in grado di dominare le forze che ha scatenato. D'altro canto, è vero che l'Unione sovietica ha instaurato il sistema socialistico di produzione ma non è riuscito a conciliarlo col metodo della libertà. Riecheggiando Gobetti, che non cita e forse non conosce, ma verso le idee del quale rivela un'affinità ideale, si proclama «rivoluzionario liberale». Alla fine dell'opera scrive con una certa solennità: l'abisso che ci separa dalla Russia si chiama «libertà». All'inizio aveva proclamato la sua fede scrivendo: «È vano pretendere di poter transigere impunemente 
con il metodo della libertà, perché le esigenze di questa sono e restano categoriche e irriducibili».

Nell'ambito del movimento Giustizia e Libertà il tentativo puramente teorico di andare al di là di liberalismo e socialismo non era nuovo. Anzi era in un certo senso il carattere peculiare del movimento. Carlo Rosselli aveva esposto qualche anno prima (1928) una teoria del «socialismo liberale" che era diventata il programma del movimento, e sarà in seguito pure il programma del Partito d'azione, in cui confluirà il movimento sotto certi aspetti analogo, se pure nato in ambiente diverso, tra intellettuali italiani della giovane generazione senza radici nell'esperienza politica prefascista, del liberalsocialismo. Ma nonostante la convergenza verso l'idea del superamento delle due opposte ideologie del liberalismo e del socialismo, la dottrina di Trentin è diversa, sia nei presupposti teorici, sia nel giudizio storico e nelle conclusioni politiche, da quella di Rosselli. Rispetto all'evento capitale nella storia del socialismo, la rivoluzione sovietica, la dottrina di Rosselli si presenta come un tentativo di superamento; quella di Trentin invece rappresenta piuttosto il tentativo di accettarla nei suoi aspetti positivi e di trasformarla in rivoluzione democratica. Per i socialisti liberali la rivoluzione democratica dovrà essere una nuova rivoluzione; per Trentin la rivoluzione democratica dovrà coincidere in Italia con la rivolta contro il fascismo e dovrà essere l'inveramento della rivoluzione sovietica che ha realizzato il socialismo ma non la democrazia. Dalla diversità dei giudizi storici discende anche una differenza nel programma politico: più prammatico quello rosselliano, più dottrinario quello di Trentin. Mentre Trentin accetta incondizionatamente il sistema economico socialistico, nel suo significato autentico di sistema fondato sulla collettivizzazione integrale (da ottenersi, aggiunge Trentin, anche rivoluzionariamente), il programma di Giustizia e Libertà, che sarà poi riproposto dal Partito d'azione, prevede piuttosto la spartizione dell'economia nel settore pubblico e nel settore privato, che è programma più laburista che non socialista. La verità è che i socialisti liberali e i liberalsocialisti vogliono, sì, diminuire la potenza dei gruppi privati che spadroneggiano nella sfera economica e influenzano il potere politico, ma guardano con minore diffidenza allo strapotere dello stato. Non è che anche Trentin non diffidi dello stato: la sua battaglia autonomistica è una battaglia antistatalistica. Ma in quanto autonomista egli ha in mente una nuova forma di stato, che non è né quello liberaldemocratico, che pende dalla parte dell'individuo e rischia continuamente di precipitare nell'anarchia (con la conseguenza di far nascere il fascismo come reazione), né quello sovietico, che pende dalla parte dell'autorità pubblica e rischia di finire nel totalitarismo. La nuova forma di stato che egli ha in mente è quella dello stato federale. Dunque, se rispetto al sistema economico è socialista, rispetto al sistema politico è federalista. 
Il tema del federalismo, destinato a diventare il tema centrale del progetto di ricostruzione del nuovo stato, appare ormai tutto spiegato nella seconda opera dottrinaria, La Crise du Droit et de l'État, che è del 1935. Ritengo che questa sia una delle opere più importanti di Trentin, anche se la meno nota. Il diritto e lo stato di cui, secondo Trentin, è ormai aperta la crisi inarrestabile, sono il diritto e lo stato derivati dalla Rivoluzione francese: nati per proteggere l'individuo hanno in realtà abbandonato $i$ deboli nelle mani dei forti. Di fatto lo stato accentrato - lo stato che egli chiama «monocentrico» - è passato pari pari dalle monarchie assolute ai moderni stati rappresentativi, che pur pretendono di essere democratici. Dal momento che la crisi del vecchio diritto e del vecchio stato è aperta, occorre inventare un nuovo diritto e un nuovo stato che siano in grado di salvaguardare la libertà reale, la libertà di tutti, e non solo di coloro che possiedono, e insieme di controllare l'economia in modo che non diventi uno strumento di sopraffazione. A questo scopo s'impongono due direttive: a) l'emancipazione dell'individuo attraverso la soppressione del privilegio economico; b) la rigenerazione delle basi istituzionali dello stato attraverso il federalismo. In opposizione allo stato monocentrico dei grandi paesi europei, come la Francia e l'Italia (che dalla Francia l'ha ereditato), lo stato federale è lo stato policentrico, cioè è lo stato che rispecchia fedelmente nelle sue strutture la pluralità dei gruppi sociali in cui gl'individui operano, producono e consumano, e non solo non li soffoca ma ne tutela l'esistenza e l'attività, e ne promuove il libero sviluppo.

5. Allo specifico tema del federalismo e dello stato federale Trentin dedica due opere successive e in un certo senso conclusive: una di carattere storico, Stato, nazione, federalismo, scritta nel $1940 \mathrm{ma}$ apparsa postuma a cura del Partito d'azione nel 19457; l'altra di carattere teorico, Libérer et Fédérer, che ha per sottotitolo Dare uno scopo e un senso alla rivoluzione europea in corso di gestazione, scritta probabilmente negli stessi anni (all'inizio della guerra), pubblicata nel volume intitolato Scritti inediti. Testimonianze e studi ${ }^{8}$. La prima contiene soprattutto una critica storica dello stato moderno, il quale, non importa se monarchico o democratico, se fondato sulla sovranità del principe o sulla sovranità del popolo, si è venuto formando attraverso una progressiva concentrazione del potere, che ha soffocato via via la vita dei corpi intermedi e degli enti locali, e ha dato origine a un apparato burocratico sempre più gigantesco, che si oppone allo sviluppo dell'autogoverno. La critica storica precede la progettazione politica: ciò che Trentin vuole dimostrare attraverso l'opera storica è che al punto in cui è arrivato il processo di concentrazione del potere

${ }^{7}$ La Fiaccola, Milano 1945.

8 A cura del Centro Studi Piero Gobetti, Guanda, Parma 1972. 
statale, se si vuol salvare la libertà individuale e la dialettica dei gruppi, non c'è altra soluzione che quella della disarticolazione della compagine statale e della sua ricostruzione su basi federali.

Il saggio Libérer et Fédérer è un vero e proprio programma politico, che si può dividere in tre punti fondamentali: a) il sistema capitalistico, nonostante le correzioni e i ritocchi cui viene continuamente sottoposto è impotente a fronteggiare la crisi della società contemporanea; b) l'evoluzione della tecnica della produzione richiede perentoriamente una soluzione globale che non può essere data se non da un'economia diretta $\mathrm{e}$ pianificata; c) solo la struttura federalistica dello stato impedisce allo stato collettivista di degenerare nello stato totalitario, cioè rende possibile la conciliazione della collettivizzazione economica con la libertà politica. Resta a dire che per struttura «federale» dello stato Trentin intende una struttura piramidale del potere in cui il flusso del potere non va dal vertice alla base ma procede al contrario dalla base al vertice, ha una direzione non discendente ma ascendente. La società organizzata è sempre una piramide: si tratta di vedere se è il vertice che comanda alla base o la base al vertice. Nello stato unitario il flusso del potere parte dal vertice; nello stato federale, dalla base. Trentin è perfettamente consapevole che questo suo progetto non è affatto nuovo: è la ripresa e la rivendicazione di quell'idea dello stato dei "consigli», che la rivoluzione russa aveva iscritto nel suo programma e che non ha mai attuato, sostituendo allo stato democratico dei molti consigli lo stato autocratico del partito unico.

Non ho bisogno di sottolineare l'importanza e anche l'attualità di questa proposta: l'idea di uno stato dei «consigli» è, com'è ben noto, un'idea ricorrente della democrazia socialista, che rinasce ad ogni sussulto delle società organizzate e dirette dai partiti comunisti (d'osservanza leninista) al potere, e si pone come alternativa - come unica alternativa possibile allo stato rappresentativo, considerato come espressione ormai arretrata e degenere, del pensiero politico liberale e borghese. Lo stato dei consigli, invocato da Lenin negli anni della rivoluzione in atto, rappresenta la trasformazione della democrazia parziale, che sarebbe la democrazia realizzabile nello stato della borghesia, in democrazia integrale. Trentin avrebbe potuto richiamarsi anche a Marx, che esalta la Comune di Parigi come l'embrione di un nuovo stato, e lo chiama, con una formula destinata ad aver fortuna, l'«autogoverno dei produttori». Più correttamente si richiama alla tradizione del socialismo libertario e pluralista che ha il suo maggior rappresentante in Proudhon. Essendo Trentin molto parco nei riferimenti agli autori da cui si possa desumere quali siano state le sue fonti, tanto più significative sono le pagine che nel saggio, Libérer et Fédérer, dedica a Proudhon, cui non risparmia critiche e accuse di astrattezza e di semplicismo, ma cui ciononostante riconosce il merito di aver avuto «illuminazioni e profezie sorprendenti, soprattutto in relazione alla decifrazione delle cause profonde della crisi della società contemporanea 
e alla scoperta delle leggi generali il cui riconoscimento soltanto potrebbe permettere di sormontarla»?.

Per sfuggire al rimprovero che egli stesso aveva mosso a Proudhon di non essersi mai preoccupato di dare indicazioni più precise circa il proclamato federalismo (scambiato a torto con l'anarchismo), Trentin scrisse, quando era già cominciata la resistenza armata contro il nazismo e il momento della liberazione appariva prossimo, due abbozzi di costituzione di uno stato federale, uno per il nuovo stato francese, l'altro per il nuovo stato italiano, in cui traccia le linee maestre di quella nuova forma di stato che avrebbe dovuto permettere il funzionamento di un sistema economico socialista attraverso l'ordinamento di uno stato democratico e garante delle libertà fondamentali.

Il cardine di questo nuovo ordinamento è l'art. 9 del progetto di costituzione della Francia (ripreso nel preambolo della costituzione per l'Italia), nel quale si afferma che la costituzione della repubblica federale è fondata sui cinque grandi principi del "governo popolare diretto", dell'«autonomia istituzionale», della «libertà della persona», della "proprietà comunitaria», e della "giustizia sociale». Il confronto fra questi abbozzi di costituzione e le costituzioni di tipo più tradizionale che saranno poi emanate in vari stati europei, come la Francia, l'Italia e la Germania, dopo la liberazione, sarebbe molto istruttivo. Le costituzioni post-belliche che più si avvicinano ai progetti federalistici di Trentin sono quelle jugoslave, ispirate al principio della democrazia dei consigli, e della rappresentanza per gradi successivi, dagli enti locali sino al parlamento nazionale.

6. "Liberare e federare» diventò per Trentin una parola d'ordine. E fu anche il titolo che egli diede al movimento di resistenza da lui fondato insieme con alcuni suoi amici nel Sud-Ovest della Francia nel 1942, dopo aver assistito impotente e corrucciato alla sconfitta dell'esercito francese e alla fine ingloriosa della Terza Repubblica (sulla quale scrisse di getto un saggio lucido ed ispirato, L'abdicazione della Francia o la fine di un mondo, che ha un sottotitolo che ne rivela lo stato d'animo, Note di un sopravvissuto, dove la disfatta della Francia, improvvisa ma non senza profonde cause morali e politiche, è vista come la fine dolorosa di una civiltà, destinata a soccombere per lasciare il posto a un profondo rinnovamento). Il gruppo di resistenti cui diede vita ebbe pure un suo giornale, intitolato anch'esso, «Libérer et Fédérer». Di questo giornale Trentin fu promotore, organizzatore e collaboratore. L'antecedente storico del movimento, come ha raccontato distesamente Hans Werner Tobler, era stato il «réseau» fondato dal giovane germanista, Pierre Bertaux. Arrestati quasi tutti i componenti di questo primo raggruppamento alla

${ }^{9}$ Ivi, p. 263. 
fine del 1941, la direzione del movimento era passata a un giovane lettore, Gilbert Zaskas, in rapporto strettissimo col Trentin. Confrontato con gli altri movimenti della resistenza francese, il gruppo Libérer et fédérer ha una sua caratteristica più volte notata dagli storici: non è solo un movimento per la lotta armata - fu una delle organizzazioni della SOE (Special Operations Executive), cioè del comando «operazioni speciali», specie sabotaggi -; ma ebbe anche un suo specifico contenuto dottrinale, una sua ideologia, che si distingue dal generico antifascismo e dall'ancor più generico rivoluzionarismo negativo, per un suo tentativo cosciente di elaborare un programma di ricostruzione sociale e politica a liberazione avvenuta. Questo programma consisteva, inutile il ripeterlo, nella trasformazione dello stato accentrato in uno stato federale. Le idee del movimento, che sono le idee già esposte di Trentin, sono contenute in un numero speciale del giornale dedicato a I tre problemi fondamentali della libertà nel mondo moderno.

Trentin aveva combattuto in Francia perché non aveva potuto combattere in Italia. Ma caduto il regime fascista, non esitò a tornare in patria. Tornò come tanti esuli per partecipare alla guerra di liberazione nazionale, non appena si ricostitui il fronte antifascista. Arrivò fra noi nel settembre 1943. Morì troppo presto nel marzo del 1944 dopo un breve arresto per poter dare alla lotta il contributo che avrebbe voluto e di cui noi, più giovani che allora lo incontrammo, avremmo fatto tesoro. Resta come documento della sua opera dedicata alla resistenza italiana il progetto di costituzione, che ho menzionato poco fa, scritto a nome del gruppo regionale veneto del Partito d'Azione (di cui fu, in quei pochi mesi, una guida sicura e autorevole). Il progetto, intitolato Piano tendente a delineare la figura costituzionale dell'Italia al termine della rivoluzione federalistica in corso di sviluppo, comincia con queste linee: "L'Italia è una repubblica federale e rivendica, in questa sua qualità, la dignità e il titolo di membro fondatore della Repubblica europea. Essa colloca in testa alla sua Carta ed erige a criterio supremo per la legittimazione del funzionamento dello stato i grandi principi della libertà della persona, dell'autonomia istituzionale, della proprietà collettiva e della giustizia sociale». Era un'Italia nuova quella che egli aveva in mente, un'Italia purificata attraverso la lotta che stava allora per cominciare. Un'Italia libera e giusta non solo verso i propri cittadini ma anche verso gli altri popoli. Un'Italia democratica quale avevano vagheggiato gli scrittori radicali del Risorgimento, i primi socialisti, i gruppi di antifascisti in esilio che avevano dato vita al movimento di Giustizia e Libertà. Vagheggiata ma ben lontana dall'essere diventata anche nei momenti più alti una vivente realtà. Occorreva che l'abiezione avesse toccato il fondo con il fascismo e con la perversa alleanza del fascismo col nazismo perché il paese si destasse a nuova vita e si cingesse la testa non dell'elmo di Scipio, come suonava una canzone del Risorgimento diventata inno ufficiale della Repubblica italiana, ma 
del berretto frigio, simbolo della rivoluzione popolare. E facesse proprio il motto che Giustizia e Libertà si era dato: «Insorgere per risorgere».

Silvio Trentin era nato nella vostra città l'11 novembre 1885. Fu sempre fedele nel ricordo e nella speranza alla città natale. Nel volume già ricordato dei suoi scritti inediti c'è una bella fotografia che lo ritrae insieme con la famiglia, festante, in mezzo a un folto gruppo di concittadini il 6 settembre 1943, il giorno del suo ritorno in patria. Sono passati da quel giorno più di quarant'anni. Chi sa che sia ancora vivo qualcuno che si riconosce nelle persone che lo circondano in atto di giubilo e di omaggio. Potrebbero raccontarci quali sentimenti li animarono in quel momento, quali speranze li agitavano, che cosa abbia significato per loro l'improvviso e felice ritorno alla libertà, rappresentato simbolicamente dal ritorno in patria dell'esule. Sono rimaste vive quelle speranze? Lascio la domanda volutamente senza risposta. Ciò che importa è che sia rimasto vivo l'impegno. Perché solo così Silvio Trentin è veramente di nuovo fra voi. Per riaccoglierlo oggi, come l'accoglieste quarant'anni fa, occorre respingere ogni tentazione di distruggere quello che la Resistenza ha faticosamente costruito. Occorre allargare, rafforzare, perfezionare, come egli avrebbe voluto, la nostra democrazia. Vi è un solo modo serio per commemorare Silvio Trentin: restare fedeli agli ideali per i quali egli e gli uomini di fede come lui hanno combattuto. Non costringerlo ad andare un'altra volta in esilio. 


\section{L'OPERA DI SILVIO TRENTIN ${ }^{\star}$}

Con una eccezionale precisione e puntualità, di cui va data lode ai promotori, ai curatori, e in modo particolare all'editore, da quando la nobile e non facile impresa di pubblicare le opere di Silvio Trentin è stata progettata, ogni anno è uscito un volume:

1983: l'opera di diritto costituzionale, a cura di Alessandro Pizzorusso; 1984: gli scritti giuridici e amministrativi prima dell'esilio, a cura di Moreno Guerrato; 1985: gli scritti antifascisti dell'esilio, a cura di Giannantonio Paladini;1986: gli scritti federalistici vengono oggi presentati in questa solenne cerimonia, promossa dal comune di Venezia; col quinto volume curato dal prof. Ventura, in cinque anni il progetto ambizioso in questi tempi non fausti per l'editoria sarà compiutamente attuato, al di là delle nostre più ottimistiche aspettative ${ }^{1}$.

Avremo così dato vita e anima a un corpus coerente e organico di circa 2000 pagine di scritti giuridici, politici, storici, composti in un arco di tempo di 35 anni dal 1916 al 1943 per la maggior parte negli anni tormentati dell'esilio.

Si badi, sono "opere scelte", come si legge chiaramente nell'intestazione di ogni volume. Non tutti gli scritti sono stati raccolti. Non tutti quelli raccolti sono stati riprodotti per intero. Basti dire che della grande opera del periodo della maturità, La Crise du Droit et de l'État, è stato riproposto un solo capitolo.

Si tratta a ogni modo di una scelta amplissima, oculata, ben calcolata e dosata, tale che nulla è stato sacrificato di ciò che possa permettere non solo al lettore comune ma allo studioso una conoscenza compiuta del pensiero di Silvio Trentin.

* L'opera di Silvio Trentin, discorso pronunciato a Venezia il 6 dicembre 1986, in occasione della presentazione del IV volume delle "Opere Scelte di Silvio Trentin", Federalismo e libertà. Scritti teorici 1935-1943, Marsilio, Venezia 1987, inedito. Le sottolineature sono di Norberto Bobbio.

${ }^{1}$ Cfr. la Bibliografia di e su Silvio Trentin in appendice, a cui si rimanda per le informazioni bibliografiche relative ai testi citati nel discorso di Bobbio. 
Ho parlato di un corpus coerente ed organico. Pur nel passare degli anni e del mutare delle vicende storiche (e quali vicende!), la prima guerra mondiale, la crisi sociale e politica post-bellica, il fascismo, la seconda guerra mondiale, la Resistenza, il pensiero di Trentin è ispirato costantemente a un'idea fondamentale, all'idea del potere dal basso. Come avrebbe detto uno degli scrittori del nostro Risorgimento che gli era caro, Carlo Cattaneo, per conservare la libertà i popoli debbono tenerci sopra le mani.

Una tesi di questo genere si regge, come il mondo sulle spalle di Atlante, su un principio morale fondamentale, uno di quei principi - o postulati etici direbbe un filosofo - che hanno valore assoluto che non si discute e sul quale non è possibile alcuna transazione: il valore della dignità dell'uomo.

L'idea del potere dal basso - che è poi il caposaldo di ogni possibile governo democratico - ispira nei primi anni la battaglia politica immediata per l'ampliamento delle autonomie amministrative, negli ultimi la battaglia dottrinale e pratica nel movimento di resistenza "Libérer et Fédérer" per lo stato federale. Negli anni di mezzo, che corrispondono al periodo dell'esilio francese, scorre la critica senza tregua, attraverso scritti storici, giuridici, teorici e di polemica quotidiana, della concezione autoritaria dello stato, che ha trovato nel fascismo italiano la sua prima e contagiosa attuazione pratica.

Che nei primi anni egli pur battendosi in difesa delle autonomie locali continui a sostenere la necessità dello stato unitario, e nell'ultima parte della vita giunga alla convinzione che l'unica forma possibile di stato dell'avvenire, sia lo stato federale, è segno non tanto di un mutamento nell'orientamento del proprio pensiero quanto di un adattamento di una dottrina sin dall'inizio autonomista al mutamento della situazione politica generale, che ha rivelato, attraverso l'avvento degli stati totalitari, la crisi profonda, irreversibile dello stato unitario.

La crisi di cui si parla è la crisi dello stato unitario che conduce Trentin alla conclusione che lo stato del futuro non può essere che un ordinamento delle autonomie (un ordinamento che presuppone le autonomie e che deve rispettarle e garantirne l'esistenza). Oggi useremmo l'espressione ormai entrata anche nell'uso giornalistico: pluralismo. La concezione che Trentin ha del futuro stato, che deve sorgere dalle ceneri dello stato autoritario, è una concezione pluralistica, la sola compatibile con la forma di governo democratico: "Lo stato non può organizzarsi se non organizzando la società, e la società a sua volta non può essere organizzata secondo il diritto che conformando la sua organizzazione all'imperativo che pone il valore supremo nell'autonomia della persona e in quello degli ordini richiesto dall'esercizio dei suoi attributi».

Autonomia delle persone, ma anche degli ordini. Ormai dalla concezione roussoiana dello stato che presuppone l'autonomia solo degli in- 
dividui e non ricorre, anzi rifiuta gli ordini, si è passati alla concezione più complessa, effetto della trasformazione storica delle società più evolute, della società in cui soggetti politici non sono soltanto gli individui ma anche i gruppi organizzati, di cui una costituzione democratica, non può non tener conto.

L'art. 2 della nostra Costituzione - come tutti sanno - rispecchia questa considerazione della società hobbesiana, più realistica di quella astratta roussoiana, introducendo accanto al riconoscimento dei diritti inviolabili dell'uomo come singolo anche quelli delle formazioni sociali.

Degli scritti più propriamente federalistici il primo è il volume Stato Nazione Federalismo, concepito intorno al 1940, pubblicato per la prima volta presso la casa editrice la Fiaccola di Milano nel 1945, come n. 1 d'una biblioteca di cultura politica con prefazione di Mario Dal Pra.

Era una iniziativa del PdA, cui Trentin aveva aderito tornando in Italia dall'estero, pur contestando in una famosa lettera del 23 ottobre ' 43 a Lussu la linea politica di fondo. In realtà il PdA non aveva in realtà una precisa linea politica - ne aveva molte -, il che lo portò a una rapida fine. (Ma forse il giudizio severissimo di Trentin «una raccolta di sbandati» non può essere presa alla lettera). Durante gli anni della guerra di liberazione erano stati pubblicati, in clandestinità, $\mathrm{i}$ "Quaderni dell'Italia libera», in cui erano stati svolti da autori che si coprivano dietro uno pseudonimo i principali problemi della futura ricostruzione. La loro lettura sarebbe molto istruttiva per far conoscere la distanza fra i progetti che si fanno a tavolino e la realtà, ma anche per dare una rappresentazione viva, concreta, convincente, degli eterni problemi non risolti nel nostro paese.

La Biblioteca di cultura politica che ebbe inizio con la pubblicazione dell'opera di Trentin avrebbe dovuto continuare idealmente la serie dei «Quaderni», ma si fermò al primo volume. Con la liberazione sorsero varie case editrici ormai senza vincoli di clandestinità, e il PdA, che era un partito di intellettuali, anzi il partito degli intellettuali, non ebbe che l'imbarazzo della scelta per far conoscere le proprie idee. Penso ad esempio alla collana "Giustizia e libertà» delle Edizioni U di Roma-Firenze-Milano, diretta da Aldo Garosci che vi pubblicò la traduzione di Socialismo liberale di Carlo Rosselli (n. 2) e Vita di Carlo Rosselli. Il primo volume della collana è stato La Catena di Emilio Lussu, che fu di Trentin amico affettuosissimo e di Trentin scrittore fervido ammiratore. Egli disse di lui: è «un socialista non marxista, un socialista che spinge le sue tesi alle audacie più estreme, un socialista massimalista che dal comunismo è separato solo da una visione differente della civiltà e da una concezione immanentista della libertà». Furono pubblicati in questa collana libri di Leo Valiani, Aldo Capitini, Nello Rosselli, Barbara Allason.

Non vorrei sbagliare: per vedere le prime opere di Trentin pubblicate dopo la Liberazione bisogna arrivare al 1972 quando apparvero gli Scritti inediti, a cura di Paolo Gobetti e per iniziativa del Centro studi Piero 
Gobetti di Torino. Nel 1975 uscì con l'introduzione di Enzo Santarelli, presso gli Editori Riuniti, la traduzione di un libro dell'esilio, scritto alla fine del 1937, Dieci anni di fascismo, promosso dal Centro di ricerca Silvio Trentin di Iesolo, diretto con passione e costanza che non sono mai venute meno dal dottor Raffaello Zannoner.

Al volume Stato Nazione Federalismo segue l'ampio saggio di carattere prevalentemente teorico, Libérer et Fédérer, scritto in francese intorno al 1942, rimasto inedito sino al 1972. La miglior prova della scarsa diffusione delle opere di Trentin, dovuta alla sua morte precoce, è la vicenda di questo scritto. Che Trentin intendesse diffonderlo subito può essere provato dal fatto che egli ne diede una copia perché lo traducesse ad Antonio Giuriolo, vicentino, letterato, scrittore, studioso di letteratura francese, che fu ucciso dai tedeschi in un breve conflitto a fuoco sull'Appennino tosco-emiliano il 12 dicembre 1944. Giuriolo gli aveva fatto visita due volte a San Donà di Piave ${ }^{2}$. La traduzione fu fatta dallo stesso Giuriolo e dall'amico Nino Perego per incarico del CLN veneto, ma allora non vide la luce.

Nel bel libro di un giovane Antonio Trentin su Antonio Giuriolo viene raccontato l'incontro di questi con Trentin, e si fa l'ipotesi che le idee di Trentin abbiano avuto una forte influenza sul letterato-combattente, che proveniva, come gran parte dei giovani intellettuali azionisti, più dalla tradizione di pensiero liberale (impersonato da Croce) che non da quella socialista.

L'interesse storico del saggio sta nell'essere un tentativo di sintesi, allora frequente, tra i due opposti sistemi, quello capitalistico occidentale e quello collettivistico sovietico, di cui gli spiriti più inquieti, più aperti al dubbio e alla critica, erano profondamente insoddisfatti. Era opinione diffusa che al crollo dei regimi fascisti, che erano stati per un verso antiliberali (in politica) e antisocialisti (in economia), la storia dovesse percorrere una via completamente nuova, che non avrebbe più dovuto essere di antitesi tra i due sistemi, tra un eccesso di libertà che aveva approfondito le disuguaglianze tra coloro che hanno e coloro che non hanno, e un eccesso di eguaglianza che era stato ottenuto attraverso il soffocamento della libertà individuale, ma dovesse trovare una via completamente nuova, al di là delle due esigenze, che scontratesi in una lotta senza quartiere avevano generato il mostro dei regimi totalitari fascisti.

La sintesi cui mirava Trentin era più originale, e anche più audace, di quella tentata dal socialismo liberale di Rosselli (o dal liberal-socialismo di Calogero e Capitini). La sintesi di Rosselli (così come quella di Calogero) era in realtà una conciliazione pratica delle due esigenze, e teoricamente un superamento che spesso appariva soltanto nominale. La sintesi

${ }^{2}$ [In realtà a casa Nardari a Treviso]. 
di Trentin era una composizione che derivava dalla convinzione che il collettivismo economico aveva ormai combattuto efficacemente il capitalismo, ma non aveva mantenuto fede, nella degenerazione politica del regime staliniano, agli ideali di libertà da cui era stato ispirato. Rispetto al socialismo liberale di Rosselli, il procedimento di Trentin era opposto. Per i liberal-socialisti, che partivano dalla tradizione del pensiero liberale, si trattava di socializzare il liberalismo, per Trentin, che partiva dall'esigenza di superare il capitalismo, si trattava di liberalizzare il collettivismo. La via per liberalizzarlo era lo stato federale, uno stato cioè che doveva capovolgere completamente la tendenza all'accentramento sempre più rigido, quale si era manifestato nell'Unione Sovietica. Il collettivismo economico deve liberare l'individuo come produttore dalla schiavitù del potere economico; il federalismo deve liberare l'individuo come cittadino dalla schiavitù del potere politico.

Il federalismo accetta lo stato contro la negazione che dello stato fanno gli anarchici, ma rifiuta lo stato accentratore, e lo trasforma da ordinamento statale in un ordine degli ordini: "Lo stato di domani, se vuole realizzare un progresso rispetto allo stato di oggi, sul piano dell'affrancamento dell'individuo e della salvaguardia della dignità della persona, non può essere che federalista».

Non sarà mai sottolineata a sufficienza l'importanza che ebbe l'idea federalistica nei movimenti della Resistenza. Va detto subito però che delle due facce del federalismo, l'esterna e l'interna, la faccia esterna rivolta all'ordine internazionale, in quanto superamento della sovranità assoluta dei singoli stati verso la costituzione di uno stato di stati (come era avvenuto due secoli prima nei rapporti tra le 13 colonie dell'America del Nord, dopo la vittoriosa guerra di liberazione dal giogo coloniale), era stata quella di gran lunga prevalente nei movimenti della Resistenza, dai quali erano scaturiti vari progetti di Stati Uniti d'Europa, tra i quali quello di Ernesto Rossi, Colorni e Spinelli, detto Manifesto di Ventotene: il progetto degli Stati Uniti d'Europa era inserito nel programma del Partito d'Azione. Nel programma politico di Trentin è invece prevalente l'interesse per l'aspetto interno: ciò che gli preme non è tanto l'aggregazione dei singoli stati in una unità più vasta quanto la disgregazione $\mathrm{o}$ disarticolazione d'ogni singolo stato unitario in membra relativamente autonome rispetto al tutto. Detto altrimenti Trentin considerava il problema centrale del tempo non tanto l'anarchia internazionale quanto il dispotismo interno. Naturalmente la distruzione dei regimi dispotici avrebbe facilitato il superamento dell'anarchia internazionale.

Il problema della libertà e della pace sono strettamente connessi per una ragione semplicissima: un regime libero è meno bellicoso e guerrafondaio che uno dispotico. Che cosa diceva Kant in quell'aureo libretto che è La pace perpetua? Se si vuole la pace internazionale, la prima condizione è che ogni stato assuma «forma repubblicana», vale a dire una for- 
ma di governo tale che il volere del sovrano fosse controllato dal popolo. Non sono sicuro che la proposta di Kant sia infallibile, anche perché sinora una società internazionale in cui tutti i governi siano alla maniera di Kant repubblicani, non è mai esistita. Ma è un fatto che i vari progetti di Stati Uniti d'Europa che fiorirono in quegli anni facevano parte integrante dei movimenti democratici più radicali. All'attuazione dell'ideale federalistico, inteso come disarticolarsi dello stato unitario in uno stato federale o ordine delle autonomie, Trentin pose mano non dimenticando di essere stato un giurista, scrivendo un completo progetto di costituzione, anzi due, uno per la Francia e uno per l'Italia. Non ricordo che altri nostri giuristi in quegli anni di preparazione di nuovi ordinamenti abbiano redatto progetti costituzionali, anche se molti, come è noto, contribuirono come membri dell'Assemblea Costituente, alla elaborazione della nostra Costituzione.

Questi progetti di Trentin rimangono un unicum, anche per la forma di stato e di governo che essi disegnano, e per essere anche ideologicamente fortemente caratterizzati. Basti dire che nell'art. 1, intitolato Principio Generale, corrispondente all'articolo della nostra costituzione in cui si aggiunge alla formula di rito "repubblica democratica" la famigerata connotazione «fondata sul lavoro», si legge che la repubblica «federale» erige a "criterio supremo» per la legittimazione del funzionamento dello stato «i grandi principi della libertà della persona, dell'autonomia istituzionale, della proprietà collettiva e della giustizia sociale». (Questa caratterizzazione ideologicamente così marcata non esiste nel progetto francese che fu scritto prima).

La libertà della persona era un tipico principio delle costituzioni liberali, la proprietà collettiva era allora il principio sancito nell'unica costituzione socialista del tempo, quella sovietica.

Nonostante il federalismo di Trentin fosse volto più al problema dell'organizzazione interna dello stato che a quello dell'organizzazione internazionale, il problema dell'Europa non era stato dimenticato. Sin dal 1930 in Antidemocrazia scriveva: «Se il fascismo è l'anti-Europa, l'Europa non può non essere l'antifascismo». Combattere il fascismo voleva dire combattere il gretto e aggressivo nazionalismo che era stato certamente una delle cause della prima guerra mondiale che avrebbe portato alla seconda.

Nel saggio Libérer et Fédérer sostiene che la rivoluzione in corso non avrà adempiuto il suo compito se non quando l'intera umanità avrà raggiunto la sua unificazione istituzionale in un'unica forma federale (l'ideale che va da Kant fino ai giorni nostri), e gli stati, completando il processo che è all'origine della loro formazione, potranno dissolversi nello stato. Nei due progetti di costituzione il primo articolo prevede che rispettivamente la Francia e l'Italia rivendichino la dignità e il titolo di membri fondatori della repubblica europea. Nel programma del movimento 
Libérer et Fédérer si legge: «Il governo rivoluzionario dovrà preparare, in collaborazione coi governi degli altri paesi liberati dal fascismo e dal nazismo, le basi di una federazione europea fondata sulla libertà, la pace e la prosperità».

Sono ben note le difficoltà di vita attraverso cui Trentin passò dalla rinuncia per libera volontà alla cattedra molti anni prima che il governo fascista imponesse il giuramento all'esilio a quarant'anni, con la famiglia e i figli in Francia dove esercitò per anni il mestiere del tipografo e quello del libraio a Tolosa, che diventò un luogo d'incontro tra antifascisti, specie durante la guerra di Spagna e il regime di Vichy e fece dire a Jean Cassou che era diventato «il centro principale dell'intelligenza antifascista di Tolosa».

In una commemorazione tenuta a Roma nel 1944, Lussu, in uno stupendo ricordo dell'amico, scrisse che la libreria di Trentin era diventata una specie di Società delle Nazioni. Centro della nostra emigrazione che sfuggiva ai tedeschi e punto di riferimento di tutta la fiumana di intellettuali, scrittori, artisti, uomini politici, ebrei già fuggiaschi dal Belgio, dalla Polonia, dalla Germania ecc. casa Trentin era diventata il quartier generale dei capi di tutti i partiti francesi di sinistra. E la moglie, Beppa Nardari, la devota e buona compagna della sua vita, sempre presente.

Come abbia fatto in questi frangenti, non godendo dell'ozio degli intellettuali, né avendo una cattedra universitaria, come accadde in seguito a molti nostri docenti cacciati dall'insegnamento per le leggi razziali, è cosa che suscita la sorpresa e l'ammirazione in chiunque si sia avvicinato, o attraverso i libri o attraverso la conoscenza delle vicende personali, a questa figura eccezionale di uomo di studi e d'azione, che non dissocia mai l'impegno dello studioso da quello del militante, per il quale, riprendendo i titoli dei due famosi saggi di Max Weber, tanto la scuola quanto la politica sono insieme, e non disgiuntamente, una professione e una vocazione. Non disgiuntamente, e però non confuse. Trentin sapeva perfettamente dare alla scienza quel che è della scienza e alla politica quel che è della politica. Non concepiva un sapere che non fosse utile (secondo l'insegnamento di uno dei suoi autori, Carlo Cattaneo) e insieme non concepiva una politica che non fosse illuminata dallo studio della storia, dell'economia, del diritto, della società.

Uno dei più illustri giuristi francesi, che volle scrivere un'ampia prefazione a La Crise du Droit et de l'État, François Gény, riconobbe l'esemplarità di questa rara sintesi di energia intellettuale e di coraggio civile, scrivendo: "Attraverso tutti gli sviluppi della sua opera regnava un sentimento intenso dell'eminente dignità di tutti gli uomini e un soffio generoso di libertà». Nelle conclusioni, dopo aver espresso la sua ammirazione per lo sforzo compiuto dal professor Trentin per mettere in luce 
e penetrare gli enigmi della vita sociale, dichiara: «La mia ammirazione si eleva sino a un'emozione simpatetica, che non può che accrescere la mia riconoscenza, quando penso alle condizioni dolorose e difficili che un esilio, coraggiosamente e mirabilmente accettato, ha imposto alla realizzazione di questo sforzo. La nuova opera del prof. Trentin supera di gran lunga il livello ordinario dei lavori scientifici anche i più approfonditi. Diventa un esempio sublime: esempio d'indipendenza di pensiero, di energia morale indomabile, d'alta virtù civica, di fedeltà, senza compromessi né riserve, al puro ideale del diritto».

Vorrei citare ancora un altro grande studioso francese, che fu suo compagno della Resistenza, Jean-Pierre Vernant, il quale scrisse: «Silvio Trentin incarnò per noi tutti questa fermezza, questa rettitudine di vita, il cui esempio era così necessario per far vivere la speranza nelle ore buie della guerra e dell'occupazione. Coloro che lo hanno ben conosciuto hanno saputo vedere nella forza e nella limpidezza del suo carattere una fine sensibilità, un grande calore di sentimento".

Come sapete il maggior biografo di Trentin è uno studioso americano, Frank Rosengarten, cui dobbiamo la monografia Silvio Trentin dall'interventismo alla Resistenza pubblicata da Feltrinelli nel 1980, il quale afferma: «Se la sinistra italiana dalla fine della seconda guerra mondiale a oggi è riuscita a mantenere vivi gli ideali della democrazia socialista ... è perché uomini come Silvio Trentin durante gli anni dell'opposizione al fascismo hanno gettato le basi per una politica socialista indipendente. Trentin ha dato un esempio che ancora continua a ispirare i socialisti che amano la libertà».

In tutte e tre le citazioni, che ho tratto da uomini di studio e non da politici di partito, ritorna la parola esempio. Trentin come esempio. Perché? La ragione non è tanto difficile da scoprire. Per Trentin la politica era una cosa seria. L'attività politica non era soltanto volontà di dominio ma era prima di tutto volontà di giustizia.

Mi sembra talora che parlare di ideali a proposito dell'azione politica sia fare un discorso fuori del tempo, che viene accolto se non proprio con fastidio con insofferenza o incredulità. Eppure ho la convinzione che senza grandi ideali si fa soltanto della piccola politica, e la piccola politica finisce di divorare se stessa. Vorrei sommessamente dire a coloro ai quali gli ideali sembrano dar fastidio, che cresce, al contrario, il fastidio per coloro che vedono nella lotta politica solo una lotta per il potere.

La democrazia è nata da grandi contese ideali del passato ed è stata ritrovata, dopo che l'avevamo perduta, attraverso non meno difficili e sanguinose battaglie ideali, grazie anche a uomini come Trentin. Un popolo senza ideali può vivere benissimo sotto una dittatura. Anzi, la dittatura è la forma di governo migliore per un popolo di scaltri e di indifferenti.

Una democrazia sopravvive se la gente ci crede. Ma perché ci creda, dobbiamo fare tutti quanti lo sforzo di sollevare più in alto il nostro 
sguardo, oltre gli interessi e gli affari quotidiani. Ci riusciremo più facilmente se ci ricordiamo ogni tanto che un popolo senza grandi ideali morali è un popolo di servi anche se vive in un regime formale di libertà, e non dimenticheremo gli uomini come Silvio Trentin, che al contrario di coloro che sono servi anche in tempi di libertà, seppero essere liberi in tempi di servitù. 



\section{FEDERALISMO E LIBERTÀ ${ }^{\star}$}

1. All'esposizione del proprio pensiero federalistico Silvio Trentin dedicò gli ultimi scritti prima della morte, avvenuta il 12 marzo 1944. Di questi, i principali sono: l'opera prevalentemente storica, Stato Nazione Federalismo, concepita intorno al 1940, scritta in italiano, pubblicata per la prima volta in edizione clandestina presso la casa editrice "La Fiaccola» di Milano, nel 1945, come n. 1 di una "Biblioteca di cultura politica», con prefazione di Mario Dal Pra; il lungo saggio di carattere prevalentemente teorico, Libérer et Fédérer, scritto in francese intorno al 1942, tradotto poco dopo in italiano da Antonio Giuriolo ${ }^{1}$, ma rimasto inedito sino al 1972, quando fu pubblicato nel volume di Scritti inediti, nella collana «Studi e Documenti», promossa dal Centro Studi Piero Gobetti, presso l'editore Guanda di Parma, coi contributi di Emilio Lussu, Hans Werner Tobler, e la bibliografia degli scritti di Trentin a cura di Paolo Gobetti; i due progetti di costituzione federale per la nuova Francia e la nuova Italia, scritti rispettivamente in francese e in italiano, negli stessi anni, e pubblicati anch'essi per la prima volta nel volume testé citato di Scritti inediti.

Il tempo di questi scritti coincide con quello della creazione del movimento della Resistenza francese, Libérer et Fédérer, di cui Trentin fu uno dei fondatori e dei principali animatori, certamente il più agguerrito teorico, e il cui giornale clandestino, avente per titolo anch'esso «Libérer et Fédérer» e per sottotitolo «Organe du mouvement révolutionnaire pour la libération et la reconstruction de la France»², apparve per la prima vol-

* Introduzione a S. Trentin, Federalismo e libertà. Scritti teorici 1935-1943, Marsilio, Venezia 1987, pp. IX-XXXVII.

1 Vedi A. Trentin, Antonio Giuriolo. Un maestro sconosciuto, Vicenza, Neri Pozza, 1984, p. 110 e p. 113. [Si veda: R. Camurri (a cura di), I quaderni di Antonio Giuriolo, Marsilio, Padova 2016].

${ }^{2}$ Dal n. 12 dell'ottobre 1943 il sottotitolo diventa: «Organe du Mouvement révolutionnaire pour la République socialiste et fédéraliste», pur mantenendo il motto iniziale «Gagner la guerre et gagner la paix». Dei pochi numeri apparsi di questo giornale, diventato rarissimo, è stata fatta una riproduzione fotostatica, con presentazione di M. Dreyfus, pubblicata dal Centre d'Études et de Documentation sur l'Émigration italienne (CEDEI), Parigi 1985, con il concorso dei comuni di Venezia e di Jesolo, e del 
ta con la data storica del 14 luglio 1942, e un programma in cui il movimento viene presentato come un movimento rivoluzionario che deve lasciarsi alle spalle definitivamente la politica dei vecchi partiti, ormai morti, ed ispirarsi alle idee espresse sinteticamente in queste perentorie enunciazioni: «Liberare la Francia e l'Europa dall'invasione fascista e nazista e federare i popoli europei per evitare il ritorno di nuove guerre. Liberare la Francia dallo sfruttamento capitalistico e federare gli operai e i contadini, i tecnici e gli intellettuali, i commercianti e gli artigiani affinché organizzino la produzione nell'interesse del popolo. Liberare la nazione dagli antichi partiti che dividono il popolo e dai demagoghi, e federare tutti i francesi nel grande esercito della ricostruzione della Francia. Liberare il popolo dal giogo dello stato accentrato, fatalmente orientato verso il totalitarismo e la dittatura e federare gli uomini e le categorie professionali e intellettuali per coordinare la loro attività pur rispettandone il più possibile l'autonomia».

2. Nei riguardi del grande stato territoriale nato in Europa all'inizio dell'età moderna sulla dissoluzione della società medioevale, il pensiero federalistico, che ebbe la sua prima espressione nella dottrina dei padri fondatori degli Stati Uniti d'America alla fine del Settecento e la sua naturale continuazione nell'ideale degli Stati Uniti d'Europa dalla metà dell'Ottocento sino ad oggi, ha sempre combattuto su due fronti: verso l'esterno nella direzione del superamento dello stato nazionale in una nuova forma di stato di stati tendenzialmente universale, a ogni modo in un primo tempo supra-nazionale, verso l'interno nella direzione della disarticolazione dello stato nazionale unitario in una forma nuova e diversa di stato di stati, in cui per "stati» non s'intendono più gli stati nazionali degradati da enti sovrani a parti di uno stato più grande ma parti o territori (Länder) dello stato grande, elevati al rango di stati-membri. Il primo stato di stati nasce, o dovrebbe nascere, da un processo di unificazione verso l'alto; il secondo stato di stati, invece, nasce, o dovrebbe nascere, da un processo di liberazione verso il basso. Questi due processi si richiamano l'uno con l'altro, perché il grande stato territoriale moderno è nato contemporaneamente da due processi opposti, di unificazione verso il basso, come superamento della frammentazione politica della società feudale, e di liberazione verso l'alto come rottura dell'unità tendenzialmente universale dell'Impero.

Centro di ricerca Silvio Trentin. Sul movimento «Libérer et Fédérer», si vedano il saggio di H.W. Tobler, Il pensiero politico di Silvio Trentin, in S. Trentin, Scritti inediti, a cura del Centro studi Piero Gobetti, Parma, Guanda, 1972, pp. 47-101, che riprende e rielabora il più ampio saggio dello stesso autore, Silvio Trentin und die Widerstandsbewegung «Libérer et Fédérer», München 1967, e i capp. 19 e 20 della monografia di F. Rosengarten, Silvio Trentin. Dall'interventismo alla Resistenza, Feltrinelli, Milano 1980, pp. 175-199. 
Pur richiamandosi l'uno con l'altro, questi due aspetti della dottrina federalistica non sono né logicamente né, quel che più importa, storicamente connessi. Vi sono scrittori federalisti su entrambi i versanti: Cattaneo fu sostenitore a un tempo e con eguale forza di convinzione sia degli Stati Uniti d'Italia contro i dottrinari dell'unità nazionale sia degli Stati Uniti d'Europa contro i fautori dell'impero sovranazionale austriaco. Ma nello stesso Cattaneo prevalse, per lo meno nella battaglia politica realmente combattuta, il primo sul secondo. I due federalismi rappresentano effettivamente due esigenze diverse: l'uno di unificazione di ciò che è disunito, l'altro di disunione di quello che è unito. Questa è la ragione per cui possono non incontrarsi e non compenetrarsi nello stesso autore: Mazzini fu certamente un fautore degli Stati Uniti d'Europa, e da lui in gran parte presero ispirazione le Società democratiche per la pace che fiorirono nella seconda metà del secolo scorso, ma non altrettanto degli Stati Uniti d'Italia. Anche le occasioni storiche da cui nascono i due federalismi non sono necessariamente le stesse: il federalismo esterno nasce prevalentemente da una crisi bellica, da una crisi internazionale, da una reazione a un certo sistema di rapporti fra stati sovrani, di cui la guerra è un elemento costitutivo; il federalismo interno nasce invece prevalentemente in una situazione di crisi interna, da una vicenda di disgregazione di uno stato accentrato o monocentrico, anche in seguito a una guerra, sì, ma a una guerra civile, che libera forze compresse da un potere dispotico. Per usare le due grandi dicotomie kelseniane, imperialismo-pacifismo, la prima, indicante le due forme antitetiche di rapporti fra stati, autocrazia-democrazia, la seconda, indicante le due forme antitetiche di rapporti fra governanti e governati all'interno di uno stesso stato, il federalismo esterno è antimperialista e pacifista, quello interno antiautocratico e democratico. Si tratta di due caratteristiche di per se stesse ben delineate e delimitate, ma non per questo necessariamente convergenti: se è improbabile che uno stato dispotico al suo interno sia pacifista nei rapporti con gli altri stati, è invece possibile che uno stato imperialista verso l'esterno sia democratico (e federale) al proprio interno.

Gli scritti federalistici di Trentin appartengono, come si è detto, agli anni in cui la lotta mortale contro il fascismo è nel pieno corso e chi partecipa appassionatamente a questa lotta è convinto della sua imminente fine e progetta le istituzioni dell'avvenire. Alla caduta del fascismo la situazione sarebbe stata tale da provocare una reazione in entrambe le direzioni del pensiero federalistico e quindi da renderlo attuale nella sua duplice versione. I regimi fascisti, infatti, erano stati insieme dispotici all'interno e imperialistici all'esterno; avevano espresso una forte aggressività verso gli altri stati servendosi di un altrettanto forte concentrazione di potere attraverso il partito unico coatto di massa sul territorio nazionale. 
3. Di fatto, il pensiero federalistico di quegli anni si mosse più nella direzione del federalismo esterno che in quella del federalismo interno. Il Manifesto di Ventotene che, elaborato nell'estate del 1941 da Ernesto Rossi, Eugenio Colorni e Altiero Spinelli, ebbe la sua prima pubblica o semipubblica consacrazione nel 1943, presenta un compiuto progetto di federazione europea; di una eventuale riforma dello stato unitario in senso federalistico non v'è alcuna traccia né nel testo del Manifesto né nella prefazione di Colorni. In uno dei primi numeri di «L'unità europea» a Mario Alberto Rollier che sottolinea la necessità di riconoscere anche l'esigenza di un federalismo infra-nazionale accanto a quello supra-nazionale, Spinelli risponde spostando l'accento ancora una volta sul secondo. Luigi Einaudi che sin dal 1918 aveva messo in guardia i governi dal vagheggiare una futura società delle nazioni se questa fosse rimasta allo stadio della confederazione, come infatti rimase, e non avesse preso come modello lo stato federale costituito dalle tredici colonie inglesi dell'America del Nord, rimase fermamente unitario per quel che riguarda l'assetto interno dello stato italiano dopo il fascismo, anche se non contrario alla istituzione delle regioni che peraltro avrebbero dovuto sostituire le province e a ogni modo distinguersi dagli stati-membri di uno stato federale. Umberto Campagnolo, allora segretario generale del Movimento federalista europeo, scrisse nel febbraio 1945 un saggio intitolato Repubblica federale europea, in cui non solo non si pone il problema dell'assetto interno del nuovo stato che dovrà essere ricostruito dall'Assemblea costituente ma sostiene addirittura che il federalismo interno è da condannare come un falso federalismo perché va a ritroso del progresso storico che muove verso sempre più estese unificazioni di stati piccoli o medi e non verso la disarticolazione degli stati esistenti ${ }^{3}$.

L'originalità del pensiero federalistico di Trentin, o, se vogliamo, la sua caratteristica sta nel muoversi nella direzione del federalismo interno molto più che in quella del federalismo esterno. Non già che non si renda conto del nesso dei due problemi e non si affacci anche sul versante del federalismo supra-nazionale che dovrebbe a lunga scadenza sboccare nella costituzione dello stato universale, come vedremo. Ma il tema dominante dei suoi ultimi scritti è il federalismo infra-nazionale. Il problema che lo assilla - un assillo scaturito dalla sofferta esperienza dello stato totalitario che dall'Italia si è esteso nell'intervallo fra le due guerre a gran parte dell'Europa - è quello di trovare un rimedio al dispotismo che comprime la libertà degli individui e l'autonomia dei gruppi. Il suo

${ }^{3}$ Per maggiori particolari rinvio al mio saggio, Il federalismo nel dibattito politico e culturale della Resistenza, in AA.VV. L'idea dell'unificazione europea dalla prima alla seconda guerra mondiale, a cura di S. Pistone, Torino, Fondazione L. Einaudi, 1975, pp. 221236. [Si veda: U. Campagnolo, Repubblica federale europea, introduzione di L. Cedroni, Rubbettino, Soveria Mannelli 2004]. 
progetto è prima di tutto un progetto libertario, ove il principio federativo e le connesse istituzioni federali vengono poste a difesa della libertà dei singoli e dei gruppi contro la tendenza del potere centrale a soffocarle, e solo secondariamente un principio di unificazione di poteri tradizionalmente antagonistici come sono sempre stati nei loro reciproci rapporti gli enti sovrani che non riconoscono al di sopra di sé alcun ente superiore.

Riprendendo ancora una volta la rappresentazione delle due facce della dottrina federale, quella libertaria e quella pacifista, il pensiero federalistico di Trentin è certamente tanto libertario quanto pacifista, ma l'ispirazione libertaria prevale su quella pacifistica. Il suo interesse di studioso di diritto e di politica è rivolto alla critica dello stato nazionale che si è venuto identificando con un potere sempre più monocentrico e alla progettazione di una costituzione di tipo federale che garantisca il libero movimento degl'individui e dei gruppi all'interno dello stato territoriale più che all'idea della federazione europea, ben più diffusa, e forse anche più ovvia, durante e dopo la seconda guerra mondiale. Non è un caso che gli autori cui egli si riferisce siano da un lato Proudhon e dall'altro i federalisti italiani, Cattaneo e Ferrari (ma più Cattaneo di Ferrari, a giudicare dai testi citati) che sono da annoverare tra i fautori del federalismo libertario o democratico ben più del federalismo pacifista, che, per lo meno in Italia, ebbe per padre spirituale, come si è detto, Mazzini.

4. Più volte si è osservato che a questa maggiore propensione per il federalismo democratico egli era predisposto dalla sua formazione di giurista e in particolare dalla sua competenza specifica di cultore di diritto amministrativo, di una branca del diritto di cui uno dei capitoli principali è quello che tratta delle autonomie locali. Non sarà mai sottolineato abbastanza che uno dei termini chiave che ricorrono nelle sue opere federalistiche è appunto "autonomia», e che nell'opera in cui si fa strada per la prima volta la tesi dello stato federale, come rimedio alla crisi del diritto e dello stato, le espressioni che egli usa per dare un nome nuovo al nuovo ordine, sono «ordine delle autonomie»e «ordine degli ordini» ${ }^{4}$. È bensì vero che a questa connessione fra il concetto di autonomia e l'idea dello stato nuovo come ordine delle autonomie egli giunge soltanto nelle ultime opere. In uno degli articoli più importanti del periodo italiano (il decennio 1916-1926), Autonomia, Autarchia, Decentramento (un titolo tripartito come quello del libro federalistico, citato all'inizio),

\footnotetext{
${ }^{4}$ Si tratta di La Crise du Droit et de l'État, L'Eglantine, Parigi 1935. L 'idea dello stato come ordine delle autonomie è esposta nel cap. IV. L 'espressione «ordine delle autonomie», rilevata da Gény nella prefazione, si trova a p. 171, quella "ordine degli ordini», a p. 156. Su questo libro più a lungo al \$9. [Trad. it., La crisi del diritto e dello stato, a cura di G. Gangemi, Gangemi Ed., Roma 2006; con un saggio del curatore, Silvio Trentin, il diritto naturale e la libertà come autonomia, pp. 7-40].
} 
nato come discorso inaugurale all'Istituto di Ca' Foscari nel novembre 1924 l'ultimo anno del suo insegnamento universitario (si dimetterà il 7 gennaio 1926), egli mostra di essere favorevole, seguendo la communis opinio degli studiosi di diritto pubblico del tempo, allo stato unitario, e quindi attribuisce al solo stato, in quanto titolare unico della sovranità, la prerogativa della autonomia, intesa etimologicamente come potere di dare norme a se stessi, mentre agli enti locali concede unicamente, sulla scorta di Santi Romano, la caratteristica dell'autarchia, limitatamente intesa come capacità di amministrare i propri interessi. Inoltre rigetta esplicitamente le tesi neo-federalistiche del tempo «come una espressione di malcontento che reagisce al malcostume amministrativo, rievocando (senza tener conto delle mutate condizioni storiche) la suggestiva bellezza di alcuni sistemi politici propugnati da venerandi maestri nel calore delle dispute che prepararono l'indipendenza del Regno" ${ }^{5}$. I fautori delle tesi neo-federalistiche erano allora i repubblicani, che nell'Italia unitaria rimasero i più fedeli all'insegnamento politico cattaneano. Trentin si riferiva in modo particolare a Oliviero Zuccarini, direttore della rivista di battaglia repubblicana, "Critica politica», che in un articolo della stessa rivista, Possibilità del regionalismo (25 gennaio 1924), aveva escluso che la riforma regionale potesse compromettere l'unità, avendo distinto l'unità come potere unico, di uno solo su tutti, che è «unità oppressiva, unità fascista se si vuole», dall'unità «di consensi, di volontà e di opere» ${ }^{6}$. La stessa critica muove in quegli anni ai regionalisti Piero Gobetti, se pure esprimendo la sua simpatia, non diversamente del resto da Trentin, per la «bella rivista di O. Zuccarini» ${ }^{7}$.

Rispetto allo stato federale, "che emerge per la prima volta, nitido nei suoi contorni, dalle rivoluzioni che sulla fine del sec. XVIII divamparono nel vecchio e nel nuovo mondo", introducendo la distinzione di scuola fra stati semplici e stati composti (composti ma pur sempre stati e non mere associazioni o confederazioni di stati), sostiene la tesi, non nuova fra $\mathrm{i}$ giuristi europei, del carattere meramente transnazionale di questo tipo di stato, che deriva da un "compromesso fra la volontà prepotente di conquistare con qualunque mezzo l'indipendenza, il proposito di ri-

${ }^{5}$ S. Trentin, Politica e amministrazione. Scritti e discorsi 1916-1926, a cura di M. Guerrato, «Opere scelte di Silvio Trentin», Venezia, Marsilio, 1984, p. 345. Sui primi anni dell'attività di Trentin, si veda l'esauriente monografia di M. Guerrato, Silvio Trentin, un democratico all'opposizione, Vangelista, Milano 1981, e sul discorso citato, in particolare p. 199 e ss. [Per un aggiornamento sulla letteratura si veda Bibliografia di e su Trentin in appendice].

${ }^{6}$ Questa frase è riportata in nota dallo stesso Trentin, Op. cit., p. 346, nota 10; Zuccarini deformerebbe il contenuto della sovranità, che neppure Duguit, pur con le acute osservazioni sui moderni orientamenti di diritto pubblico, è riuscito ad infirmare.

7 P. Gobetti, I repubblicani, in «La rivoluzione liberale», II, n. 10, 17 aprile 1923, ora in Scritti politici, Einaudi, Torino 1960, pp. 491-492. 
spettare [...] le particolari autonomie nazionali, e la necessità infine di attribuire un assetto organico e perciò vitale alle nuove consociazioni politiche» ${ }^{8}$. In base a questa interpretazione dello stato federale critica coloro che ritengono di trovare oggi conforto «nell'opera generosa dei federalisti italiani, dei quali fu apostolo infaticabile ed indimenticato Carlo Cattaneo" per promuovere l'autonomia politica e non soltanto amministrativa delle regioni: "A buon diritto l'idea federale poteva essere bandita come segnacolo di riscossa nel momento in cui le condizioni dell'Italia, asservita e divisa, sembravano contrastare al rapido successo di un programma unitario", ma oggi, «dopo oltre settant'anni di convivenza unitaria», appoggiare le nuove tendenze autonomistiche non potrebbe giustificarsi se non a patto di voler sostituire l'attuale ordinamento con un «nuovo tipo di stato a composizione molteplice», composto di diversi e distinti enti collettivi caratterizzati da autonomia sia di organizzazione sia di poteri ${ }^{9}$. L'assurdità di questa posizione deriverebbe, secondo Trentin, anche dal fatto che il processo di unificazione delle sparse membra di una nazione in un ente collettivo unitario è irreversibile, perché lo stato semplice unitario, attuando il più perfetto equilibrio tra le forze sociali coesistenti sul medesimo territorio, costituisce «la meta finale» verso cui tendono inevitabilmente a gravitare le varie forme di organizzazione politica delle società umane storiche. Sotto questo aspetto lo stato federale sarebbe, non soltanto rispetto alla sua struttura giuridica ma anche storicamente, uno stato di transizione, e di transizione proprio perché risultante da un compromesso destinato a non durare indefinitamente.

5. Orbene, è proprio al «nuovo tipo di stato a composizione molteplice» che Trentin guarda con speranza e fiducia negli ultimi scritti, disegnandone le principali linee di struttura nei due progetti di costituzione per la Francia e l'Italia liberate, e in tal modo rovesciando completamente il punto di vista della prolusione del 1924. Lo riconosce egli stesso molto francamente in una pagina autobiografica di Stato Nazione Federalismo, là dove scrive: «Confesso che, vittima di una deformazione professionale assai diffusa fra i giuristi e troppo penetrato ancora dei pregiudizi di un insegnamento eccessivamente rispettoso delle forme pure del diritto, anch'io per un momento ho creduto all'esistenza e all'autorità di una siffatta legge regolativa dell'evoluzione degli istituti giuridicopolitici», secondo cui «il tipo di stato semplice-unitario attua il più perfetto equilibrio [...] fra le forze sociali coesistenti sul medesimo territorio e costituisce perciò la meta finale verso cui è giocoforza debbano a poco a poco

${ }^{8}$ Op. cit., p. 340.
${ }_{9}$ Op. cit., p. 341. 
gravitare [...] le varie particolari forme di organizzazione adottate nella pratica dalle diverse società politiche» ${ }^{10}$.

Il superamento del "pregiudizio» formalistico, proprio dei giuristi della sua generazione, non era stato certamente l'unica ragione della conversione, se si può chiamare così il mutamento radicale di opinione su un tema centrale come quello della miglior forma di stato. Ne era stato tutt'al più un presupposto. Anche Trentin si era formato, come i suoi maestri e i suoi compagni di studio, alla scuola del diritto pubblico tede$\mathrm{sco}^{11}$, che era stata improntata al più rigoroso tecnicismo e aveva trovato la sua massima espressione nell'opera di Kelsen, che egli mostra di conoscere molto bene. In Francia il contatto personale con alcuni dei maggiori giuristi francesi del tempo (Julien Bonnecase scrive la prefazione a Les transformations récentes du droit public italien, 1929, e François Gény a La Crise du Droit et de l'État, 1934), uno studio più approfondito dei loro scritti (due fra i maggiori interlocutori della sua opera teorica principale sono Maurice Hauriou e Léon Duguit), il grande interesse, se pure criticamente orientato, per la teoria del «droit social» di Georges Gurvitch, avevano certamente contribuito ad allargare i suoi orizzonti di giurista.

Però la ragione fondamentale del mutamento di prospettiva deve essere ricercata nel carattere stesso della storia europea dell'«ère des tyrannies». Lo scoppio della seconda guerra mondiale aveva ormai rivelato allo studioso della crisi dello stato e del diritto che la naturale tendenza dell'organizzazione statale verso l'unificazione dei centri di potere aveva finito per generare il mostro dello stato totalitario, non solo in Italia e in Germania, nei paesi fascisti, ma anche nel grande stato della prima rivoluzione socialista, e gli aveva fatto capire, nella mancanza di strumenti adatti di analisi e di ricerca, soprattutto attraverso la energica lezione delle cose, ciò che subito dopo la fine della prima guerra mondiale e nei

10 S. Trentin, Federalismo e libertà, cit., pp. 143-144.

11 Trentin aveva trascorso quasi un anno in Germania, fra il settembre 1913 e il luglio 1914, per perfezionarsi alla scuola del prof. Fritz Fleiner, d'origine svizzera, che insegnava diritto amministrativo a Heidelberg ed era un ammiratore della struttura federale dell'Impero tedesco e, come svizzero, della struttura federalistica dello stato in generale. Così Rosengarten, Silvio Trentin, cit., p. 47. Al prof. Fleiner Trentin dedica l'opera principale dell'esilio, La crise du droit et de l'État, e nell'Avant-Propos scrive: «Je dédie ce livre au professeur Fritz Fleiner, Recteur de l'Université de Zurich, Président de l'Institut international de droit public. À la veille de la Grande Guerre, j'ai eu le bonheur d'approcher, dans la vieille cité universitaire de Heidelberg, ce maître éminent et de puiser sans compter à son enseignement lumineux. Dans les heures sombres de la proscription, j’ai eu le bonheur, encore plus grand, de jouir du réconfort ineffable de sa sympathie agissante». Suo maestro italiano era stato, all'università di Pisa, Giovanni Vacchelli, che, a giudizio di Feliciano Benvenuti, non aveva seguito ciecamente il metodo dommatico, come risulterebbe dalla sua prima opera, Le basi psicologiche del diritto pubblico. Cito dal discorso pronunciato dal rettore dell'Università di Ca' Foscari, in occasione dell'intitolazione dell'Aula Magna a Silvio Trentin il 5 aprile 1976. 
primi anni dell'avvento del fascismo pochi avevano previsto: l'instaurazione di regimi autocratici sulle rovine dei più fragili regimi democratici era non già un fenomeno effimero ma il prodotto di una crisi storica.

Trentin non sconfessava affatto l'argomento principale in base al quale aveva allora rifiutato il federalismo, l'argomento tratto dalla storia, secondo cui non si era mai dato il caso di uno stato unitario che si fosse tramutato in stato federale, mentre era certa la tendenza di ogni stato inizialmente composto a trasformarsi in uno stato sempre più unificato e unitario. Una delle tesi centrali di Stato Nazione Federalismo è che il processo di unificazione del potere era stato continuo, e si era manifestato nel corso del sec. XIX con una costanza estremamente significativa. Ma si trattava di una tendenza evolutiva o involutiva? Ecco il punto. Ciò che cambiava allo scoppio della seconda guerra mondiale, per effetto della preponderanza degli stati non democratici, non era tanto il giudizio di fatto, che restava confermato oltre misura dalla comparsa, dall'espansione e dalla persistenza degli stati totalitari, ma era il giudizio di valore. Certo, la tendenza verso l'unificazione era stata costante. Ma era una tendenza che si era dimostrata alla prova dei fatti nefasta. Era una tendenza che doveva essere capovolta. Così il problema veniva spostato dall'essere al dover essere, e da storico diventava eminentemente politico.

6. Il rapporto fra l'ispirazione centrale del libro del 1940 e la situazione storica è evidente. Ma occorre aggiungere subito che Trentin non aveva aspettato lo scoppio della guerra per rendersi conto che, se la tendenza secolare era stata nella direzione di un sempre più marcato monocentrismo, era ormai necessario un risoluto capovolgimento di rotta di fronte alle conseguenze perverse dello stato monocentrico, sia rispetto alle libertà degl'individui (il dispotismo) sia rispetto al turbamento della pace internazionale (l'imperialismo).

Il momento di svolta rispetto alle idee precedenti, di orientamento democratico radicale, è rappresentato dal libro, scritto in italiano, pubblicato a Marsiglia nel 1933 (l'anno, non si dimentichi, dell'avvento di Hitler al potere), Riflessioni sulla crisi e la rivoluzione. Si trattò, com'è stato detto esattamente, di una duplice svolta, sia rispetto al metodo sia rispetto al contenuto ${ }^{12}$ : rispetto al metodo, nel passaggio dal riformismo, cui egli è ormai incline ad attribuire tutti gl'insuccessi dei partiti socialisti tradizionali (si veda ad esempio il saggio Bisogna decidersi del 1934 ${ }^{13}$ ), al riconoscimento della necessità di una rivoluzione che si proponga d'instaurare nuove istituzioni economiche e politiche sulle rovine del capi-

12 G. Paladini, Silvio Trentin, in «Belfagor», XXXII, 1977, n. 4, pp. 409-428.

${ }_{13}$ Pubblicato in "Quaderni di Giustizia e Libertà", n. 11, giugno 1934, pp. 99-108, ora in S. Trentin, Antifascismo e rivoluzione. Scritti e discorsi (1927-1944), a cura di G. Paladini, «Opere scelte di Silvio Trentin», Marsilio, Venezia 1985, pp. 275-286. 
talismo; rispetto al contenuto, nel passaggio dalla democrazia radicale al socialismo, e di conseguenza all'accettazione incondizionata dell'economia collettivistica, della politica di piano, e alla presentazione dell'Unione Sovietica, se pure con sostanziali riserve, che non verranno mai meno, per la mancanza di garanzie dei diritti di libertà, come un indispensabile alleato per la futura rivoluzione italiana.

Che si fosse trattato di una svolta, di una vera e propria "crisi ideologica ${ }^{14}$, egli stesso riconobbe con la sua solita sincerità verso se stesso e con la ben nota lealtà verso gli altri, e con la volontà, talora anche spietata, di autocritica, nell'articolo con cui rispose alla recensione che del libro aveva scritto Emilio Lussu, il «fraterno amico», sul n. 7 dei "Quaderni di Giustizia e Libertà»" : "Confesso - egli scrive - che sono soprattutto le esperienze originali maturatesi nel corso della mia proletarizzazione forzata che mi hanno sospinto a rivedere e a controllare con sempre più larga spregiudicatezza le posizioni da me occupate e gli obiettivi da me perseguiti nella battaglia nella quale siamo tutti impegnati». Con la frase "proletarizzazione forzata», allude agli anni passati ad Auch esercitando il mestiere del tipografo presso la tipografia di Th. Bouquet, anni peraltro ricchissimi di fecondo lavoro intellettuale. Poco più oltre dichiara che il suo proposito nello scrivere le riflessioni sulla crisi era stato unicamente quello di «far parte ai miei compagni di lotta di un mio particolare stato di spirito, in qualche guisa interessante, date le mie particolari origini e i miei precedenti, nella speranza che essi fossero incitati a ripetere la mia stessa esperienza» ${ }^{16}$.

I due concetti di crisi e di rivoluzione apparivano ormai a Trentin strettamente congiunti, inseparabili: la crisi era tanto grave che non se ne poteva uscire se non con un processo rivoluzionario. Aveva però ragione Lussu nell'osservare che ancora prima che in queste «riflessioni» l'amico aveva lasciato intravvedere il nuovo corso delle sue idee nel saggio La libertà e le sue guarentigie del 1932, in cui, ancora una volta autocriticamente, aveva denunciato l'illusione che egli stesso aveva per anni coltivato che si potesse riorganizzare su basi autonome la vita locale italiana «per riforme graduali, evitando con cura transizioni troppo brusche»" ${ }^{17}$. Quale

${ }^{14}$ Così Rosengarten, Silvio Trentin, cit., p. 109.

${ }^{15}$ Questa recensione è stata ripubblicata nel volume: E. Lussu, Lettere a Carlo Rosselli e altri scritti di Giustizia e Libertà, a cura di M. Brigaglia, Libreria editrice Dessi, Sassari 1979, pp. 177-181.

16 Sugli obiettivi della rivoluzione italiana, in "Problemi della rivoluzione italiana», nn. 21-22, Marsiglia, marzo 1934, ora in Antifascismo e rivoluzione, cit., pp. 251-273. I brani citati sono rispettivamente a p. 252 e p. 253.

${ }_{17}$ Questo saggio è ora compreso nel volume Antifascismo e rivoluzione, cit., pp. 67-94. Vi si legge infatti: «È mio avviso che la futura repubblica italiana sarà vitale solo in quanto saprà moltiplicare, avendo riguardo alle esperienze storiche nazionali, e salvaguardare, con previdente saggezza, i centri attivi e operanti della sua vita politica» (p. 88). Segue 
era la principale ragione della caduta dell'illusione? La risposta non era più dubbia: la instaurazione di un regime come quello fascista che aveva distrutto la vecchia impalcatura dimostratasi troppo fragile per resistere alla tempesta che l'aveva investita. Di qua la conclusione che non poteva essere espressa in forma più perentoria: «La ricostruzione dovrà dunque farsi dal nulla, ab imis. Guai a noi se nella fretta di riedificare eleveremo la repubblica sulle fondamenta del vecchio stato per sempre scomparso ${ }^{18}$.

7. Per rendersi conto dunque pienamente dello «stato di spirito» con cui Trentin aveva abbandonato le vecchie idee, e stava elaborando le nuove, occorre tener presenti non solo l'effetto di un'esperienza di vita che gli aveva consentito di conoscere direttamente la condizione operaia ma anche la situazione storica oggettiva che aveva trasformato profondamente la società europea.

Di questa situazione, il cui maggiore fattore di mutamento era stato il fascismo, egli si occupò da giurista, da storico, e, ben s'intende, con un impegno politico diretto, nei primi anni dell'esilio sino al 1933, durante i quali s'impose il compito di far conoscere il nuovo regime ai francesi e, attraverso la Francia, ai «buoni europei». Per cinque anni consecutivi pubblica, ogni anno, opere di critica politica, costituzionale, economica del fascismo: L'aventure italienne nel 1928, Les transformations récentes du droit public italien nel $1929^{19}$, Antidémocratie ${ }^{20}$ nel 1930, Aux sources du fascisme del 1931, Le fascisme à Genève nel 1932, circa duemila pagine che ancor oggi costituiscono un documento, una fonte e una testimonianza di enorme interesse per gli storici che le hanno ingiustamente, se non dimenticate, poco utilizzate ${ }^{21}$.

Dal primo libro in cui, come appare chiaramente dallo stesso titolo, il fascismo è interpretato come un evento eccezionale nella storia dell'I-

la pagina di autocritica: «Sino a qualche anno fa io credevo che il mezzo più sicuro per organizzare rapidamente su basi autonome la vita locale italiana, consistesse nel procedere per riforme graduali evitando con cura transizioni troppo brusche. Varie ragioni suffragavano allora questa mia opinione: l'inesistenza pressoché dovunque di una chiara coscienza regionale, i sopravviventi vivaci antagonismi provinciali, il disquilibrio economico-sociale fra le varie zone della penisola, l'attitudine di alcune istituzioni esistenti a fornire utili punti d'appoggio al nuovo ordinamento. Oggi questi argomenti diversi sono caduti o, nella migliore delle ipotesi, hanno perduto gran parte del loro valore. Il fascismo ha creato dappertutto il deserto e la morte» (pp. 88-89).

${ }^{18}$ Op. cit., p. 89.

${ }_{19}$ Quest'opera è apparsa in traduzione italiana col titolo Dallo Statuto albertino al regime fascista, a cura di A. Pizzorusso, come primo volume delle "Opere scelte di Silvio Trentin", Marsilio, Venezia 1983.

${ }^{20}$ Di quest'opera sono stati tradotti e riprodotti due capitoli in Antifascismo e rivoluzione, cit., pp. 21-53.

${ }^{21}$ Si veda ad esempio il noto libro di R. De Felice, Le interpretazioni del fascismo, Laterza, Bari 1969, in cui è preso in considerazione il primo dei cinque libri citati. 
talia, e quindi destinato a esaurirsi rapidamente, forse senza lasciare profonde tracce nel tessuto costituzionale dell'Italia liberale, le cui istituzioni nel secondo libro vengono difese contro le aberrazioni costituzionali del nuovo regime, la critica di Trentin si era fatta sempre più serrata via via che il regime si veniva consolidando, e scopriva, da un lato, che esso rappresentava sempre più non tanto un' "avventura italiana» ma una profonda crisi della società europea e, dall'altro, che le tanto vantate istituzioni liberali si erano dimostrate molto più fragili di quel che i suoi difensori ad oltranza, ed egli medesimo, avevano in un primo tempo creduto. Nella terza opera, sopra citata, è costretto a constatare che l'idea democratica si va estenuando dappertutto e che la esperienza italiana, che sembrava dovesse avere la durata di un lampo, «avvinghiata intimamente alle drammatiche vicissitudini della crisi che colpisce un'intera civiltà» che sta generando una nuova età, è diventata una grandiosa prova di laboratorio i cui risultati avrebbero esercitato un'influenza decisiva sulla scelta della futura organizzazione sociale ${ }^{22}$; nella quarta il nesso già intravisto ma ormai evidentissimo fra l'alta borghesia e il fascismo, la interpretazione del fascismo come baluardo della plutocrazia, rivela ormai chiaramente un atteggiamento di fronte al regime che non è più soltanto di critica politica (il fascismo come dittatura) ma anche di critica sociale (il fascismo come difesa di classe) e di conseguenza un'opposizione che, mirando a una profonda trasformazione della società, fa prevedere che l'uscita della crisi non potrà avvenire se non attraverso un processo rivoluzionario. L'ultima opera dedicata all'analisi della politica estera tende a dimostrare che lo sforzo del governo fascista è stato quello di gettare nel disordine le relazioni internazionali allo scopo di far fallire ogni piano di stabilizzazione, svolgendo un'attività essenzialmente antisocietaria non ostacolata dalla colpevole debolezza delle nazioni democratiche.

In tutte queste opere di analisi e di critica del fascismo, il problema di fondo è la contrapposizione democrazia-dittatura, la difesa ad oltranza della democrazia contro il morbo dell'antidemocrazia che minaccia le libere istituzioni in Europa. Sono opere di critica e non ancora di proposta. Ma è attraverso questa critica sempre più radicale, sempre più preoccupata dell'avvenire della libertà, sempre più consapevole della vastità e della profondità del male, che nascono le riflessioni sulla crisi e sulla rivoluzione.

8. Che l'ordine nuovo, implicante il passaggio dal sistema capitalistico a un sistema di economia collettivistica, secondo la formula «le terre ai contadini, le fabbriche agli operai e il commercio allo stato", non possa avvenire se non per via rivoluzionaria è il tema centrale, appunto, del-

${ }^{22}$ Da Antifascismo e rivoluzione, cit., p. 38. 
le Riflessioni sulla crisi e la rivoluzione. In quest'opera già insegue l'ideale, che lo ispirerà ancora più nettamente negli ultimi scritti, di una terza via fra la soluzione plutocratica, identificata storicamente negli Stati Uniti, e quella comunista, identificata nell'Unione Sovietica: soluzioni che per diverse ragioni finiscono per negare lo stesso principio dell'autonomia che si erano proposte di salvaguardare.

Autonomia è il concetto-chiave dell'opera. Trentin non parla ancora esplicitamente di federalismo, ma il termine appare nella replica a Lus$\mathrm{su}$ (che, conviene accennarlo in questa sede, si considerava egli stesso un federalista) ${ }^{23}$, in cui riprende il tema del libro per chiarire meglio il suo pensiero e sgombrare la polemica da alcuni equivoci: «L'organizzazione dello stato moderno non può essere caratterizzata dal sindacalismo, ma dal federalismo, cioè a dire dall'autonomia di tutti i centri unitari di vita collettiva esistenti all'interno del gruppo storico che esso coordina, siano o non siano essi a base sindacale» ${ }^{24}$. Federalismo e stato delle autonomie sono evidentemente ormai concetti scambiabili. Lo stato delle autonomie, così come viene presentato nelle Riflessioni, è un preannuncio dei futuri progetti di costituzioni federali. Il nuovo ordinamento che dovrà nascere da un violento processo rivoluzionario rappresenterà il trionfo integrale del principio di autonomia. Sarà questo principio di autonomia che distinguerà la futura costituzione della repubblica italiana (come si vede, l'idea di elaborare una nuova costituzione ha profonde radici) da tutte le costituzioni precedenti, in quanto esso possa applicarsi ai diversi livelli in cui si esplica ogni attività dell'individuo e ogni attività dei gruppi di cui l'individuo viene a far parte: «Autonomia del cittadino; autonomia dell'imprenditore; autonomia dell'azienda; autonomia del sindacato; autonomia delle collettività territoriali, siano esse piccole o grandi». A chiusura di questi cerchi concentrici, autonomia dello stato, la quale «non può sussistere se non in funzione dell'autonomia dei singoli elementi di quel mondo composito del quale esso effettua l'unificazione e in confronto del quale la sua volontà diventa legge» ${ }^{25}$. Mancano i particolari istituzionali che egli preciserà in seguito nei progetti di costituzione, ma il disegno generale di uno stato come è lo stato federale, composto di autonomie progressivamente ascendenti dagli enti collettivi minori, sia territoriali sia professionali, verso l'ente maggiore che tutti li regola non comprimendoli, è ormai chiaramente delineato, e in tal modo il passaggio dalla prima fase alla seconda fase del suo pensiero è avvenuta. Mentre nell'articolo del 1924, come si è visto, l'attributo dell'autonomia

${ }^{23}$ Sul federalismo di Lussu si veda il bel libro di G. Fiori, Il cavaliere dei Rossomori. Vita di Emilio Lussu, Einaudi, Torino 1985, specie l'ultimo capitolo, pp. 366-375.

${ }^{24}$ Sugli obiettivi della rivoluzione, cit., p. 264.

${ }^{25}$ In Antifascismo e rivoluzione, cit., p. 212 (nell'edizione originale il brano si trova a p. 152). 
era riservato soltanto allo stato, e gli enti minori erano considerati provvisti soltanto di autarchia, ora viene attribuito a tutti i soggetti, individui e gruppi, che compongono il tessuto complicato, intricato, molteplice, di rapporti sociali sottostanti allo stato. Una concezione in cui soltanto lo stato ha l'attributo dell'autonomia è monistica; una concezione in cui l'autonomia è diffusa in tutta la società è pluralistica.

Il tema del pluralismo era in quegli anni all'ordine del giorno del dibattito giuridico e politico in Europa. Con il saggio, L'ordinamento giuridico, di Santi Romano del 1917 (autore che Trentin peraltro critica in più luoghi) era diventato tema di dibattito corrente la teoria della pluralità degli ordinamenti giuridici. In Francia con Hauriou (cui si era ricollegato lo stesso Romano) e con Renard trionfava la teoria della istituzione che era una versione aggiornata, con radici nel pensiero sociale cattolico, del pluralismo sociale e giuridico. Nel 1932 erano apparse a Parigi di Georges Gurvitch la monumentale opera, L'idée du droit social, e l'opera minore di informazione bibliografica, Le temps présent et l'idée du droit social, nelle quali aveva tanta parte il pensiero federalistico di Proudhon, che sarà una delle fonti di Trentin federalista ${ }^{26}$.

9. Il tema del pluralismo, in particolare l'opera di Gurvitch, sono al centro dell'opera teorica maggiore, che è anche quella in cui il suo pensiero federalistico ne esce, nella conclusione, tutto spiegato: La Crise du Droit et de l'État, apparsa nel 1935, quando, licenziato dalla ditta Bouquet nel maggio 1934, si era stabilito con la famiglia a Tolosa, proprietario e gestore della Librairie du Languedoc.

Il libro viene presentato da un'ampia prefazione di François Gény, la cui opera in quattro volumi Science et technique en droit privé positif, uscita tra il 1914 e il 1924, rappresentava il punto più alto cui era giunta la teoria generale del diritto in Francia. Gény inquadrava giustamente l'opera di Trentin che giudicava «nuova e importante» nella crisi delle istituzioni seguita alla grande guerra. Pur criticandone la parte costruttiva come dottrinalmente troppo astratta e praticamente inattuabile, ne metteva in luce la forte ispirazione etica e considerava "particolarmente nuovo e pieno di conseguenze feconde» il capitolo sesto sullo stato, inteso come "ordine delle autonomie», concludendo che "questa forte dottrina viene ad arricchire singolarmente [...] la nostra teoria del diritto e dello stato». Quanto all'autore, provato dall'esilio, egli lo additava ad esempio «sublime» d'indipendenza di giudizio, d'energia morale «indomptable», di «fedeltà senza compromessi né riserve al puro ideale del diritto».

${ }^{26}$ Sull'importanza di questo dibattito mi permetto di recare qui una testimonianza personale: uno dei miei primi lavori è intitolato Istituzione e diritto sociale, e tratta del pensiero di Hauriou, Renard e Gurvitch (in "Rivista internazionale di filosofia del diritto", XVI, 1936, pp. 385-418). 
La definizione dello stato come "ordine delle autonomie", sottolineata da Gény, può ben essere considerata come la continuazione di un motivo dominante in tutta l'opera di Trentin sin dai suoi primi studi di diritto amministrativo e insieme come sbocco di questo tema dominante nella proposta di una nuova forma di stato qui per la prima volta abbozzata. Nel libro la parte storica e critica è di gran lunga prevalente su quella progettuale, esposta soprattutto nell'ultimo capitolo (che è stato riprodotto, in traduzione italiana, nel volume Federalismo e liber$\left.t a^{27}\right)$. L'autore intrattiene un dialogo serrato coi principali giuristi e filosofi del diritto del tempo, Giorgio Del Vecchio e Santi Romano, Léon Duguit e Maurice Hauriou, Rudolf Stammler e Hans Kelsen. Uno dei suoi interlocutori privilegiati è Gurvitch, tanto che l'opera nel suo insieme può entrare a pieno diritto nella storia della fortuna del «diritto sociale» in Francia negli anni trenta. L'ispirazione filosofica generale è sicuramente giusnaturalistica; così si spiega la consonanza con le idee di Del Vecchio e la critica di Santi Romano considerato come uno dei più intransigenti fautori del positivismo giuridico. Anche se Trentin non si sia mai professato marxista, e spesso abbia mostrato la sua insofferenza verso il marxismo ufficiale, ortodosso, chiuso in se stesso, esclusivista, dei comunisti e della dottrina di stato sovietica, cita con onore, a proposito dell'analisi del capitalismo imperialistico, Lenin e Rosa Luxemburg. Filosoficamente Trentin è un assertore della necessità del diritto e dello stato, contro tutti coloro che, sconvolti dalla crisi del diritto e dello stato capitalistici, sono giunti all'aberrante conclusione che si possa fare a meno dell'uno e dell'altro. La sua tesi filosofica centrale è tutta concentrata in questa affermazione: «Lo stato non può organizzarsi se non organizzando la società, e la società, a sua volta, non può essere organizzata secondo il diritto che conformando la sua organizzazione all'imperativo che pone il valore supremo nell'autonomia della persona e in quella degli ordini richiesta dall'esercizio dei suoi attributi ${ }^{28}$. L'unica forma di organizzazione che consente la continua adeguazione fra l'ordine della società e l'ordine dello stato è la democrazia. L'autore che gli offre la più precisa definizione di democrazia è Kelsen il quale, com'è noto, definisce la democrazia in contrapposizione all'autocrazia come la forma di governo caratterizzata dall'autonomia, ovvero dalla tendenziale corrispondenza tra il soggetto che pone la legge e il soggetto cui la legge è destinata, in contrapposto ad eteronomia. Ma a differenza di Kelsen, che della democrazia ha una concezione puramente formale o procedurale, e quindi sostiene che il regime democratico sia compatibile tanto con un sistema di economia capitalistica quanto con

27 Op. cit., p. 332.

${ }^{28}$ La Crise du Droit et de l'État, cit., p. 188. 
un sistema di economia collettivistica, Trentin è giunto ormai alla convinzione che capitalismo e democrazia sono incompatibili e che pertanto occorrerà trovare la soluzione dell'attuale «crisi del diritto e dello stato" in un sistema che riesca a conciliare il collettivismo economico con la libertà politica. Il punto di partenza è pur sempre la constatazione della "pluralisation des ordres juridiques» che caratterizza la società contemporanea e della tendenza al loro inserimento in sistemi sempre più vasti sì da trasformare lo stato del futuro sempre più in un "ordine d'integrazione nel diritto delle forze sociali», ovvero in un "ordine delle autonomie", considerata l'autonomia sia della persona sia dei gruppi come «il valore supremo che il diritto mira a servire» ${ }^{29}$.

La rigenerazione della società, l'uscita dalla crisi, non può avvenire che attraverso il federalismo inteso come «modo di realizzazione, sul piano collettivo, del principio dell'autonomia $"^{30}$, che implica sia una riforma integrale del sistema politico interno sia l'integrazione organica dello stato particolare in un ordine universale. Ancora una volta il principio ispiratore è l'autonomia: "L'autonomia deve essere istituita alla base di ogni attività, all'origine di ogni facoltà e di ogni potere. Sarà in diritto, come essa è di fatto, il fermento vivificatore che solo saprà rendere operanti gli interessi sia degli individui sia dei gruppi ${ }^{31}$.

10. Allo sviluppo di questa idea qui soltanto enunciata in modo perentorio e apodittico, che provoca il giudizio di astrattezza dato da Gény, Trentin dedica, come ho detto all'inizio, le sue ultime opere, precedute dal saggio L'abdicazione della Francia o la fine di un mondo. Note di un sopravvissuto, scritto subito dopo il crollo dell'esercito francese nell'estate del 1940 (la prefazione reca la data del $1^{\circ}$ ottobre 1940), nel quale, dopo aver analizzato le ragioni della sconfitta, termina con un messaggio di speranza, dettato ancora una volta dall'«esigenza incoercibile dell'autonomia, in quanto reagente dissolutore della vecchia compagine statale e fermento generatore della nuova disciplina della vita collettiva» e ispirato alla convinzione che la Francia sia avviata alla «rivoluzione rigeneratrice», intravista da Proudhon nelle sue incursioni nel regno dell'utopia ${ }^{32}$.

Il libro Stato Nazione Federalismo ${ }^{33}$ consiste per la massima parte in una storia dello stato moderno, raccontata attraverso le vicende della mo-

29 Op. cit., p. 332.

${ }^{30}$ Op. cit., p. 476; in Federalismo e libertà, cit., a p. 29.

31 Op. cit., p. 477; in Federalismo e libertà, cit., alle pp. 29-30.

${ }^{32}$ In Scritti inediti, cit., p. 185.

33 Che sia stato scritto nei primi mesi della guerra può essere documentato dal fatto che all'inizio del cap. XIII, dove l'autore delinea i tratti del futuro stato europeo scrive: «Da oltre sei mesi l'ascia è stata dissotterrata e gli eserciti sono costretti quotidianamente a farsi fronte, per saggiare con le armi, in un duello per la vita e per la morte, la solidità 
narchia francese, della rivoluzione francese, della formazione degli stati nazionali durante il sec. XIX, con particolare riguardo al processo di unificazione della nazione italiana, e in parte in una storia delle principali dottrine che ne accompagnano la crescita e ne giustificano la natura di ente sovrano, cioè dotato di un potere sommo che non riconosce al di sopra di sé nessun altro potere. In questa storia delle idee occupa un posto centrale Hegel che era stato elevato in Italia, a torto o a ragione, per influenza principalmente di Gentile, a incontrastato teorico dello stato fascista. Tutta la narrazione è guidata, sin dalle prime pagine, dal presupposto che nel successo strepitoso dello stato monocentrico sia da ricercare «l'origine più diretta, per quanto cronologicamente tanto remota, delle tremende e forse mortali difficoltà nelle quali oggi la civiltà europea sta confusamente dibattendosiı ${ }^{34}$, ed è sorretta dalla convinzione, vero e proprio motivo dominante dell'intera opera di Trentin, che «la società è, per definizione, molteplicità: molteplicità di centri vitali, di sforzi, di realizzazioni, cui corrisponde il sempre mobile e necessario suo frazionamento in gruppi, enti, istituzioni $\aleph^{35}$, e di conseguenza occorre liberare la «reale costituzione pluralistica della società» dall'arbitraria sovrapposizione dello stato concepito non come mero strumento tecnico per garantire «la coesistenza delle autonomie» qual esso è o dovrebbe essere, ma come astratta entità unitaria ipostatizzata, con una sua capacità di volere al di sopra del diritto. Anche se l'opposizione allo stato etico è condotta soprattutto attraverso la critica della filosofia hegeliana, la riduzione qui proposta dello stato a puro strumento tecnico è la più completa inversione della concezione etica dello stato.

Le fonti del pensiero federalistico di Trentin appaiono per la prima volta chiaramente in questo libro. Esse sono da un lato Proudhon e dall'altro i federalisti italiani del Risorgimento, in primis Cattaneo, attraverso il quale «la lotta dal federalismo ingaggiata nel secolo scorso in Italia e in Europa trovò la sua estrema e più drammatica espressione»" ${ }^{36}$. Drammatica, perché il movimento federalista fu sconfitto e trionfò in sua vece lo stato unitario, al di sotto del quale l'Italia peraltro ha continuato a essere comunale o federalista, se pure in una condizione di continua soggezione coatta allo stato monocentrico ${ }^{37}$.

dei titoli contradditori per cui i governi antagonisti rivendicano, ciascuno per proprio conto, le qualità che solo abilitano all'esercizio del primato e il privilegio pertanto di dettar legge al continente» (in Federalismo e libertà, cit., p. 193).

34 Federalismo e libertà, cit., p. 41.

35 Federalismo e libertà, cit., p. 68.

${ }^{36}$ Federalismo e libertà, cit., p. 134.

37 Significativo a questo punto un riferimento preciso all'azione federalistica di Lussu in Sardegna, che considera la sola azione radicale e organica in senso federalistico (Federalismo e libertà, cit., p. 142). 
11. Per comprendere il pensiero federalistico di Trentin lo scritto più importante è indubbiamente il saggio teorico Liberare e Federare il quale, nei primi nove paragrafi, contiene una critica serrata del capitalismo. Questa critica non è di per sé nuova, perché risale almeno alle Riflessioni del 1934 e alla critica del fascismo interpretato come lo sbocco inevitabile dell'economia capitalistica che in un periodo di crisi ha bisogno per risollevarsi di uno stato forte. Nuova, però, in parte, in quanto per la prima volta la stessa critica è condotta con strumenti di analisi economica di cui Trentin si vale ora in maniera più consapevole che negli scritti precedenti. I primi paragrafi infatti sono dedicati alla critica delle varie strade di politica economica che gli stati capitalistici hanno percorso per uscire dalla crisi della fine degli anni venti che li aveva attanagliati.

Nell'ultima parte il saggio presenta con particolari prima d'ora non rilevati le linee fondamentali di una compiuta teoria federalistica dello stato. Questa teoria si propone la difficile conciliazione del collettivismo economico, che dovrà liberare l'individuo come produttore dalla schiavitù del capitalismo ma mette in pericolo la sua indipendenza di cittadino, come l'esempio dell'Unione Sovietica ha ormai rivelato al di là di ogni ragionevole dubbio, con la pratica della libertà. Il federalismo accetta lo stato contro la negazione degli anarchici ma lo trasforma da ordinamento totalizzante in un «ordine degli ordini»: «Lo stato di domani, se vuole realizzare un progresso rispetto allo stato di oggi, sul piano dell'affrancamento dell'individuo e della salvaguardia della dignità della persona, non può essere che federalista nel senso proudhoniano della parola $»^{38}$.

Non è possibile, né sarebbe neppure utile per il lettore, dar conto preciso in questa sede della complessa struttura di questo stato che vuole essere insieme massimamente collettivista in economia e massimamente democratico in politica, quale viene descritta per sommi capi nel saggio e poi articolata minutamente nei progetti di costituzione sulla base dell'idea direttiva secondo cui «la logica del federalismo esige che il principio ch'esso pone a regola e misura necessarie delle relazioni della vita sociale, sia applicato fino a esaurire tutti i problemi che si riferiscono alle manifestazioni di qualsiasi forma di convivenza» ${ }^{39}$. Basti ricordare che il principio dell'autonomia delle singole parti di cui si compone lo stato riguarda non soltanto gli enti territoriali come i comuni, le province, le regioni, ma anche le singole branche di attività economica, le imprese agricole, le fabbriche, le agenzie di credito, gli enti culturali e così via, e che lo stato come ordine delle autonomie o ordine degli ordini deve rendere possibile la coesistenza di tutti questi enti attraverso una struttura istituzionale a piramide nella quale si passa dagli enti primari a quelli

${ }^{38}$ Federalismo e libertà, cit., p. 284.

39 Federalismo e libertà, cit., pp. 289-290. 
secondari, dai secondari ai terziari, attraverso successivi consigli sempre più ristretti, l'uno dentro l'altro come scatole cinesi, sino al vertice dello stato federale costituito conseguentemente da due organi supremi, il Consiglio federativo professionale e il Consiglio delle regioni.

Tralasciando l'esposizione della struttura del nuovo stato nei suoi diversi istituti, importa richiamare l'attenzione del lettore sulla principale idea direttiva di questo progetto costituzionale: la compresenza della rappresentanza politica, per la quale l'individuo è rappresentato nei suoi interessi generali, e della rappresentanza degli interessi o funzionale o organica, per la quale lo stesso individuo è rappresentato come membro di un gruppo particolare in cui esercita la sua funzione sociale specifi$\mathrm{ca}$, di operaio, contadino, commerciante, intellettuale ecc. Con questo progetto Trentin riprendeva, se pure senza riferirvisi direttamente, un dibattito che era stato già molto vivace dopo la prima guerra mondiale fra i più strenui difensori della rappresentanza politica, come Luigi Einaudi, e i sostenitori della rappresentanza degli interessi, almeno in parte come un istituto destinato a integrare opportunamente la rappresentanza politica, una riforma di cui si era fatto banditore un altro autorevole rappresentante del pensiero liberale in Italia, Francesco Ruffini, e che non ha avuto alcun seguito nella elaborazione della nuova costituzione italiana dove la rappresentanza degli interessi è stata relegata in un istituto secondario e nato morto, come il Consiglio nazionale dell'economia e del lavoro. Ciò che se mai differenzia il progetto di Trentin dalle diverse proposte di correggere la tradizionale rappresentanza politica con una assemblea rappresentante degli interessi è, a mio giudizio, il rovesciamento del rapporto fra le due forme di rappresentanza: Trentin accentua l'importanza e anche la novità della prima e sembra alla fine che gli prema soprattutto l'istituzione e la garanzia dell'autonomia dei gruppi d'interesse. Nell'art. 11 della Costituzione per la Francia presenta la «Fédération des institutions autonomes» come costituita da «branches d'activité» e quindi da "circonscriptions territoriales». Descrive prima le «institutions primaires», costituite da ogni comunità economica e morale che sia fonte autonoma d'attività sociale, e in secondo tempo l'organizzazione territoriale dello stato (comunale, cantonale, dipartimentale e regionale). Nel progetto di costituzione italiana, nel capitolo sulle «basi istituzionali dell'organizzazione federale", enumera al primo posto le sette branche fondamentali in cui viene ripartita l'attività sociale dell'individuo (l'agricoltura, l'industria, il commercio, l'artigianato, la cultura, la stampa, la vita spirituale). Anche nel capitolo sull'organizzazione della repubblica, in entrambi i progetti, il Consiglio federativo professionale è nominato prima del Consiglio delle Regioni. Ancor più esemplarmente, nel programma d'azione pubblicato in appendice al primo numero del giornale clandestino "Libérer et Fédérer», scritto nel momento in cui l'idea deve incarnarsi nell'azione, sotto il titolo Organizzazione dell'ordine federalista 
si parla esclusivamente dell'elezione diretta dei consigli d'impresa e dei consigli di contadini, e di tutte le istituzioni in cui si esprimono le altre diverse attività e funzioni collettive, e solo marginalmente della federazione di questi consigli in connessione con la federazione dei differenti consigli sul piano territoriale ${ }^{40}$.

12. Trascurando i particolari organizzativi, che lasciano l'impressione di quella macchinosità che è propria di un progetto scritto a tavolino, risulta ben chiaro che il proposito di Trentin era di ideare un sistema sociale che oltrepassasse nello stesso tempo tanto il solo collettivismo economico che genera dispotismo politico, come nell'Unione Sovietica, quanto il solo liberalismo politico che lascia prosperare il dispotismo economico, com'è avvenuto nelle democrazie capitalistiche.

Per quanto negli ultimi mesi trascorsi in Italia prima della morte egli fosse insofferente di alcune ambiguità del Partito d'azione ${ }^{41}$, cui pure aveva aderito, e per quanto la soluzione federalistica da lui proposta non fosse quella del Partito d'azione, il quale parlava più genericamente di «rivoluzione democratica» e non era, pur nelle sue più rigorose espressioni, né anticapitalista sino in fondo in economia né pluralista sino in fondo in politica, Trentin perseguì con il suo progetto di stato socialista in economia e democratico in politica, se pure con maggiore consequenzialità non priva di una vena utopistica che nei fondatori del Partito d'azione non c'era, lo stesso obiettivo finale che era quello di capovolgere diametralmente la soluzione che alla crisi avevano dato i regimi fascisti, capitalistici in economia e dispotici in politica. Era una proposta che andava nella stessa direzione, se pure componendo elementi affini in un complesso sistematico diverso, della «rivoluzione liberale» di Gobetti (lo stesso Trentin parla delle «nostre fatiche di rivoluzionari liberali» ${ }^{42}$ quando vuol contrapporre la rivoluzione da lui auspicata a quella sovietica) e del socialismo liberale di Carlo Rosselli. A differenza della proposta di Gobetti che era essenzialmente politica e non dottrinale, la proposta di Trentin era anche dottrinale. A differenza di quella di Rosselli che era stata in un primo tempo dottrinale, nell'opera scritta al confino, Socialisme libéral, e poi anche pratica negli anni della milizia nel movimento di Giustizia e Libertà sino alla morte, quella di Trentin si contraddistingueva negli assunti teorici e nelle conclusioni pratiche. Il punto di partenza della dottrina di Rosselli era in largo senso filosofico: il socialismo, per essere conciliabile con la libertà e con la tradizione del pensiero libera-

${ }^{40}$ Federalismo e libertà, cit., pp. 367-369.

${ }^{41}$ Se ne vedano le tracce nella lettera di Trentin a Lussu del 23 ottobre 1943, pubblicata dallo stesso Lussu in appendice al Profilo di Silvio Trentin, in S. Trentin, Scritti inediti, cit., p. 26.

${ }^{42}$ In Riflessioni sulla crisi e sulla rivoluzione, in Antifascismo e rivoluzione, cit., p. 215. 
le, deve abbandonare il marxismo. Il nucleo del progetto di un uomo come Trentin, che era stato un giurista ed aveva una cultura giuridica professionale che né Gobetti, pur laureato in giurisprudenza, né Rosselli avevano, è essenzialmente istituzionale. Lo stato federale che egli prima tratteggia e poi espone sin nei più minuti particolari viene concepito come un ordinamento capace di salvaguardare la libertà del cittadino e l'autonomia degli enti collettivi in un'economia collettivistica; in quanto tale diventa lo strumento principe della difesa del pluralismo senza il quale non vi è governo democratico in una società in cui l'economia collettivistica tende a produrre un potere politico monistico.

Nell'ambito della stessa corrente di pensiero, la specificità del progetto di Trentin può essere bene individuata nelle due parole che danno il titolo sia al saggio del 1942 sia al movimento della Resistenza francese da lui promosso (e al titolo del giornale che ne esprime le idee): liberare e federare. Mentre il socialismo liberale fa pensare a una riforma volta a socializzare i mezzi di produzione, che è la quintessenza del socialismo, da compiersi osservando le regole del governo democratico, il motto di Trentin suggerisce l'idea di una liberazione che deve essere insieme dal potere economico e dal potere politico, seguita da una unificazione degl'individui così liberati in uno stato democratico integrale quale solo può essere uno stato dei consigli sia degli enti professionali sia di quelli territoriali. Fa pensare ad un processo diverso in cui viene prima il momento dell'emancipazione e poi quello della riunificazione, un processo, seguendo il quale, l'Europa avrebbe potuto riacquistare «con la sua unità il suo primato spirituale e con questo la sua missione nel mondo civile» ${ }^{43}$.

13. L'Europa. Riprendendo in conclusione il tema dell'inizio, è certamente vero che il federalismo di Trentin è più interessato al problema dell'organizzazione interna dello stato che a quello della organizzazione esterna degli stati nei loro reciproci rapporti. Ma è ora di aggiungere che egli non dimenticò mai il problema internazionale ${ }^{44}$.

${ }_{43}$ Federalismo e libertà, cit., p. 321. Rosengarten riproduce un manifesto di «Libérer et Fédéren, dove i due termini sono concisamente illustrati: "Libérer la France de l'envahisseur qui l'opprime, de la dictature qui l'étouffe, du capitalisme qui l'écrase, de la pourriture, de la démagogie et du mensonge qui l'avilissent: et pour cela, libérer la collectivité nationale de la tutelle écrasante de l'État centralisateur et centralisé, fatalement porté à devenir totalitaire, de l'État Moloch, par qui le citoyen est constamment rabaissé à la condition de membre d'un troupeau, si ce n'est au rôle d'automate. Fédérer toutes les forces qui engendrent et entretiennent la vie du corps social et les intégrer de façon organique et vivante dans un ordre constitutionnel qui concilie le respect de leur autonomie avec la solidarité qu'exige leur organisation en communauté" (Silvio Trentin, cit., pp. 191-192).

${ }^{44}$ Già nella prima fase dell'esilio francese egli contribuì a formare un patto d'alleanza fra repubblicani italiani e spagnoli, il $1^{\circ}$ ottobre 1928, a Bordeaux, in cui tra gli altri impegni, vi era anche quello di giungere alla fondazione degli Stati Uniti d'Europa (così in Rosengarten, Silvio Trentin, cit., p. 97). 
Della esigenza di avviare il processo degli Stati Uniti d'Europa si trova traccia in numerosi suoi scritti. In un'opera del 1930, Antidémocratie, che fa parte del gruppo di scritti di polemica antifascista, le ultime pagine sono dedicate a denunciare la politica antieuropea del fascismo e a trarne la conseguenza che «se il fascismo è l'anti-Europa, l'Europa non può non essere l'antifascismo» ${ }^{45}$, e pertanto: "Bisogna scegliere tra il mito sorpassato dell'indipendenza sovrana di ogni stato e l'ideale immanente di una cooperazione sempre più stretta e armoniosa di tutti gli stati che si rifanno a una medesima civiltà» ${ }^{46}$. Nel capitolo finale di $\mathrm{La}$ Crise du Droit et de l'État, là dove proclama la rigenerazione attraverso il federalismo, prevede non solo la ristrutturazione dello stato su basi federative interne ma anche «l'integrazione sempre più organica di questo nell'ordine completo dello stato universale» ${ }^{47}$. In Stato Nazione Federalismo vi sono alcune pagine polemicamente molto aspre nei riguardi del falso europeismo di alcuni leader politici europei, come Churchill, Daladier, Attlee, mentre «i tempi sono maturi per una piena e spontanea fioritura della auspicata civiltà federalista», anche se «i pregiudizi nazionalistici non sembrano davvero del tutto espulsi dalla coscienza dei popoli anche i più evoluti» ${ }^{48}$. Come scrive nel saggio più volte citato, Liberare e Federa$r e$, è la stessa "logica del federalismo» che esige che il principio che esso pone a regola e misura necessarie delle relazioni della vita sociale venga applicato coerentemente e conseguentemente in tutte le sfere in cui la vita sociale si esplica. Sicché la Rivoluzione non avrà adempiuto il suo compito se non quando l'intera umanità avrà raggiunto la sua unificazione istituzionale in un'unica forma federale, e gli stati, completando il processo che è all'origine della loro formazione, potranno dissolversi nello stato $^{49}$. È in vista di questo sviluppo consequenziario del principio federativo che i due primi articoli dei progetti di costituzione francese e italiana recitano: «La France est membre fondateur des États Unis d 'Europe» e "L'Italia è una repubblica federale e rivendica la dignità e il titolo di membro fondatore della repubblica europea». Infine, nel programma d'azione del Movimento si legge: «Il governo rivoluzionario dovrà preparare, in collaborazione coi governi degli altri paesi liberati dal fascismo e dal nazismo, le basi di una federazione europea fondata sulla libertà, la pace e la prosperità,$^{50}$.

45 In Antifascismo e rivoluzione, cit., p. 52.

${ }^{46}$ Op. cit., p. 53.

${ }^{47}$ La crise du droit et de l'État, cit., p. 476; in Federalismo e libertà, cit., p. 29.

${ }^{48}$ Federalismo e libertà, cit., p. 205.

${ }^{49}$ Federalismo e libertà, cit., p. 290.

${ }^{50}$ Federalismo e libertà, cit., p. 369. 
Nell'ultimo scritto, l'Appello ai lavoratori delle Venezie, dopo aver ribadito il suo fermo ideale secondo cui «non vi può essere libertà senza autonomia», conclude: «Solo [così] può sorgere uno stato di uomini liberi ed eguali, e [ciò segnerà] il trionfo della Rivoluzione nel Continente europeo, e dunque la fine dello stato accentratore e dittatoriales ${ }^{51}$.

${ }^{51}$ Antifascismo e rivoluzione, cit., p. 538. 



\section{FRANÇOIS GÉNY*}

Quando cominciai i miei studi di filosofia del diritto, all'inizio del decennio tra il '30 e il '40, François Gény era all'apice della sua notorietà, soprattutto per le sue opere, Méthode d'interprétation et sources en droit privé positif di cui erano uscite due edizioni del 1899 e del 1919, e per l'opus magnum in quattro volumi Science et technique en droit privé positif, usciti uno dopo l'altro, nel 1914, 1915, 1921, e 1924. Orientato come allora verso studi di teoria della scienza giuridica e di metodologia in generale, che avevano molto seguito nella filosofia del diritto del tempo, entrambe le opere del giurista-filosofo francese mi erano familiari. Uno dei miei primi libri, oggi ripudiatissimo, anche se mi accade di vederlo qualche volta citato solo per completezza bibliografica da autori, che spero non l'abbiano letto, Scienza e tecnica del diritto (1934), mostrava chiaramente, se pur più nel titolo che nel contenuto, quale ne fosse l'ispirazione. Per quel che riguarda le tesi ivi sostenute, non solo la soluzione data da Gény alla distinzione tra scienza e tecnica fondata sulla distinzione fra il donné e il construit veniva messa completamente da parte, ma il giudizio che io davo in una nota sui presupposti filosofici da cui il celebre autore era partito, era, che Dio me lo perdoni, piuttosto malevolo, non privo di giovanile saccenteria. Del libro precedente sull'interpretazione mi servii ampiamente e senza i toni polemici in un'opera successiva su L'analogia nella logica del diritto (1938).

Fu con mia sorpresa che durante la preparazione di un discorso commemorativo che tenni all’Università di Venezia il 14 marzo 1954 su Silvio Trentin, che vi aveva insegnato tra il 1923 e il 1925, in occasione del decimo anniversario della morte ${ }^{1}$, appresi che dei molti libri che egli aveva

* François Gény: una prefazione, "Quaderni per la storia del pensiero giuridico moderno», 20, 1991, pp. 181-187.

${ }^{1}$ La prefazione di Gény non è sfuggita all'autore dell'unica monografia italiana su di lui: Aristide Tanzi, François Gény tra scienza e giurisprudenza, Torino, Giappichelli, 1990, p. 18, nota 8. L'autore fra l'altro constata che questa prefazione non è mai stata citata nella letteratura critica su Gény. [Il discorso di Bobbio su Trentin, compreso nel volume Italia Civile, I ed., Lacaita, Manduria 1964; II ed., Passigli, Firenze 1986, pp. 249-266, apre questo volume]. 
scritto in esilio in Francia, di tutte le sue opere quella filosoficamente più impegnativa, La Crise du Droit et de l'État, pubblicata a Parigi nel 1935, era preceduta da una lunga e ammirevole prefazione di François Gény, che definivo, così come era giusto, «uno dei più illustri giuristi francesi»².

Trentin, professore di diritto amministrativo e d'istituzioni di diritto pubblico, autore di vari scritti sui problemi dell'autogoverno locale e fautore di una politica di decentramento, era stato uno dei pochi professori che aveva scelto l'esilio piuttosto che la sottomissione al regime fascista ${ }^{3}$. Emigrò in Francia dove arrivò il 2 febbraio 1926. Nei primi anni vi fece l'agricoltore e il tipografo prima di trovare un assetto stabile nella conduzione di una libreria a Tolosa nel 1934. Nelle molte opere che scrisse in Francia, di cui la maggior parte sono di analisi e critica del fascismo, due sono di teoria giuridica e politica, attraverso cui torna a fare il professore universitario: quella già citata sulla crisi del diritto e dello stato e quella precedente su Les trasformations récentes du droit publique italien. De la Charte de Charles-Albert à la création de l'État fasciste, apparsa nel $1929^{4}$. Avrebbe dovuto essere presentata al pubblico francese da Maurice Hauriou, il celebre doyen della facoltà giuridica di Tolosa, se non fosse morto dopo breve malattia nel marzo dello stesso anno. Lo sostituì, meno celebre ma non meno noto, Julien Bonnecase dell'Università di Bordeaux, autore di due opere che fanno parte del patrimonio comune di uno storico del diritto moderno: L'école de l'exégèse en droit civil (II ediz. 1928) e Science $d u$ droit et romantisme (1928), che lo stesso Trentin dimostra di ben conoscere e di apprezzare.

Quale fosse il debito di riconoscenza che Trentin aveva contratto verso il Bonnecase è da lui stesso dichiarato nell'Avant-Propos al libro sulla crisi del diritto e dello stato, là dove esprime la sua riconoscenza per «l'obligeance fraternelle» dei colleghi delle facoltà di Bordeaux e di Tolosa e, in modo particolarmente caloroso, per l'«aide généreuse que n'a pas cessé un istant de m'offrir un témoignage d'une solidarité humaine émouvante, le professeur Julien Bonnecase, de la Faculté de droit de Bordeaux» ${ }^{5}$

2 Trattandosi di libro raro, ne riporto per intero il frontespizio: Silvio Trentin/ Ancien Professeur de Droit public dans les Facultés Italiennes/La Crise du Droit et de l'État/ Préface de F. Geny/ Doyen honoraire de la Faculté de Nancy/L'Eglantine/ParisBruxelles/1935, pp. 498.

${ }^{3}$ Sull'opera di Trentin, sino all'esilio in Francia, vedi Moreno Guerrato, Silvio Trentin, un democratico all'opposizione, Milano, Vangelista, 1981. Per uno studio monografico completo si veda Frank Rosengarten, Silvio Trentin dall'Intervento alla Resistenza, Milano, Feltrinelli, 1980: sulla prefazione di Gény, p. 140.

4 Tradotta in italiano a cura di A. Pizzorusso, nel primo volume delle «Opere Scelte di Silvio Trentin", Marsilio, Venezia 1983.

${ }^{5}$ S. Trentin, La Crise du Droit et de l'État, cit., p. 20. 
La Crise è dei libri di Trentin dell'esilio il più ambizioso e teoricamente importante, ma è anche quello meno fortunato. L'enorme mole (circa cinquecento pagine) e una meritoria ma anche un po' farraginosa rassegna della letteratura giuridica del tempo, lo rendono di non facile lettura: molto citato lo stesso Gény, ma anche Hauriou e Le Fur, Duguit e Saleilles, Bonnecase e Renard; tra gli italiani, Giorgio Del Vecchio e Santi Romano. Non mancano riferimenti a Kelsen, cui si ispira sin dall'inizio per la definizione di democrazia (di Kelsen era stata allora tradotta da Charles Eisenmann, La démocratie. Sa nature, sa valeur ${ }^{6}$ ). Il punto di riferimento principale è George Gurvitch, ammirato e criticato, i cui due libri fondamentali, Le temps présent et l'idée du droit social e L'idée du droit social erano entrambi usciti nel $1932^{7}$; nel suo insieme l'opera può essere oggi studiata per la parte che essa rappresenta nella fortuna dell'idea sociale in Francia.

Poco fortunata, dicevo, perché poco letta, introvabile nelle biblioteche italiane, e per la sua mole non ristampata, salvo la conclusione, nelle Opere scelte di Silvio Trentin, pubblicate, con introduzione di autori vari italiani contemporanei, da Marsilio tra il 1983 e il $1987^{8}$. Nell'evoluzione del pensiero di Trentin quest'opera è importante, perché è quella in cui appare per la prima volta proposta con determinazione l'idea del federalismo come soluzione della crisi del diritto e dello stato, conseguenza dello strapotere politico ed economico quale si è venuto formando in una società capitalistica, soffocante sempre più l'autonomia dei gruppi e calpestante la dignità della persona. Punto di partenza e punto di arrivo della complessa costruzione teorica del libro è la difesa della democrazia. Allo sviluppo e precisazione dell'idea federalistica cui giunge attraverso Proudhon e Gurvitch, che a sua volta ha ripreso Proudhon, saranno dedicate da Trentin le due principali opere successive, Libérer et Fédérer del $1943^{9}$ e Stato Nazione Federalismo del $1945^{10}$. Da sottolineare l'insistenza sull'importanza dell'associazionismo che caratterizza la società moderna, e sull'idea che solo lo stato federale permetta la convi-

${ }^{6}$ Sirey, Paris 1932.

7 Quando mi occupai di Gurvitch più o meno negli stessi anni ignoravo il libro di Trentin. Mi riferisco al mio scritto, Istituzione e diritto sociale (Renard e Gurvitch), in «Rivista internazionale di filosofia del diritto», XVI, 1936, pp. 385-418.

${ }^{8}$ Nel volume di scritti, già cit., Federalismo e libertà, col titolo La conclusione della crisi, pp. 4-31.

${ }_{9}$ Pubblicato per la prima volta nella traduzione italiana di Antonio Giuriolo in Silvio Trentin, Scritti inediti. Testimonianze e studi, Centro studi Piero Gobetti, Torino 1972, pp. 189-278, quindi nel volume Federalismo e libertà, cit., pp. 235-321.

${ }_{10}$ Scritto nel 1940, pubblicato postumo, con prefazione di Mario Dal Pra, nella «Biblioteca di cultura politica», n. 1, La fiaccola, Milano 1945. Il libro iniziava una collana edita a cura del Partito d'Azione cui Trentin aveva aderito dopo il ritorno dall'esilio nell'agosto 1943. Ora in Federalismo e libertà, cit., pp. 35-231. 
venza dei gruppi che tenderebbero, se lasciati liberi di esercitare il loro potere, a disgregare lo stato. Il federalismo è l'unica soluzione democratica tra autoritarismo dello stato sovrano e anarchia dei gruppi: «L'état ne peut s'organiser qu'en organisant la société, et la société, à son tour, ne peut être organisée selon le Droit qu'en formant son organisation à l'imperatif qui place la valeur suprême dans l'autonomie de la personne et dans celle dés ordres»" ${ }^{11}$.

Trentin ha colto benissimo il fenomeno caratteristico della società di un paese industrialmente progredito in quella che chiama la «pluralisation des ordres juridiques», e la tendenza verso il loro inserimento in sistemi sempre più vasti, destinato a trasformare lo stato futuro, se vorrà conservarsi democratico contro le spinte autoritarie del momento presente, in un "ordre d'intégration dans le droit des forces sociales", alla cui base sta «l'équivalence des autonomies, l'autonomie constituant la valeur suprême que le droit vise à servir» $»^{12}$. Non bisogna dimenticare che quando Trentin scrive quest'opera Hitler ha preso saldamente il potere in Germania, e nel 1934 in Francia in seguito a un'agitazione delle destre reazionarie il presidente del consiglio Daladier, che è un radicale, è costretto a dare le dimissioni e gli succede un governo di unità nazionale guidato da Doumergue, da cui sono esclusi socialisti e comunisti. Il fascismo, che nella interpretazione di sinistra, che Trentin segue, rappresenta la difesa ad oltranza del capitalismo minacciato dall'ineluttabile avanzata del movimento operaio, è ormai alle porte. La lotta per la democrazia e il federalismo che deve portare alla soluzione della crisi (Le dénouement de la crise è intitolato il capitolo conclusivo) non può essere che rivoluzionaria. L'emancipazione dell'individuo esige la soppressione dei privilegi e l'instaurazione di un ordine socialista. La rigenerazione della società contro lo stato moderno monocratico e monocentrico, non può avvenire se non "par le féderalisme en tant que mode de réalisation, sur le plan collectif, du principe de l'autonomie» ${ }^{13}$. A sostegno della tesi rivoluzionaria cita il famoso passo dei Discorsi di Machiavelli in cui si legge che le repubbliche meglio ordinate sono quelle che si possono rinnovare, perché «è cosa più chiara del sole che non si rinnovando questi corpi non durano ${ }^{14}$, e che per rinnovarsi debbono essere ricondotte ai loro principi. Per lo scopo che mi propongo in questa breve nota è interessante osservare che per giustificare la propria conclusione, Trentin cita anche un brano di Gény, uno dei suoi autori prediletti, là dove questi sostiene che quando la legge non riesce a controllare il potere politico e da so-

${ }^{11}$ S. Trentin, La Crise du Droit et de l'État, cit., p. 188.

12 S. Trentin, La Crise du Droit et de l'État, cit., p. 331.

13 S. Trentin, La Crise du Droit et de l'État, cit., p. 476.

${ }^{14}$ Il famoso brano dei Discorsi è tratto dal cap. I del libro III. 
vrana diventa suddita, il diritto si attua attraverso l'autodifesa: "Qu'on ne dise pas - s'écrie le Doyen Gény - : il y a contradiction dans les termes: le désordre ne peut etre ramené à l'ordre. Je répondrai que le prétendu désordre n'est ici que le cri de révolte d'un idéal plus parfait et qu'au témoignage de l'expérience, les progrès le plus décisifs de la civilisation et du droit ne sont acquis qu'au prix des révolutions $»^{15}$. Il libro termina con parole che non potrebbero essere più ispirate: «L'ère qu'annonce la catastrophe du capitalisme ne comptera dans l'histoire de la civilisation qu'en tant qu'elle saura marquer la révanche de l'autonomie humaine et la soumission des rapports sociaux qu'ils soient inter-individuels ou intergroupaus, à la seule souveraineté, à la souveraineté exclusive du Droit» ${ }^{16}$.

Trentin aveva scritto un'opera di teoria e insieme di proposta politi$\mathrm{ca}$, di riflessione sul proprio tempo e di proiezione ideale verso il futuro. Nella lunga prefazione, che non è un retorico rituale omaggio all'autore, ma contiene una analisi, una interpretazione, in parte critica, del libro presentato, Gény colse con profonda simpatia umana lo spirito del libro. Parla di «important ouvrage», di "profondes méditations, dont un dur éxile ne pouvait qu'augmenter la puissance», di «doctrine profonde», di «remarquable ouvrage», in cui l'autore fa valere le sue idee «douées souvent d'une profondeur impressionante et mises en un relief saisissant». È d'accordo sulle trasformazioni dello stato e del diritto che impongono una revisione delle categorie tradizionali. Distingue due parti o aspetti dell'opera, una prevalentemente teorica che risale ai principi dai quali si deve osservare e valutare la crisi, una orientata verso le possibili soluzioni della crisi. Questa seconda parte, Gény ritiene secondaria e contingente. Coglie bene quel che vi è di astratto nelle dottrine giuridiche e filosofiche, di cui l'autore si è largamente avvalso nel tracciare il disegno della situazione storica, per cui «si la direction générale de sa pensée reste assez aisément saisissable, elle laisse souvent subsister, autour d'elle et dans ses développements nécessaires, un halo vaporeux, qui ne permet pas toujours d'en fixer fermement les traits ${ }^{17}$. Riassumendone per sommi capi il contenuto, constata che l'ispirazione filosofica prevalente è spiritualistica, antipositivistica, favorevole al primato del diritto naturale sul diritto positivo. Ritiene particolarmente nuovo e fecondo il capitolo in cui lo stato è considerato essenzialmente come l'«ordine delle autonomie», tra diritto individuale e diritto sociale l'autore si pronuncia in favore del primo che salvaguarda la dignità umana, con un sentimento generoso che, diffuso in tutto il libro, arricchisce la nostra teoria generale del diritto e dello stato. Riguardo agli ultimi due capitoli in cui

\footnotetext{
15 S. Trentin, La Crise du Droit et de l'État, cit., p. 467.

${ }^{16}$ S. Trentin, La Crise du Droit et de l'État, cit., p. 478-479.

17 F. Gény, Avant-Propos, in S. Trentin, La Crise du Droit et de l'État, cit., p. 11.
} 
viene prendendo forma, se pure in modo talvolta confuso, il progetto politico di Trentin, il pur benevolo prefatore esprime, sebbene cortesemente, il proprio dissenso nei riguardi dell'«âpreté impitoyable» e dello «style heurté et parfois apocalyptique» con cui viene criticata la società borghese ${ }^{18}$. Riassunti i quattro punti principali della conclusione, dubita della validità del primo, che riguarda la necessità della rivoluzione, osservando che nessuna previsione scientifica ci permette di prevedere che il mutamento avvenga per gradi o attraverso un procedimento rivoluzionario. Critica il secondo che vede un avvio di soluzione nella critica del nazionalismo e nella formazione graduale dello stato universale. Considera formula vaga e troppo generale la proposta contenuta nel terzo punto, riguardante la soppressione della proprietà individuale, cui non sostituisce chiaramente il modello di una società fondata sulla proprietà collettiva. Per quello che riguarda il quarto punto, il progetto federalistico, lo considera semplice espressione di un desiderio che richiederebbe una precisa ricostruzione.

Nonostante queste riserve, nell'ultima pagina il vecchio saggio (nato nel 1861 quando scrive queste pagine ha $74 \mathrm{anni}$ ) esprime ancora una volta la propria ammirazione per l'indipendenza di giudizio dell'autore, «une admiration qui s'élève jusqu'à une émotion sympathique, qui ne peut qu'accroître ma reconnaissance, quand je songe aux conditions douloureuses et difficiles qu'un éxil, courageusement et noblement accepté, a imposées à la réalisation de cet effort». Nelle condizioni in cui è stata composta l'opera, l'autore diventa «un exemple sublime: exemple d'indépendance de pensée, d'énergie morale indomptable, de haute vertu civique, de fidélité, sans compromission, ni réserve, au pur idéal du Droit» ${ }^{19}$.

Non so se Silvio Trentin abbia ricevuto elogio più alto. So peraltro che tale elogio dimostra la nobiltà d'animo di chi l'ha pronunciato. Per questo ho creduto che meritasse di essere conosciuto nel momento in cui l'opera e la personalità dell'illustre doyen dell'Università di Nancy vengono riesaminate e riproposte al lettore di oggi.

${ }^{18}$ F. Gény, Avant-Propos, in S. Trentin, La Crise du Droit et de l'État, cit., p. 14.

19 F. Gény, Avant-Propos, in S. Trentin, La Crise du Droit et de l'État, cit., pp. 17-18. 
APPENDICE 



\title{
DOCUMENTI
}

\author{
LETTERE DI AGOSTINO ZANON DAL BO, \\ BEPPA NARDARI TRENTIN, ALDO GAROSCI, \\ BRUNO TRENTIN, NORBERTO BOBBIO
}

\author{
a cura di Pina Impagliazzo e Pietro Polito
}

I documenti pubblicati di seguito, conservati presso il Centro studi Piero Gobetti-Archivio Norberto Bobbio, unità archivistica ANB 1272, riguardano la partecipazione di Norberto Bobbio alla commemorazione di Silvio Trentin, tenutasi a Venezia nel decimo anniversario della morte, il 14 marzo 1954.

Dalla lettera di Agostino Zanon Dal Bo apprendiamo del progetto di edizione dell'opera di Trentin, Liberare e federare. Dare un senso e uno scopo alla rivoluzione europea in corso di gestazione, secondo la 'versione' eseguita da Antonio Giuriolo.

La moglie di Trentin, Beppa Nardari, accoglie con grande riconoscenza la notizia che sarà Bobbio, invitato da Enrico Opocher, a commemorare il marito: "La nostra gratitudine è immensa». Bruno Trentin «ne è veramente commosso» e pensa che il pensiero del padre "sarà spiegato» da Bobbio "come nessun altro potrebbe fare».

Sia Beppa sia Bruno sono prodighi di notizie e informazioni bibliografiche. Al riguardo Bobbio si rivolge ad Aldo Garosci che nella lettera del 3 marzo 1954 traccia sinteticamente un vero e proprio profilo di Trentin.

La lettera di Beppa successiva al ricordo di Trentin esprime soddisfazione e commozione. Ella scrive che Bobbio ha preparato la commemorazione «non soltanto con la sua rara intelligenza ma anche col suo cuore», chiarendo aspetti del pensiero di Trentin che "sembravano contraddittori», attraverso una "esposizione profonda sì ma semplice nello stesso tempo e alla portata di tutti».

A Bobbio rivolge riconoscente il ringraziamento di «aver dato a me e ai suoi figli che abbiamo tutto perduto, un immenso conforto e una grande dolcezza».

Il 28 marzo 1954 Bobbio così si rivolge alla Signora Beppa: «Non potevo desiderare premio più alto che queste sue parole». Poi aggiunge: "Quando scrivevo le pagine di quella commemorazione, sapevo che null'altro facevo se non compiere un piccolo dovere: quello di rendere testimonianza di chi ha dato, in sofferenze, sacrifici, infinitamente più di noi. Se non rendiamo questa testimonianza, l'essere noi vivi - dopo quella bufera e in questo presente deserto - ci parrebbe una colpa». 
1. Lettera di Agostino Zanon Dal Bo a Norberto Bobbio, 18-2-1954, su carta non intestata, dattiloscritto, con firma autografa: «tuo Agostino Zanon Dal Bo», 1 facciata. ANB 1272.

\section{Caro Bobbio}

forse ti ricordi ancora dell'opera inedita di Silvio Trentin di cui ti ho parlato l'ultima volta che ci siamo visti - a un convegno della SEC, a Venezia $^{2}-$. Eravamo rimasti intesi che te ne avrei inviato lo schema e gli estremi, perché tu ne potessi parlare all'editore Einaudi. Poi, un po' perché affaccendato in molte cose, un po' per certa mia inguaribile lentezza e pigrizia, non mi sono più fatto vivo. Ora la ricorrenza decennale della morte dell'autore e le insistenze del comitato per la celebrazione mi hanno "svegliato", e ti mando, acclusa, la serie dei titoli dei capitoli in cui l'opera è divisa.

Il manoscritto che io possiedo si compone di 96 cartelle dattilografate, a due spazi, e contiene la versione, eseguita da Giuriolo ${ }^{3}$, dell'ultimo lavoro di Silvio Trentin. Liberare e Federare ${ }^{4}$. La redazione francese, come sai, è andata perduta.

Il piano di pubblicazione che io proporrei è questo:

a) Prefazione di ...;

b) Introduzione del sottoscritto che dovrebbe contenere un rapido profilo del pensiero politico e dell'azione politica del Trentin;

c) Liberare e federare nella versione di Antonio Giuriolo;

d) L'abbozzo di costituzione federativa della Repubblica italiana che Trentin aveva steso durante il suo soggiorno in Italia ricalcandolo su un altro abbozzo ch'egli aveva già steso in Francia per la Repubblica francese;

e) I manifesti ch'egli aveva composto per il Gruppo regionale veneto del Partito d'Azione.

Il libro di meno di 100 pagine, comprenderebbe il momento ultimo dell'opera di Silvio Trentin, il momento costruttivo - in cui pensiero politico e azione erano diventato tutt'uno - interrotto dalla morte.

1 Agostino Zanon Dal Bo (Vittorio Veneto -Treviso, 1902-Venezia, 1993).

2 Terza riunione del Consiglio esecutivo della Società Europea di Cultura, Ca' Pisani, 4 ottobre 1954.

${ }^{3}$ Antonio Giuriolo (Arzignano, 1912-Lizzano in Belvedere, 1944). Cfr. N. Bobbio, Discorso su Antonio Giuriolo, in Id, Maestri e compagni, Passigli, Firenze 1984, pp. 189-202. Inoltre: R. Camurri (a cura di), I quaderni di Antonio Giuriolo, Marsilio, Padova 2016.

${ }^{4} \mathrm{~S}$. Trentin, Liberare e federare. Dare un senso e uno scopo alla rivoluzione europea in corso di gestazione, 1943. Il libro è stato scritto in francese, con il titolo di Libérer et Fédérer, nel 1942. Il testo tradotto da A. Giuriolo sul manoscritto trentiniano nel 1943 è riprodotto in Id., Scritti inediti. Testimonianze. Studi, contributi di E. Lussu e H. W. Tobler, a cura di P. Gobetti, Guanda, Parma 1972, pp. 187-278. e in Id., Federalismo e libertà. Scritti teorici 1935-1943, a cura di N. Bobbio, Marsilio, Venezia [gennaio] 1987, pp. 235-321. 
Esso non potrà uscire prima della commemorazione e nemmeno per la commemorazione, ma non sarebbe male che uscisse entro quest'anno. Sarebbe, del ricordo di Trentin, la parte più viva, dato che la celebrazione del mese prossimo va inesorabilmente assumendo carattere sempre più ufficiale (dico questo prescindendo, s'intende, dal carattere ben diverso da quello definito dall'aggettivo «ufficiale» che potranno avere i discorsi celebrativi).

S'intende che il mio studio introduttivo è un mio desiderio - legittimo, mi sembra, dati i miei rapporti col Trentin e il fatto che ritengo, purtroppo, d'essere uno dei pochi ancora convinti del valore politico effettuale della sua concezione federalistica -, ma non è affatto una condizione per la pubblicazione del manoscritto, che del resto non è cosa mia e che mi sta molto a cuore di veder comunque pubblicato.

Molti saluti cordiali Tuo Agostino Zanon Dal Bo Venezia, Cannaregio 2221A, 18/2/1954 
2. Lettera di Beppa Nardari Trentin a Norberto Bobbio, [dicembre 1953], su carta intestata: «1586, Campo San Giacomo dall'Orio, Venezia, tel. 25 187», manoscritto, con firma autografa: Beppa Trentin, due facciate fronte retro, ANB 1272.

Gentilissimo Professore,

il caro amico prof. Opocher ${ }^{5}$ ci scrive che lei ha accettato di commemorare mio Marito ${ }^{6}$ nel decimo anniversario della sua morte. Non so dirle, professore, quanto io e i miei figliuoli le siamo grati di questo. Bruno ${ }^{7}$ che ho visto qui per Natale ne è veramente commosso e mi incarica di dirle che nessuno meglio di lei potrà parlare di suo Padre ${ }^{8}$, così come egli avrebbe voluto. So che questo le costerà del lavoro e fin d'ora la ringrazio di tutto e le chiedo scusa.

Negli "scritti clandestini" di Egidio Meneghetti ella troverà un riassunto della vita di Silvio, molto esatto. Io le aggiungo la lista dei suoi scritti che lei in parte già conosce:

Del 1909: Sull'impugnativa in via possessoria degli atti amministrativi ${ }^{10}$. Del 1910: Responsabilità Collegiale - con prefazione di Giovanni Vacchelli.

Del 1920 o 21: L'atto amministrativo.

Del 1910: La cosa giudicata.

Del 1925: Autonomia - Autarchia - Decentramento.

Poi, l'esilio - e allora scrisse:

L'aventure italienne 1926.

La Crise du Droit et de l'État.

${ }^{5}$ Enrico Opocher (Treviso, 1914-Padova, 2004).

${ }^{6}$ Silvio Trentin. Per le notizie relative ai familiari di Silvio Trentin, si veda qui la nota sulla famiglia, a cura di Luisa Bellina.

${ }^{7}$ Bruno Trentin (Pavie, 1926-Roma 2007).

${ }^{8}$ Silvio Trentin (San Donà di Piave, 1885-Monastier di Treviso, 1944).

${ }^{9}$ Egidio Meneghetti (Verona, 1892-Padova, 1961). Cfr. Id., Scritti clandestini, con quattro xilografie di A. Sartori, Zanocco. Padova 1946, pp. 169. Prima edizione in carta di lusso numerata dall'1 al 500. Finito di stampare coi tipi dello stampatore Zanocco il 15 settembre 1945. Collana della cospirazione, 1. I titoli successivi previsti nella collana: C. Marchesi, (titolo da definire); S. Trentin, Ultimi scritti; A. Zamboni, Comitato di Liberazione Nazionale; S. Boscardi, Palazzo Giusti. Si veda: N. Bobbio, Egidio Meneghetti, «Il Ponte», XVIII (3), marzo 1961, pp. 316-319; Id., Egidio Meneghetti, «Resistenza», XV (3), marzo 1961, p. 7; Id. Commemorazione di Egidio Meneghetti, "Annuario dell’Università di Padova", a.a. 1984-1985, Società cooperativa tipografica, Padova 1985, pp. 5-15. Il discorso del 1985 è compreso in N. Bobbio, La mia Italia, a cura di P. Polito, Passigli, Firenze 2001, pp. 237-253. Inoltre: C. Savona, Egidio Meneghetti. Scienziato e patriota, combattente per la libertà, Istituto veneto per la storia della Resistenza e dell'età contemporanea, CLEUP, Padova 2003, che tra i documenti, alle pp. 240-243, propone: E. Meneghetti, Ricordo di Trentin, "Fratelli d'Italia», 10, 15 maggio 1944.

${ }^{10}$ Per le indicazioni complete delle opere di Silvio Trentin citate di seguito si veda la bibliografia pubblicata in questo volume, a cura di Pina Impagliazzo e Pietro Polito. 
Les transformations du droit public italien.

Riflessioni sulla crisi e sulla rivoluzione.

Antidémocratie.

Le fascisme a Genève.

Dalla bonifica integrale al "pane dell'Impero".

10 ans de fascisme totalitaire (questo libro non esiste più poiché essendo stato stampato dalle "Presses Unniversitaires" a Parigi, i tedeschi ne ordinarono la distruzione).

Stato Nazione Federalismo del 1943.

Bruno mi ha detto che tutti questi libri si trovano alla "Fondazione Gramsci" a Torino presso il dottor Carbone che lei conosce. Silvio scrisse anche un piccolo libro su Leopardi ${ }^{11}$ e uno su Lauro De Bosis ${ }^{12}$. Io sono a sua completa disposizione, Professore, per quanto occorrerle - per quanto può occorrerle - e grazie ancora infinite. Auguri carissimi per il nuovo anno da me e dai figliuoli - sua Beppa Trentin.

${ }^{11}$ S. Trentin, D'un poète qui nous permettra de retrouver l'Italie, Giacomo Leopardi, Stock, Paris 1940, pp. 118.

${ }^{12}$ Lauro De Bosis (Roma, 1901-Mar Tirreno, 1931). Cfr. S. Trentin, Lauro De Bosis, chantre et héros de la liberté, Commemorazione tenuta a Tolosa su iniziativa del "Cercle d'études universitaires", il 19 dicembre 1937, Jean Flory, Paris 1939, pp. 95. 
3. Lettera di Beppa Nardari Trentin a Norberto Bobbio, [precedente al 14 marzo 1954], su carta intestata: «1586, Campo San Giacomo dall'Orio, Venezia, tel. 25 - 187", manoscritto, con firma autografa: Beppa Trentin, due facciate fronte retro, $A N B 3704$.

Gentilissimo Professore,

eccole una copia dello scritto di Meneghetti ${ }^{13}$ e uno di Emilio Lussu ${ }^{14}$. Sono a sua disposizione per tutto quanto può occorrerle e la prego di non avere nessuno scrupolo. Siamo così contenti tutti che sia Lei a commemorare il nostro caro! - ne ho riparlato con Bruno che è passato di qua per poche ore di ritorno dalla Francia - mi ripeteva anche lui la sua soddisfazione e la sua commozione. Bruno ha molta ammirazione per lei, Professore e sa che il pensiero di suo Padre sarà spiegato da lei come nessun altro potrebbe fare. La nostra gratitudine è immensa. Lei parlerà, Professore, all'Università di Ca' Foscari dove sarà scoperta una lapide. La data non è ancora fissata ma sarà certamente o il $12 \mathrm{o}$ il $13 \mathrm{o}$ il 14. Appena la commissione avrà deciso il giorno, le scriverò anche per sapere se tale giorno è di sua convenienza o no.

Grazie di tutto. Se vede Franco Venturi ${ }^{15}$ lo saluti tanto tanto per me. A lei da me e da Giorgio ${ }^{16}$ una affettuosa stretta di mano, sua Beppa Trentin

13 Dattiloscritto, con intestazione autografa: Egidio Meneghetti: apparso nel foglio clandestino: Fratelli d'Italia, fogli 7 (ANB 1272). E. Meneghetti, Ricordo di Silvio Trentin, in Id., Scritti clandestini, pp. 39-49.

${ }^{14}$ Emilio Lussu (Armungia, 4 dicembre 1890-Roma, 5 marzo 1975). Cfr. Dattiloscritto, con intestazione autografa: Emilio Lussu in 'Giustizia e Libertà, fogli 4. In calce al testo: «Commemorazione fatta da Emilio Lussu a Roma nel 1944».

${ }^{15}$ Franco Venturi (Roma 1914-Torino 1994).

16 Giorgio Trentin (San Donà di Piave, 1917-Venezia, 2013). 
4. Lettera di Bruno Trentin a Norberto Bobbio, senza data [ma precedente al 14 marzo 1954], su carta non intestata, dattiloscritto, con firma autografa: Bruno Trentin, due facciate, ANB 1272

Caro professore, ho spedito ieri "Riflessioni sulla crisi e sulla rivoluzione" che era in mio possesso. Mi sto interessando presso la Fondazione Gramsci perché Le sia spedito al più presto, "La crise du droit et de l'État".

Per quanto riguarda i dati che Ella mi chiede spero che le seguenti indicazioni potranno esserLe utili.

Mio padre prese la Libera docenza nel 1910, con un'opera dal titolo: "La cosa giudicata nelle decisioni giurisdizionali delle Sezione del Consiglio di stato". Il suo maestro era Giovanni Vacchelli17.

Successivamente egli insegnò alla Università di Camerino per un anno circa, e nel 1911 andò a Heidelberg, dove rimase fino al 1914, come lettore all'Università. Lì, scrisse: "L'atto amministrativo".

Alla fine della guerra insegnò a Padova, nel 1919, poi a Camerino, poi a Macerata e finalmente a Venezia, dove fu insediato in cattedra nel 1924. Egli era titolare di Diritto Amministrativo, ma a Venezia insegnò Diritto Pubblico e Internazionale.

Partecipò al Movimento dei Combattenti per il Rinnovamento e fu eletto Deputato nel 1919 quale esponente di quel Movimento. Con la sua scomparsa egli passò alla socialdemocrazia. Si occupò attivamente della ricostruzione delle "terre liberate" e fu tra i promotori dell'Istituto Federale di Credito delle Tre Venezie.

Nel 1919, a mia conoscenza, fu inviato a Fiume come plenipotenziario per trattare la resa. Ma non credo che la cosa rivesta qui molta importanza.

Penso che, per quanto riguarda il periodo francese e il ritorno di mio padre in Italia, Ella sia sufficientemente informato.

Rimango comunque a sua completa disposizione per qualsiasi informazione $\mathrm{o}$ documentazione che Le potrà occorrerLe.

Vorrei inoltre, Caro Professore, esprimerLe tutta la mia gratitudine per quello che Lei fa e testimoniarLe la mia profonda emozione nel sapere che Ella ha voluto assumersi questo incarico.

Mi creda sempre Suo

Bruno Trentin

17 Giovanni Vacchelli (Cremona. 1866-Milano, 1960). 
5. Lettera di Aldo Garosci a Norberto Bobbio, Roma, 3 marzo [1954], su carta non intestata, dattiloscritto, con firma autografa: Aldo Garosci, tre facciate, ANB 1272

Roma, 3 marzo,

Caro Bobbio,

spero di rimandarti tra due o tre giorni la bibliografia completa. È stato un lavoro non semplice, in quanto molti dei libri da me citati non si trovano, almeno nelle edizioni originali, nelle biblioteche italiane, e ho dovuto ricorrere ad amici. Per i pochi dati che mi mancano tuttora spero provvederà Silone. Le bozze le ho riviste fin da principio; esse sono notevolmente corrette, sia detto a lode dello stampatore e dell'editore. Spero che quest'ulteriore ritardo non sarà di danno al volume

Ed eccoci ora quel che so di Trentin e del suo socialismo. Trentin, come bene hai visto leggendo le cose sue, non era originariamente un socialista ma uno di quei democratici radicali, piuttosto logici e consequenziari, per i quali il passaggio almeno a un certo tipo di socialismo (giacobinismo egualitario) può intellettualmente giustificarsi appieno. Era di ricca famiglia, proprietario terriero, combattente, massone, (ma non era colpa sua) cugino del famigerato Vittorio Cian ${ }^{18}$. Quando egli andò in Francia la sua posizione morale e materiale era ancora quella avuta in Italia; là egli intendeva sistemarsi acquistando terre nel mezzogiorno e dirigendole personalmente. Tale impresa gli andò fallita, ebbe gravi perdite finanziarie, e durante un tempo abbastanza lungo fece, per vivere, o almeno per guadagnare durante un periodo difficile, l'operaio tipografo. Fu in questo momento che cominciarono a manifestarsi le sue tendenze socialiste. A che dovute? Non saprei dirtelo, perché anche io arrivai in Francia in un periodo ulteriore (1932) e da quando giunsi a Parigi sentii sempre parlare di lui come nella posizione che ho detta, quantunque nessuno ignorasse la sua precedente posizione puramente democratica. Possono aver contribuito a questa evoluzione molti fattori:l'ambiente tolosano, dove per essere mediamente progressivi e soprattutto antifascisti senza esitazione bisognava essere socialisti; le sue proprie esperienze di vita operaia; (quantunque non avesse acquistato neppure in minima parte la psicologia operaia); il crescere della tensione e le infelici prove degli Stati democratici di fronte all'avanzare del fascismo. Tutti i conti fatti mi sembra anzi che questo sia stato il fattore determinante; tanto più che, fin da quando entrai in relazione con Trentin (più o meno, dalla costituzione di G.L. come gruppo indipendente, cioè dal 1934 in poi) a questa sua ideologia socialista corrispondeva non simpatia per i socialisti ma per i comunisti; i socialisti avevano gli stessi peccati di debolezza e compromesso degli altri democratici, e non è escluso anzi che nella sua nuova posizione (come in quella di molti ex democratici) entrasse per l'appunto un po' del suo disprezzo per i socialisti tradizionali. Su questo punto la sua posizione non mi sembra molto diversa da quella di Lussu, quantunque questi, fondandosi anche su frasi di Gramsci - che in ciò vedeva con gli occhi d'un sardo - abbia mitologizzato come socialista la sua, posizione di sardista.

18 Vittorio Cian (San Donà di Piave, 1862-Ceres, 1951). 
Sta di fatto: a) che Trentin ebbe sempre posizioni di fronte popolare più spiccate di tutti noi, tanto che aderì (nel '36) personalmente all'Unione Popolare, alla quale mai aveva aderito G.L., per quanto, per disciplina di gruppo e solidarietà con noi non vi assumesse mai nessuna carica; b) che sentimentalmente rimase sempre molto legato al suo passato democratico: nella cantina-studio della sua libreria di Tolosa (dopo l'esperienza operaia s'era fatto libraio) egli teneva le fotografie di molti uomini politici democratici, con dedica, non solo di Amendola ${ }^{19}$ e $\mathrm{Nitti}^{20}$, ma anche di minori (che adesso non ricordo, ma che potrei situare tra Gasparotto e Luzzatti ${ }^{21}$, quantunque possa anche trattarsi di nessuno dei due). Ebbe uno choc assai violento, come del resto tutti noi, dal patto germanosovietico e fu a quell'epoca che scrisse il suo libro socialista-federalista; ma presto tornò al frontepopolarismo, come risulta anche dalla dichiarazione di alleanza a tre sottoscritta con Amendola ${ }^{22}$ e $\mathrm{Nenni}^{23}$ (nel '43 credo).

Questo è ciò che so dell'attività di Trentin. Devo aggiungere una cosa? [sic]. E che cioè il suo temperamento - leale, diritto, conseguente - mi parve sempre superiore alla capacità reale sua di vero e proprio pensiero teorico-speculativo [sic] Anche in questo, come socialista classista o come proudhoniano, egli rimase sempre il democratico giacobino di prima, fornito di chiarezza giuridica, nonché di eloquenza letteraria, e anche tormentato se si vuole, ma non di profondità speculativa e neppure di profondo senso di ciò che è nuovo nella situazione politica, e che sfugge quindi alla classificazione. In questo senso, egli era più simile (nella diversità del temperamento o delle qualità di scrittore) a un Lussu e magari a un Cianca ${ }^{24}$ che a un Rosselli ${ }^{25}$ (da cui pure alcuni tratti di cultura del democratico tradizionale, del radicale alla Salvemini ${ }^{26}$ non erano assenti).

Non so se ti ho detto nulla di veramente utile. Se la moglie ha conservato lettere o corrispondenze, non dovrebbe essere difficile trovarvi, tra il 1929 e il 1932, documenti della crisi a cui accenno.

Affettuosi saluti.

Aldo Garosci ${ }^{27}$

Ps. Ho saputo dei concorsi per "dottrine politiche" e "storia moderna". Quali saranno i termini utili per presentare i titoli, da quel che puoi prevedere?

19 Giovanni Amendola (Salerno, 1882-Cannes, 1926).

${ }^{20}$ Francesco Saverio Nitti (Melfi, 19 luglio 1868-Roma, 20 febbraio 1953).

${ }^{21}$ Luigi Luzzatti (Venezia, 1841-Roma, 1927).

22 rivoluzionario Giorgio Amendola (Roma, 21 novembre 1907-5 giugno 1980).

${ }^{23}$ Piero Nenni (Faenza, 1891-Roma, 1988).

${ }^{24}$ Alberto Cianca (Roma, 1984-Roma, 1966).

${ }_{25}$ Carlo Rosselli (Roma, 1899-Bagnoles de l'Orne, Alençon 1937).

26 Gaetani Salvemini (Molfetta, 1873-Sorrento, 1957).

27 Aldo Garosci (Mena di Susa-Torino, 1907-Roma 2000). Cfr. D. Pipitone, Alla ricerca della libertà. Vita di Aldo Garosci, FrancoAngeli, Milano 2017. 
6. Lettera di Beppa Nardari Trentin a Norberto Bobbio, [successiva al 14 marzo 1954], su carta intestata: «1586, Campo San Giacomo dall'Orio, Venezia, tel. 25 - 187', manoscritto, con firma autografa: Beppa Trentin, due facciate fronte retro, $A N B 3704$.

Caro Professore e amico,

Franca ${ }^{28}$ e Bruno sono ripartiti per Perugia e Roma martedì sera - nei due giorni passati ancora qui con me non hanno fatto che parlare di lei - . Tutti uniti abbiamo riflettuto, ripensato, tentato di ricordare quasi frase per frase la sua magnifica commemorazione - e, con altri amici, con tutti gli amici che abbiamo visto dopo, abbiamo concluso che non poteva essere né più bella né più degna. Come dice giustamente Bruno, si pensa con rispetto al lavoro profondo che lei ha fatto per il nostro caro e per noi - noi abbiamo sentito che lei ha preparato questa commemorazione non soltanto con la sua rara intelligenza ma anche col suo cuore. Certi aspetti del pensiero di Silvio che io non avevo capito e che mi sembravano contraddittori, mi sembrano ora così chiari grazie alla sua esposizione profonda sì ma semplice nello stesso tempo e alla portata di tutti. Ero così sconvolta domenica che non ho saputo dirle bene la mia gratitudine - vorrei che ora, tornato nella sua casa vicino alla sua signora e ai suoi figliuoli, ella potesse sentire una grande soddisfazione: quella di aver dato a me e ai suoi figli che abbiamo tutto perduto, un immenso conforto e una grande dolcezza. Si ricordi professore che la nostra casa le è sempre aperta e che sarà per noi un onore e una gioia poterla ospitare. Grazie con tutto il cuore sua Beppa Trentin.

${ }^{28}$ Franca Trentin (Venezia, 1919-Venezia, 1920). 
7.Lettera di Norberto Bobbio a Beppa Nardari Trentin, con data: "Torino, 28.3.54", su carta intestata: "Università degli studi di Torino", manoscritto, con firma autografa: "Norberto Bobbio". Fondo Franca Trentin Archivio rEsistenze, Casa della Memoria, Venezia.

Torino, 28. 3.54

Cara e Gentile Signora, la ringrazio della sua lettera. Le sue parole, che sono anche quelle dei suoi figli, mi hanno commosso. Non potevo desiderare premio più alto che queste sue parole. Alle quali si è aggiunto il bellissimo ritratto di suo marito, che avevo già visto riprodotto altre volte: anche questo $\mathrm{e}$ una testimonianza, che io serberò preziosissima, della nostra amicizia, alla quale tengo e che ricambio, con devota ammirazione, per lei, e con affetto, per i suoi figli. Quando scrivevo le pagine di quella commemorazione, sapevo che null'altro facevo se non compiere un piccolo dovere: quello di rendere testimonianza di chi ha dato, in sofferenze, sacrifici, infinitamente più di noi. Se non rendiamo questa testimonianza, l'essere noi vivi - dopo quella bufera e in questo presente deserto - ci parrebbe una colpa.

Chissà che debba venire a Venezia per la Società Europea di Cultura quest'estate, e che riesca a portarvi anche mia moglie. Sarei tanto lieto di poterla rivedere. E intanto le rendo l'omaggio di ammirazione e di affetto, che le dobbiamo, per la devozione con cui ha partecipato a quella nobile e sventurata esistenza, e per la fedeltà commovente con cui ne custodisce la memoria, e contribuisce a tramandare.

Con devoti e affettuosi saluti,

Norberto Bobbio

P.S. Ho ricevuto il testo della commemorazione e l'ho spedito a Calamandrei 



\title{
SILVIO TRENTIN E LA FAMIGLIA
}

\author{
Luisa Bellina
}

Silvio Trentin nasce nel 1885 a S. Donà di Piave da una agiata famiglia di proprietari terrieri. Compie studi di giurisprudenza a Pisa sotto la guida di Giovanni Vacchelli, ma maturando una personale «visione realistica e umanistica del diritto» ${ }^{1}$. Fortemente influenzato da una solida cultura illuministica e dal pensiero costruttore di Carlo Cattaneo, nei suoi primi studi scientifici indaga il tema della natura giuridica dei consorzi di bonifica e dello stretto legame tra lo sviluppo agrario e il miglioramento delle condizioni igieniche nelle campagne.

Nel 1910 ottiene la libera docenza in diritto amministrativo a Pisa, l'ordinariato nel 1913 presso la libera Università di Camerino; tra il 1913 e il 1914 frequenta un corso di perfezionamento all'università di Heidelberg con il giurista svizzero F. Fleiner.

Si arruola volontario nella Grande Guerra con il grado di sottotenente in qualità di amministratore della Croce Rossa, poi, per sua insistita pressione, nell'ultimo anno di guerra è ammesso in un'arma combattente come pilota mitragliere e ricognitore con la qualifica di tenente aviatore della $131^{\wedge}$ Squadriglia della Terza Armata dedicata ai servizi informativi, dislocata nelle vicinanze di Venezia nei campi di Marcon e Tessera. L'aeronautica è il suo mito, elemento eroico romantico - il gusto del rischio e dell'avventura l'hanno contrassegnato fin dall'infanzia - e nel contempo prospettiva di modernità tecnologica. Partecipa ad azioni audaci che gli varranno encomi e decorazioni.

Nel 1919 si trasferisce con la famiglia - la giovane moglie Giuseppina (Beppa) Nardari (Treviso, 1892) sposata nel 1916 e il figlio Giorgio (San Donà di Piave, 1917) - a Venezia dove nasce la secondogenita Franca. Inizialmente egli si colloca all'interno del combattentismo e dopo una breve attrazione per il messaggio di rinnovamento radicale del fascismo, alle elezioni politiche nel novembre dello stesso anno è eletto deputato nelle file della Democrazia sociale veneta, una magmatica formazione

${ }^{1}$ F. Cortese, Silvio Trentin. Maestro di diritto, apostolo della democrazia, pubblicazione a cura del Centro Documentazione e Ricerca Trentin, 2012, <http://www.centrotrentin. it/testi-e-materiali-online/articoli.html> (12/2019). 
democratico-riformista comprendente anche esponenti dei combattenti e del Fascio Veneziano. Anche se di breve durata per la fine anticipata della legislatura nel 1921, l'esperienza parlamentare di Trentin, sempre più orientato a favore di una politica democratica non conservatrice, si contraddistingue per un forte sostegno all'istituzione della Commissione parlamentare d'inchiesta sulle terre liberate e redente e dell'Ente di rinascita agraria delle province di Venezia e Treviso e all'approvazione del decreto che autorizza la bonifica integrale del terreni paludosi tra $i$ fiumi Lemene e Livenza. Dopo la sconfitta alle elezioni politiche del '21 continua ad impegnarsi in intense battaglie civili nel Veneto minacciato nuovamente dalla malaria, sui problemi della rinascita dopo le distruzioni della guerra, la bonifica delle terre come forma di redenzione umana, la sorte delle piccole industrie, la realizzazione dell'Istituto federale di credito per il risorgimento delle Venezie, il ruolo degli enti pubblici di fronte al problema delle utilizzazioni idrotecniche e al tentativo di monopolio delle risorse idriche da parte della società elettrica Sade.

Dal 1922 riprende l'insegnamento come professore straordinario di diritto amministrativo all'Università regia di Macerata. Dal '24 è professore straordinario di diritto pubblico e diritto internazionale al Regio Istituto superiore di commercio e di scienze economiche di Ca' Foscari a Venezia.

In particolare dopo l'assassinio Matteotti la sua opposizione al fascismo diviene radicale. Aderisce all'Unione nazionale fondata da Giovanni Amendola; nel 1925 si iscrive al Partito repubblicano, firma il manifesto degli intellettuali di Croce. Le intimidazioni fasciste nei suoi confronti si intensificano.

Nel gennaio del 1926, in seguito al Decreto-Legge 24 dicembre 1925 n. 2300 che obbliga tutti i funzionari statali all'obbedienza anche intellettuale e scientifica al regime fascista, decide di dare le dimissioni da docente, con una lettera al rettore in cui asserisce di non poter sottostare a doveri che aveva il «dubbio (quasi la certezza) di non saper conciliare con il rispetto delle [sue] più intime e più salde convinzioni di studioso di diritto pubblico». $\grave{E}$ in quel momento una scelta controcorrente, condivisa nell'intero corpo accademico italiano solo da altri due colleghi, Salvemini e Nitti; ed è anche una scelta di vita che coinvolge l'intera famiglia: a fine gennaio 1926 il brillante quarantenne avvocato e professore universitario espatria con moglie e figli in Francia, lasciandosi alle spalle la tranquillità di una vita personale e famigliare protetta dall'agiatezza economica, il prestigio sociale e i privilegi professionali.

Vende tutte le sue proprietà e acquista una tenuta agricola a Pavie, nel sud-ovest della Francia, che però, nonostante le moderne tecniche agronomiche adottate, rapidamente fallisce a causa della crisi monetaria in corso. Malgrado le preoccupazioni economiche riesce ad abbinare un'intensa attività pubblicistica e di ricerca all'impegno politico nell'or- 
bita della Concentrazione antifascista; aderisce al partito repubblicano francese e collabora attivamente con la Lega dei diritti dell'uomo.

Nel '28, perduti i propri averi, la famiglia - nel dicembre del '26 è intanto nato il terzogenito, Bruno - si trasferisce in un modesto appartamento nella cittadina di Auch, dove Silvio trova un lavoro come semplice operaio in una tipografia. Questa da lui stesso definita "proletarizzazione forzata» si rivela una esperienza decisiva di revisione politica e anche esistenziale. In quegli anni rimane incessante l'attività pubblicistica sia come momento di battaglia politica che come studio scientifico. Dall'interpretazione delle origini del fenomeno fascista dei primi scritti dal '28 al '31 (L'aventure italienne, Les transformations récentes du droit public italien, Aux sources du fascisme, Antidémocratie), allargando via via l'analisi a una visione della crisi giuridica e istituzionale democratica nell'Europa postbellica e al rapporto tra democrazia, Stato liberale e fascismo, il suo interesse è ora rivolto alla ricerca di una via per ricostruire la società attorno al valore supremo della libertà individuale, non astratto ma nel suo potere di realizzazione, in un nuovo ordinamento federale che armonizzi economia socialistica e politica democratica (Riflessioni sulla crisi e sulla rivoluzione, 1933, La Crise du Droit et de l'État, 1935).

Dal 1929 entra a far parte di Giustizia e Libertà, divenendo con Lussu autorevole esponente dell'ala sinistra. Suoi interlocutori in quegli anni sono - oltre allo stesso Emilio Lussu - Carlo Rosselli, Alberto Tarchiani, Francesco Volterra, Gaetano Salvemini, nonché Luigi Campolonghi, Francesco Ciccotti, Fernando Schiavetti, Filippo Turati ed Egidio Reale. La sua militanza si contraddistingue per un bisogno a volte febbrile di azione, in cui si uniscono rigore intellettuale e morale, utopia e senso forte e pragmatico della realtà concreta.

Nel 1934 Silvio è licenziato dalla tipografia e la famiglia si ritrova in gravi condizioni economiche. Grazie ad aiuti di parenti e amici acquisiscono una piccola libreria a Tolosa dove si trasferiscono nel '35. La Librairie du Languedoc, benché minuscola, diventa in poco tempo «un des grands foyers pensants de Toulouse», luogo di ritrovo di professori, avvocati, magistrati, politici francesi oltre che dei rifugiati italiani. Trentin fu un «un esule diverso dai più», scrive Paladini, non rinchiuso dentro la logica dell'emigrato impegnato a continuare, in altre forme, la battaglia di prima, bensì, fattosi culturalmente francese, un europeo capace di dialogare con la storia del suo tempo ${ }^{2}$.

L'intransigenza morale, il gusto dell'azione concreta, l'europeismo esistenziale come stile e etica di vita sono alla base anche dell'educazione dei figli. Nel 1936 la guerra di Spagna coinvolge tutta la famiglia e

${ }^{2}$ G. Paladini, "Figlio del Veneto": colloqui parigini su Trentin fra esilio e Resistenza, «Venetica», 3, gennaio-giugno 1985, p. 85. 
sarà una tappa decisiva nella formazione politica dei figli Trentin, nella visione internazionale delle cose che accompagnerà sempre ognuno di loro, nei distinti percorsi umani e professionali. Tolosa è una città di passaggio obbligato per chi va o viene dal fronte spagnolo. La libreria e la casa Trentin diventano il centro di smistamento dei volontari dalle diverse nazionalità e lingue, di spedizione di armi, una sorta d'ambasciata per i collegamenti irregolari fra la Francia e Barcellona. Silvio stesso si reca almeno quattro volte in zona di guerra. Dopo la sconfitta la libreria e la casa ridiventano luoghi di passaggio all'incontrario. Arrivano anche gli esuli spagnoli in fuga da Franco, che Beppa con i figli assiste nei campi profughi.

Il continuo crescendo di fatti ed esperienze dolorose compatta sempre più la famiglia. L'assassinio dei fratelli Rosselli il 9 giugno '37 è vissuto come un lutto famigliare, oltre che come grande perdita politica. Trentin urla «il [suo] orrore... il [suo] odio mortale» in un epico discorso nella Salle des Jacobins a Tolosa davanti a 20.000 persone.

Con lo scoppio della guerra mondiale la famiglia (in particolare Giorgio e Franca, mentre l'enfant terrible Bruno non ancora sedicenne è impegnato con il suo gruppo di liceali anarchici) è fattivamente coinvolta nelle iniziative di Silvio per organizzare la resistenza e la rete di collegamento tra servizi inglesi e resistenti francesi. Per riscattare la honte della dichiarazione di guerra dell'Italia alla Francia, Silvio alla radio dichiara a nome dei rifugiati italiani la fedeltà alla repubblica francese e con il figlio Giorgio chiede di essere arruolato nell'esercito francese, cosa che si rivelerà impossibile in quanto privi della cittadinanza. In libreria passa il variegato mondo di studenti, intellettuali, artisti e perseguitati fuggiti precipitosamente dalla Parigi occupata. I Trentin provvedono all'ospitalità di molti esuli italiani tra cui Emilio e Joyce Lussu e l'ex primo ministro Francesco Saverio Nitti con la moglie.

Nel suo diario Pietro Nenni scrive che la loro casa è «la più accogliente di tutte, [Trentin], la signora e i figlioli fusi in un unico sentimento: resistere» ${ }^{3}$. Con i primi combattenti della resistenza francese, nel Sudovest, Trentin fonda nel 1941 l'originalissimo movimento Libérer et Fédérer, di cui è ispiratore del programma e capo militare della formazione, in contatto con rappresentanti dell'esercito clandestino. Fautore del fronte unico antifascista, è lui - a nome di GL - a sottoscrivere con il PCI e il PSI a Tolosa, nell'ottobre 1941, il primo patto di unità d'azione della resistenza italiana. Nel novembre del '42, con l'occupazione tedesca di tutta la Francia, Silvio è costretto a passare in clandestinità. Poche settimane dopo Bruno è arrestato con alcuni suoi compagni, sottoposto ad un duro interrogatorio e messo in carcere.

${ }^{3}$ P. Nenni, Taccuino 1942, Ed. Avanti!, Milano-Roma 1955. 
Dopo il 25 luglio del ' 43 Silvio chiede ai due figli maschi di rientrare con lui in Italia per la lotta decisiva contro il fascismo. In agosto i due ragazzi lo accompagnano negli incontri con esponenti del controspionaggio francese e dei servizi inglesi a Marsiglia e a Nizza e nel tentativo del passaggio dei Pirenei, poi fallito per un attacco di cuore di Silvio che li costringe a tornare indietro. Rientrano in Italia ai primi di settembre, assieme a Beppa, allorché il governo Badoglio rilascia i passaporti agli esuli. A Tolosa rimane solo Franca, l'unica naturalizzata francese della famiglia non può espatriare.

Appena due giorni dopo l'armistizio dell' 8 settembre, falliti gli incontri a Treviso e a Feltre con i generali Coturri, Loasses e Nasci per trattare la possibilità di organizzare insieme all'esercito la resistenza ai tedeschi, Trentin è già deciso a varare un'organizzazione clandestina armata. Da subito tra i capi del Partito d'Azione e della resistenza nella regione, con Concetto Marchesi ed Egidio Meneghetti dirige le prime sedute organizzative del Comitato di liberazione nazionale per la regione veneta (CLNRV) e fra il 15 e il 25 settembre interviene per porre le fondamenta del comando militare unificato delle forze partigiane nel Veneto. Nelle stesse settimane redige l'Appello ai Veneti guardia avanzata della nazione italiana (pubblicato nel giornale "Giustizia e Libertà» del primo novembre), lanciando la parola d'ordine di «darsi alla macchia», «nella fraternità di una libera federazione di pionieri della nuova Italia» prefigurando come premessa indispensabile per la costruzione del nuovo stato democratico la costituzione federativa dei CLN, non solo come sistema di alleanze, ma in un processo di unificazione sociale. Il 23 ottobre alla proposta di Lussu di entrare nella direzione centrale del CLN, Trentin risponde che il suo posto di battaglia è nel Veneto e non nella capitale.

Bruno e Giorgio sono a fianco del padre nella sua frenetica attività di incontri clandestini. Questa esperienza segnerà per sempre la vita di Bruno, che fino allora aveva cercato di condurre una sua autonoma battaglia per la libertà, in competizione con il padre. Nei giorni successivi all'8 settembre Bruno inizia a scrivere il Journal de guerre, il suo diario di guerra, nella sua lingua madre, il francese. Il 19 novembre 1943 padre e figlio sono arrestati dalla polizia fascista a Padova. Dopo la scarcerazione dovuta all'aggravamento delle condizioni di salute, il 6 dicembre Silvio è ricoverato in ospedale a Treviso e da febbraio a Monastier, assistito quotidianamente da moglie e figli, riuscendo con il loro aiuto a mantenere i contatti con i compagni di lotta, scrivere un ultimo Appello ai lavoratori delle Venezie e redigere un abbozzo di costituzione per l'Italia del dopoguerra su modello di quello per la Francia, scritto un anno prima. Muore il 12 marzo 1944. Le esequie - un carro solitario per le vie deserte di San Donà, per ordine delle autorità fasciste, seguito da Beppa e i figli, accompagnati dal solo amico Camillo Matter - sono l'immagine di questo gruppo famigliare coeso e forte. 
Beppa si mette a disposizione del movimento di liberazione, a fianco di Giorgio che resta con lei nel trevigiano. Dopo la Liberazione a Treviso collabora con il Cln cittadino, come Presidente dell'Ufficio Assistenza Rimpatriati dalla Germania, provvedendo, con il suo abituale spirito pratico, alla raccolta di fondi e vestiario. È tra le fondatrici dell'UDI a Treviso, e poi, trasferitasi a Venezia, del collegio per orfani di partigiani Biancotto. Dopo lo scioglimento del PdA, si iscrive allo PSIUP e, su pressione di Vittorio Foa, accetta di candidarsi nelle liste elettorali. Punto d'incontro di molte memorie, mantiene legami stretti con tutti gli amici di Silvio: Lussu, Valiani, Riccardo Lombardi e accoglie periodicamente nella sua casa i vecchi amici francesi. Nei primi anni Sessanta accetta la proposta di Ada Gobetti di versare al Centro Gobetti le varie carte autografe e scritti di Silvio, una decisione non scontata, presa da ambedue in piena coerenza con le idee e le azioni dei rispettivi uomini - intuendone le profonde affinità - ma anche con le loro stesse idee e azioni di donne combattenti. Dalla forte intesa tra Ada e Beppa nasce così, a metà degli anni Sessanta, presso il Centro studi di Torino il Fondo Trentin. Quando il 17 maggio 1967 Beppa muore, avrà, per sua volontà, esequie civili, semplici, di terza classe. Viene sepolta a S. Donà nella tomba di famiglia, accanto a Silvio (ora raggiunti dai figli Franca e Giorgio).

Giorgio dopo la morte del padre resta nelle formazioni di pianura, commissario politico del battaglione autonomo Rapisardi aggregato alle forze GL collegate alla Brigata Mameli di Treviso; organizza aviolanci, esegue sabotaggi alle linee di comunicazione, partecipa a numerose azioni nella zona. Nel biennio 1946-48 è segretario del Fronte della Gioventù a Treviso, segretario dell'ANPI e dirige il Partito d'Azione locale. Nel 1948 entra come assistente tecnico alla direzione delle Belle arti del Comune di Venezia. Dal '49 risiede a Venezia, dove sarà per vent'anni presidente dell'ANPI e successivamente, fino alla morte, dell'ANPPIA (Associazione perseguitati politici antifascisti italiani) di Venezia. Diventa un importante critico d'arte e organizzatore culturale e il massimo esperto dell'arte incisoria a livello nazionale. Muore a Venezia il 17 luglio 2013.

Franca continua la sua attività di staffetta nella Resistenza tolosana, fino alla liberazione della città. Il 2 marzo 1944 sposa un rifugiato spagnolo, Horace Torrubia, eroe della guerra civile spagnola e ora dirigente della resistenza nel sud-ovest francese. Apprende dopo varie vicissitudini dell'avvenuta morte del padre e riuscirà a riabbracciare la famiglia soltanto dopo la liberazione dell'Italia. Nel 1946 riceve la decorazione della Resistenza francese, La Croix de la Résistance. Dopo la guerra rimane in Francia con il marito e il figlio primogenito Silvio. Dal 1957 è docente presso il Dipartimento d'Italiano della Sorbona fino al 1966, allorché si trasferisce con il secondo marito, Mario Barat- 
to, a Venezia, dove insegna in qualità di lettrice di lingua e letteratura francese all'Università di Ca' Foscari, continuando fino alla morte nel suo impegno politico e civile di intellettuale militante, fondatrice di molteplici associazioni culturali e promotrice di molte iniziative soprattutto legate al movimento delle donne e all'antifascismo. Dal 1996 al 1999 è presidente dell'Istituto veneziano per la storia della Resistenza e della società contemporanea. Dona il suo archivio all'Associazione per la storia e la memoria delle donne "rEsistenze". Muore a Venezia il 28 novembre 2010.

Bruno, dopo un primo periodo a fianco del fratello nelle formazioni di pianura, partecipa alla resistenza nelle Prealpi trevigiane durante tutta l'estate del 1944; in ottobre, non ancora diciottenne, viene destinato al Comando regionale lombardo. A Milano dirige i Gap di Giustizia e Libertà della città e svolge un ruolo determinante nei giorni dell'insurrezione al comando della brigata partigiana Rosselli. Dopo la guerra partecipa in qualità di delegato nazionale dell'organizzazione giovanile del Partito d'Azione a congressi e convegni in vari paesi europei e negli Stati Uniti dove, per un periodo, con l'aiuto di Gaetano Salvemini, frequenta anche l'università di Harvard. Alla Facoltà di giurisprudenza a Padova segue le lezioni di Norberto Bobbio e di Enrico Opocher con cui si laurea nel '49 con una tesi dal titolo Giudizio di equità nei rapporti commerciali, con particolare riferimento alla dottrina della Corte Suprema degli Stati Uniti. Alla fine del 1949 chiamato da Vittorio Foa entra a lavorare nell'Ufficio studi della Cgil, in contatto diretto con Giuseppe Di Vittorio, segretario generale della Cgil. Da lì inizierà la sua vita tutta spesa «al servizio della classe lavoratrice», come amava dire. L'anno successivo si iscrive al Partito Comunista Italiano, eletto dapprima consigliere comunale a Roma e quindi deputato. Nel 1958 è vicesegretario della CGIL, dal 1962 al 1977 è segretario dei sindacati metalmeccanici FIOM e FLM: in questo periodo è ideatore dei Consigli dei delegati di fabbrica, le nuove forme di rappresentanza collettiva nei luoghi di lavoro in luogo delle vecchie Commissioni interne, e dell'unità della confederazione dei metalmeccanici. Dal 1988 al 1994 è segretario generale della CGIL. Dal 1994 dirige l'ufficio programmi della CGIL. Dal 1999 al 2004 è parlamentare europeo tra le file dei Democratici di Sinistra. Oltre a dirigente sindacale è stato anche un grande intellettuale, europeista convinto, erede per molti versi del pensiero e del temperamento del padre ${ }^{4}$. Muore a Roma il 23 agosto 2007.

${ }^{4}$ Per un approfondimento della figura di Bruno Trentin e del rapporto con il padre si veda: I. Ariemma, La sinistra di Bruno Trentin. Elementi per una biografia, Ediesse, Roma 2014. 


\section{Bibliografia}

Cortese F., Libertà individuale e organizzazione pubblica in Silvio Trentin, FrancoAngeli, Milano 2008. Rosengarten F., Silvio Trentin dall'interventismo alla Resistenza, Feltrinelli, Milano 1980.

Guerrato M., Silvio Trentin un democratico all'opposizione, Vangelista, Milano 1981.

Verri C., Guerra e libertà. Silvio Trentin e l'antifascismo italiano (1936-1939), XL Edizioni, Roma 2011.

$<$ http://www.centrotrentin.it/famiglia-trentin.html > 


\title{
NORBERTO BOBBIO, SILVIO TRENTIN E IL CENTRO STUDI PIERO GOBETTI ${ }^{\star}$
}

\author{
Frank Rosengarten
}

De Luna divenne un professore di Storia Contemporanea all'Università di Torino e anche uno scrittore che fece buon uso del materiale documentario che consultò mentre lavorava al Centro Gobetti. Il suo lavorò più noto è Storia del Partito d'azione 1942-1947, che è stato aggiornato e ristampato più volte in nuove edizioni dopo la sua prima comparsa nel 1982 .

Le mie conversazioni con Giovanni sono state sempre esperienze intense, perché lui cercava le sue verità, come io cercavo le mie; nonostante le differenze, abbiamo trovato un terreno comune. Al tempo lui era coinvolto in scioperi e proteste e mi spingeva ad unirmi a lui in una di queste battaglie. Mi sono rifiutato di farlo, per precauzione, e riluttanza a partecipare ad azioni per le quali non mi sarei sentito coinvolto come invece erano coinvolti coloro che partecipavano a tali azioni in Italia.

In realtà avevo preso parte ad azioni simili a Cleveland, avevo marciato in alcune dimostrazioni, e avevo dato il mio contributo a dibattiti infuocati negli ultimi anni della Guerra del Vietnam, ma non ero preparato a fare cose simili in Italia. Tutto ciò aveva qualcosa di ironico perché se io avevo avuto un'esperienza di protesta negli anni Sessanta (negli States), questo era certamente dovuto alla mia esperienza italiana ed era ciò che scaturiva dalla rabbia che io provavo nei confronti della politica governativa degli USA.

Giorgio e Lori Sesia erano altre persone che avevo conosciuto e con le quali ero entrato in confidenza al Centro Gobetti. In breve tempo, Lilian e io scoprimmo di avere molto in comune con loro e quindi passammo alcune serate piacevoli a casa loro a Torino. Giorgio era un rappresentante di diverse industrie italiane e si recava spesso in Urss per motivi di lavoro che lo portavano ad aver contatti con i russi, molti dei quali gli parlarono delle difficoltà incontrate da quelle persone che non erano allineate con la linea del governo (sovietico). Grazie a Giorgio io

* F. Rosengarten, Through Partisans Eyes. My Friendships Literarry Education and Political Encounters in Italy (1956-2013), Firenze University Press, Firenze 2014, pp. 5253, trad. it. di F. Somenzari. 
fui consapevole, ancora una volta, del problema che mi aveva afflitto per anni, e cioè il problema della coesistenza della mia istintiva simpatia per l'URSS con la realtà delle cose e della vita reale in Russia. Non visitai l'Urss fino al 1983, quindi tutto quello che avevo pensato e dicevo su questo paese era basato su libri e resoconti di giornalisti e storici, che io avevo selezionato attentamente per essere sicuro di non imbattermi in letture che potessero contraddire completamente quello che io pensavo. Il modo di Giorgio di interpretare le sue esperienze in URSS non era del tutto negativo nei toni, perché lui era una sorta di compagno di viaggio. Certamente non poteva non ricordare alcuni momenti di vera difficoltà e rabbia relativamente ad alcune cose che vide lì durante i suoi viaggi di lavoro. In maniera gentile e sottile, egli cercò di forzare la resistenza che io dimostravo nel non criticare apertamente quel paese. Lui mi lasciò una traccia permanente di disillusione e rancore nei confronti di un paese e di un popolo che, sin dall'adolescenza, avevo considerato come un faro di speranza per l'umanità.

A Marvin Becker piace citare il detto kantiano "osa sapere"! parole che fui costretto ad ascoltare e a studiare più e più volte, tanto ostinatamente attaccato ero alle mie illusioni e mistificazioni. Questo però non significa che mi sto preparando a gettare tutto ciò che concerne il socialismo reale in un cassonetto dell'oblio. Infatti non ho ancora intenzione di ripudiare l'intera storia dell'URSS. Mi richiamo a quegli eventi e battaglie dove - secondo me - il paese di Lenin e Trotsky, se non Stalin, ha avuto un ruolo chiave nella difesa della libertà dall'oppressione. [...]

Tra le persone che incontrai a Torino, il cui pensiero e la cui personalità mi colpirono molto ci fu sicuramente il filosofo del diritto Norberto Bobbio. La lettura dei suoi splendidi e vividi ritratti di Italiani che si distinsero nella lotta al Fascismo nel libro intitolato Italia Civile (1964), fu una delle esperienze che resero i miei soggiorni a Torino nel 1965 e 1968 davvero belli e degni di essere ricordati.

Il mio primo incontro con Bobbio avvenne poco dopo il mio arrivo a Torino. Carla Gobetti me lo presentò subito dopo una conferenza che lui tenne su un lavoro iniziale di Kelsen relativo alla teoria di Dante sullo stato. Era una tematica insolita nell'ambito dei dibattiti del Centro Gobetti, abbastanza lontana dal "flusso" dei dibattiti giuridici e politici del momento, ma Bobbio riuscì a rendere il tutto rilevante dal momento che lui vedeva delle connessioni tra la lettura kelsiana di Dante e i dibattiti del momento sulle origini del Fascismo e le concezioni comuniste dello stato.

Mi resi subito conto che Bobbio era un uomo semplice, senza alcuna presunzione (non si dava arie), e allo stesso tempo lucidissimo e del tutto sincero nell'esprimere le sue opinioni, dotato di un senso dello humour che conferiva al suo modo di parlare un che di piacevolmente piccante. Trovammo un terreno comune nel capitolo che Bobbio aveva dedicato 
in Italia Civile al giurista Trentin, la cui vita e il cui lavoro mi avrebbero occupato per il successivo decennio. Negli incontri successivi, al Centro Gobetti o a casa sua a Torino, il marchio di Trentin come socialista rivoluzionario divenne un tema ricorrente delle nostre conversazioni perché questo aiutava entrambi (io e lui) a chiarire le nostre visioni intorno alla relazione esistente tra il socialismo e la democrazia. A me mancava la formazione filosofico- giuridica di Bobbio e non pensai neanche per un momento che il mio discorso sulla teoria democratica potesse essere paragonato a ciò che lui diceva nelle nostre conversazioni; semplicemente lui ascoltava con interesse quello che io avevo da dire e accettava le nostre differenze di opinione come uno stimolo per una continua riflessione. Fui lusingato dal suo amichevole accoglimento delle mie idee.

Ciò che aveva influenzato la formazione giovanile di Bobbio e che mi interessava molto fu la sua amicizia con alcuni degli intellettuali torinesi di sinistra degli anni Trenta e Quaranta, incluso quello che poi divenne un leader sindacale, Vittorio Foa, lo scrittore Cesare Pavese e il pensatore politico Leone Ginzburg, tutte persone che, come Bobbio, erano stati allievi di Augusto Monti negli anni Trenta. Monti seppe riconoscere le qualità e le doti dei suoi studenti, diventando quindi lui stesso una sorta di ponte nella storia dell'anti-fascismo nel capoluogo piemontese.

Ero molto interessato a Pavese, perché avevo studiato e a mia volta spiegato molte delle sue novelle, una delle quali, La Luna e i falo, aveva contribuito alla nascita e al successo della narrativa e della filmografia italiana durante e dopo la Seconda guerra mondiale.

Come ho sottolineato prima, nel 1965 stavo lavorando al Centro Gobetti quando m'imbattei negli scritti di un professore di diritto antifascista italiano che per il ruolo avuto nei movimenti di resistenza francese e italiana dal 1926 alla sua morte nel 1944 mi assorbì intellettualmente ed emotivamente per molti anni. Il suo nome era Silvio Trentin. Anche oggi, 45 anni dopo la mia iniziale reazione ai suoi scritti e ai vari gruppi politici e sociali con i quali egli era in rapporti, non posso fare a meno di ricordare e rivivere la frenesia che mi prese negli anni Sessanta. Ero per natura una persona facilmente impressionabile e sensibile, ma questo era qualcosa di diverso. 



\section{BIBLIOGRAFIA \\ DEGLI SCRITTI DI E SU SILVIO TRENTIN}

La presente guida bibliografica riprende e aggiorna i repertori compresi in N. Bobbio, Ricordo di Silvio Trentin, Stampa Arti Grafiche Sosteni, Venezia 1955; S. Trentin, Scritti inediti. Testimonianze e studi, a cura di P. Gobetti, Guanda, Parma 1972, pp. 319-333; F. Rosengarten, Silvio Trentin dallinterventismo alla Resistenza, Feltrinelli, Milano 1980, pp. 219-238; M. Guerrato, Scritti di Trentin (1907-1926), in Id., Silvio Trentin democratico all'opposizione, Guanda, Milano 1981, pp. 211-222 (poi in S. Trentin, Politica e amministrazione. Scritti e discorsi 1919-1926, a cura di M. Guerrato, Marsilio, Venezia 1984, pp. 395-406).

Gli studiosi dispongono ora di uno strumento prezioso e importante: le Opere scelte di Silvio Trentin, di cui si dà notizia nella prima sezione di questa bibliografia.

Nella seconda sezione Scritti vengono indicate in ordine cronologico le opere di Trentin nell'edizione originaria, segnalandone le riprese successive; si dà inoltre notizia degli scritti postumi, delle traduzioni e delle ristampe successive.

Infine nella terza sezione Studi si informa sulla letteratura secondaria. Qui vengono indicate le monografie, gli atti dei convegni a lui dedicati, i principali saggi. Inoltre viene ripetuta l'indicazione delle introduzioni alle Opere scelte, che costituiscono preziosi contributi alla conoscenza di Trentin.

Lo studioso può avvalersi della documentazione conservata presso l'Archivio Centrale dello Stato, Roma, il Centro Studi Piero Gobetti, Torino, il Fondo Silvio Trentin, presso la Biblioteca civica del Comune di Jesolo, il Centro Documentazione e Ricerca Famiglia Trentin, Venezia e il Fondo Franca Trentin Archivio rEsistenze, Casa della Memoria, Venezia. Sul Centro di Jesolo si veda M. Guerrato, I trent'anni del Centro Studi e Ricerca "Silvio Trentin" (1974-2004), in Id. (a cura di), L'antifascismo italiano tra le due guerre: alla ricerca di una nuova unità, Atti del Seminario di studi italo-francese, introduzione di G. Paladini, Jesolo 2-3 aprile 2004, Centro Studi e Ricerca "Silvio Trentin", Jesolo 2005, pp. 29- 42 e M. Polo e R. Franchin, Il Centro Studi e ricerca "Silvio Trentin": lo stato attuale e le prospettive future, in Pensare un'altra Italia. Il progetto politico di Silvio 
Trentin, Atti del Convegno, Teatro Eden, Treviso, 15 giugno 2011, Istreco-Isever, Treviso-Venezia 2012, pp. 105-115; per i documenti custoditi a Roma: G. Fioravanti, Silvio Trentin nelle carte dell'Archivio centrale dello Stato, in Silvio Trentin e la Francia, Marsilio, Venezia 1991, pp. 57-78; per quelli conservati a Torino: C. Malandrino, Silvio Trentin pensatore politico antifascista, rivoluzionario, federalista, Lacaita, Manduria 2007, pp. 53-85.

Sulla storia delle carte trentiniane 'salvate', tra Venezia e Torino, per la cura premurosa, prima di Beppa Nardari Trentin e Ada Gobetti, poi di Franca Trentin e di Carla e Paolo Gobetti, vedi il contributo di Silvana Barbalato, Note sull'eredità di Silvio Trentin tra Venezia e Torino, in F. Cortese (a cura di), Liberare e federare. L'eredità intellettuale di Silvio Trentin, Firenze University Press, Firenze 2016, pp. 267-270.

\section{Opere scelte}

Dallo statuto albertino al regime fascista, a cura e traduzione di A. Pizzorusso, Marsilio, Venezia [ottobre] 1983, pp. XXXI-417.

Politica e amministrazione. Scritti e discorsi 1919-1926, a cura di M. Guerrato, Venezia [novembre] 1984, pp. LVII-410. Con una Appendice bibliografica: Scritti di Trentin (1907-1926), pp. 395-406. D'ora in poi: PA.

Antifascismo e rivoluzione. Scritti e discorsi 1927-1944, a cura di G. Paladini, Marsilio, Venezia [gennaio] 1985, pp. 34-534. D'ora in poi: AR.

Federalismo e libertà. Scritti teorici 1935-1943, a cura di N. Bobbio, Marsilio, Venezia [gennaio] 1987, pp. XXXVII-398. D'ora in poi: FL.

Diritto e democrazia. Scritti sul fascismo 1928-1937, a cura di G. Paladini, introduzione di A. Ventura, Marsilio, Venezia [novembre] 1988, pp. LII-286. D'ora in poi: DD. L'introduzione è compresa in A. Ventura, Intellettuali. Cultura e politica tra fascismo e antifascismo, introduzione di E. Gentile, Donzelli, Roma 2017.

Libérer et Fédérer: 14 juillet 1942-avril-mai 1944, présentation de Michel Dreyfus, publié avec le concours des municipalités de Venise et de Jesolo et du Centro di ricerca Silvio Trentin.

\section{Scritti}

La cosa giudicata nelle decisioni delle sezioni giurisdizionali del Consiglio di Stato, Nistri, Pisa 1910, pp. 266.

La responsabilità collegiale, prefazione di G. Vacchelli, Vallardi, Milano 1910, pp. XVI-490.

L'atto amministrativo: contributi allo studio della manifestazione di volontà della pubblica amministrazione, Athenaeum, Roma 1915, pp. 27.

Appunti di diritto amministrativo, lezioni tenute all'Università di Padova, raccolte dallo studente P. Compostella, anno accademico 1915-1916, Padova 1916. 
Per un nuovo orientamento della legislazione in materia di bonifiche in rapporto alle presenti esigenze dell'economia nazionale, Cooperativa Casa del Popolo, Venezia 1919, pp. 36.

Gli enti pubblici del Veneto di fronte al problema delle utilizzazioni idrotecniche, Officine Grafiche e Commerciali C. Ferrari, Venezia, 1922, pp. 377. La prefazione e i primi due capitoli sono riprodotti in PA, pp. 197-261.

Corso di istituzioni del diritto pubblico: appunti alle lezioni (1923-1926), La litotipo ed. universitaria, Padova 1926.

La restaurazione delle terre liberate in Italia e l'opera dell'Istituto federale di credito per il risorgimento delle Venezie, Zanichelli, Bologna 1923, pp. 95. Vedilo ora in PA, pp. 263-329.

Autonomia - autarchia - decentramento, Officine Grafiche e Commerciali C. Ferrari, Venezia 1925, pp. 25-83. Vedilo ora in PA, pp. 335-374.

L'aventure italienne. Légendes et réalités, Préface di M. A. Aulard, Les Presses Universitaires de France, Paris 1928, pp. 332. Le pp. 13-20, 23-44, 109145 e 195-210 sono riprodotte in DD, pp. 3-63. Traduzione di D. Orati.

Les transformations récentes du droit public italien. De la Charte de Charles-Albert à la creation de l'État fasciste, M. Giard, Paris 1929, pp. 440. $25^{\circ}$ volume della Bibliothèque de l'Institut de droit comparé de Lyon, diretta da E. Lambert, prefazione di Julien Bonnecase, professore della facoltà giuridica di Bordeaux, con un'appendice dei principali testi legislativi. Trad. it. a cura di A. Pizzorusso, Dallo statuto albertino al Regime fascista, Marsilio, Venezia 1983, pp. 3-408, con l'omissione della prefazione e dell'appendice.

Antidémocratie, Valois, Paris 1930, pp. 279. Le pp. 30-52 e 261-275 sono riprodotte in AR, pp. 21-53. Traduzione di P. Ulvioni; le pp. 195-259 sono riprodotte in DD, pp. 85-139. Traduzione di D. Orati.

Oì rechercher les supports d'une internationale européenne, Valois, Paris 1930. Vedilo ora in AR, pp. 68-94.

Aux sources du fascisme, M. Riviére, Paris 1931, pp. 212. Le pp. 105-129 e 133149 sono riprodotte in DD, pp. 143-172.

La libertà e le sue guarentigie, "Liberissima», III (1), Paris 1932. Vedilo ora in AR, pp. 67-97.

Le fascisme à Genève, M. Riviére, Paris 1932, pp. 262. Le pp. 21-33, 35-46, 89-107, 139-167, 243-255 sono riprodotte in DD, pp. 175-216.

Le code pénal fasciste, Ligue Italienne des Droit de l'Homme, Paris1932.

La mystification de l'amnistie fasciste, "Problemi della rivoluzione italiana», Marsiglia, Quaderno XV-XVI, gennaio 1933, pp. 27. Vedilo ora in AR, pp. 217-247. Traduzione di P. Ulvioni.

Riflessioni sulla crisi e sulla rivoluzione, Edizioni Sala "Italia Libera" (ESIL), Marseille, senza data [ma 1933], pp. 155. La prima parte, pp. 3-21 e l'ultima, pp. 82-155 sono riprodotte in AR, pp. 111-216.

Sugli obiettivi della rivoluzione italiana, "Problemi della rivoluzione italiana», Marsiglia, 21-22, marzo 1934, pp. 6-27. Vedilo ora in AR, pp. 251-273.

Bisogna decidersi, "Quaderni di Giustizia e Libertà», 11, giugno 1934, pp. 99108. Vedilo ora in AR, pp. 275-286. 
L'Unione Sovietica e la Società delle nazioni, «Problemi della rivoluzione italiana», Marsiglia, 27-28, novembre 1934, pp. 26.

La Crise du Droit et de l'État, préface de F. Geny, Alcan, Paris-Bruxelles 1935, pp. 498 (L'Eglantine). Il cap. VII - Capitalismo e fascismo, pp. 418-428, è riprodotto in DD, pp. 219-228. Il cap. VIII - ultimo del volume, pp. 445-479, è riprodotto in FL, pp. 3-31. Trad. it., La crisi del diritto e dello stato, a cura di G. Gangemi, Gangemi Ed., Roma 2006; con un saggio del curatore, Silvio Trentin, il diritto naturale e la libertà come autonomia, pp. 7-40.

[Commento alla Costituzione dell'U.R.S.S. del 1936], "Giustizia e Libertà», III (27-28-29), Paris, 3, 10, 17 luglio 1936, rispettivamente pp. 1, 2 e 2. Ora in AR, pp. 305-320.

Dix ans de fascisme totalitaire en Italie. De l'installation du tribunal special à l'établissement de l'empire, Edit. Soc. Internationales, Paris 1937, pp. 244. Trad. it., Dieci anni di fascismo totalitario in Italia, prefazione di E. Santarelli, Editori Riuniti, Roma 1975. I paragrafi corrispondenti nella edizione italiana alle pp. 133-195 e 205-210 sono riprodotti in DD, pp. 231-279.

Lauro De Bosis, chantre et héros de la liberté, Commemorazione tenuta a Tolosa su iniziativa del "Cercle d'études universitaires", il 19 décembre 1937, Jean Flory, Paris 1939, pp. 95. Vedilo ora in AR, pp. 363-388. Traduzione di P. Ulvioni.

D'un poète qui nous permettra de retrouver l'Italie, Giacomo Leopardi, Stock, Paris 1940, pp. 118. Trad. it., a cura di M. Raccanello, Giacomo Leopardi. Un poeta che ci permetterà di trovare l'Italia, introduzione di D. Casagrande, San Donà di Piave, Città di San Donà di Piave 2003. Le pp. 5-15; 20-27; 4648; 109-118 sono riprodotte in AR 505-520. Traduzione di P. Ulvioni.

Stato - Nazione - Federalismo, prefazione di M. Dal Pra, «La Fiaccola», Milano 1945, "Biblioteca di cultura politica", n. 1, (edizione clandestina ma uscita dopo la Liberazione), pp. 8-214. Questo volume è interamente riprodotto in FL, pp. 5-231. Segnalo l'edizione anastatica per iniziativa del Consiglio Regionale del Veneto, Marsilio, Venezia 2010.

Scritti inediti. Testimonianze. Studi, contributi di E. Lussu e H. W. Tobler, a cura di P. Gobetti, Guanda, Parma 1972.

Comprende:

- L'abdicazione della Francia o la fine di un mondo. Note di un sopravvissuto, 1 ottobre 1940, pp. 107-186;

- Liberare e federare. Dare un senso e uno scopo alla rivoluzione europea in corso di gestazione, 1943, pp. 189-278. Il libro è stato scritto in francese, con il titolo di Libérer et fédérer, nel 1942. Il testo compreso in Scritti inediti e riprodotto in FL, pp. 235-321, è quello tradotto da A. Giuriolo sul manoscritto trentiniano nel 1943;

- Ébauche de la figure costitutionelle de la France à l'issue de la Révolution en cours de développement, pp. 279-293. Trad. it., a cura di D. Orati, Abbozzo di un piano tendente a delineare la figura costituzionale della Francia al termine della rivoluzione federalista in corso di sviluppo, in FL, pp. 325-339; Abbozzo di un piano tendente a delineare la figura costituzionale dell'Italia al termine della rivoluzione federalista in corso di sviluppo, pp. 295-318; ora in FL, pp. 341- 
360 e in «Storia Amministrazione Costituzione, Annale dell'Istituto per la Scienza dell'Amministrazione Pubblica», 25, 2017, pp. 7-24, con una nota di D. Cadeddu.

Le determinanti dialettiche e gli sbocchi ideologici e istituzionali della rivoluzione antifascista [europea]. Saggio inedito del 1944, a cura e con introduzione di C. Malandrino, prefazione di M. Revelli, Lacaita, Manduria-Bari-Roma 2007, pp. 244. Il saggio di Trentin è alle pp. 75-244. Il manoscritto è conservato nel Fondo Trentin del Centro studi Piero Gobetti.

\section{Studi su Silvio Trentin}

Arrighi P., Silvio Trentin, un Européen en Résistance (1919-1943), Loubatières, Portet-Sur-Garonne 2007.

Atti del Convegno comprensoriale di studi su Silvio Trentin, a cura del Centro di ricerca "Silvio Trentin" per la documentazione e lo studio sull'antifascismo e la Resistenza nel Veneto, Biblioteca Comunale di Jesolo, 31 marzo 1974, pp. 36.

Atti del convegno di studi su Silvio Trentin, Jesolo, 20 aprile 1975, Neri Pozza, Vicenza 1976.

Biason M., Un soffio di libertà. La Resistenza nel basso Piave, Nuova Dimensione, Portogruaro 2007.

Biondo R., Borghi M. (a cura di), Giustizia e libertà e Partito d'azione a Venezia e dintorni, Nuova Dimensione, Portogruaro 2005.

Bobbio N., Silvio Trentin, in Italia civile. Ritratti e testimonianze, ManduriaBari-1964, pp. 271-288; seconda ed. Passigli, Firenze 1996, pp. 249-266.

Bobbio N., La crisi europea e lo stato federale nell' opera di Silvio Trentin, "Città e Regione», I (8), ottobre 1975, pp. 200-212. Discorso al Convegno, Jesolo 20 aprile 1975.

Bobbio N., Silvio Trentin e lo stato fascista, «Belfagor», XL (6), 30 novembre 1985, pp. 700-707.

Bobbio N., Introduzione, in FL, S. Trentin, pp. IX-XXXVII.

Bobbio N., François Gény: una prefazione, «Quaderni per la storia del pensiero giuridico moderno», 20, 1991, pp. 181-187.

Cadeddu D. (a cura di), Dalla libertà al federalismo. Silvio Trentin tra storia e teoria politica, Biblion edizioni, Milano 2010.

Cadeddu D., La Repubblica federale di Silvio Trentin, "Storia Amministrazione Costituzione, Annale dell'Istituto per la Scienza dell'Amministrazione Pubblica», 25, 2017, pp. 25-40.

Casagrande D., Introduzione, in S. Trentin, Giacomo Leopardi. Un poeta che ci permetterà di trovare l'Italia, a cura di M. Raccanello, San Donà di Piave, Città di San Donà di Piave 2003, pp. 5-16.

Cisotto G.A., "Solo uomini di buona volontà". Il Partito d'azione veneto (19421947), Viella, Roma 2014.

Cortese F., Libertà individuale e organizzazione pubblica in Silvio Trentin, FrancoAngeli, Milano 2008. 
Cortese F., Silvio Trentin. Maestro di diritto, apostolo della democrazia, pubblicazione a cura del Centro Documentazione e Ricerca Trentin, 2012, <http://www. centrotrentin.it/testi-e-materiali-online/articoli.html> (12/2019).

Cortese F. (a cura di), Liberare e federare. L'eredità intellettuale di Silvio Trentin, Atti del Convegno tenutosi a Venezia il 5 dicembre 2014, in occasione del settantesimo anniversario della morte, "Carte studi opere Centro Trentin di Venezia”, vol. IV, Firenze University Press, Firenze 2016.

Cortese F. (a cura di), Resistenza e diritto pubblico, Atti del Convegno tenutosi a Venezia, presso Iveser (Istituto veneziano per la Storia della Resistenza), il 30 maggio 2014, "Carte studi opere Centro Trentin di Venezia", vol. III, Firenze University Press, Firenze 2016.

Feltrin F., Nuovi documenti su Silvio Trentin. L'arresto e la detenzione a Padova (19 novembre-2 dicembre 1943), in Istituto veneto per la storia della Resistenza e dell'età contemporanea, Annale XX, Cleup, Padova 1999, pp. 9-88.

Gangemi G., Federalisti contro. Da Althusius a Silvio Trentin, prefazione di D. Fiorot, Edizioni Sapere, Padova 1997.

Gangemi G., Silvio Trentin e Giuseppe Capograssi: similitudini e differenze, «Rivista internazionale di filosofia del diritto", LXXXII (3), 2005, pp. 377-401.

Gangemi G., Silvio Trentin, il diritto naturale e la libertà come autonomia, Introduzione, in S. Trentin, La crisi del diritto e dello stato, Gangemi Ed., Roma 2006, pp. 7-40.

Gangemi G., Ricordo di Silvio Trentin, «Foedus», 18, 2007, pp. 75-77.

Gangemi G., Silvio Trentin e la sua opera più importante: La crisi del diritto e dello stato, «Ritorno al diritto», I (6), 2007, pp. 222-238.

Gangemi G., Bobbio e Trentin tra Resistenza e Filosofia del diritto, in G. Zaccaria (a cura di), Norberto Bobbio. Gli anni padovani, Padova University Press, Padova 2010, pp. 53-92.

Gangemi G., Silvio Trentin: due percorsi per la democrazia e il federalismo, «Rivista di storia della filosofia», LXVII (2), 2012, pp. 361-377.

Guerrato M., Silvio Trentin un democratico all'opposizione, Vangelista, Milano 1981.

Guerrato M., Introduzione, in PA, pp. IX-LV.

Guerrato M. (a cura di), L'antifascismo italiano tra le due guerre: alla ricerca di una nuova unità, Atti del Seminario di studi italo-francese, introduzione di G. Paladini, Jesolo 2-3 aprile 2004, Centro Studi e Ricerca "Silvio Trentin", Jesolo 2005.

Invernici F., Silvio Trentin e la storia come svalutazione del relativismo, «Il Politico», L (1), marzo 1985, pp. 101-116.

Lotto A. (a cura di), Lo stato del federalismo, Atti del Colloquio di storia, Jesolo 1994, introduzione di G. Paladini e A. Ventura, Centro di ricerca Silvio Trentin di Jesolo, Treviso 1998.

Luciani M., L'Italia ritrovata grazie ad un poeta? Politica e forme di governo nel pensiero di Giacomo Leopardi, «Diritto pubblico», 1-2, gennaio-agosto 2010, pp. 55-140.

Lussu E., Profilo di Silvio Trentin, in S. Trentin, Scritti inediti. Testimonianze e studi, a cura di P. Gobetti, Guanda, Parma 1972, pp. 5-23. 
Maddalena M., Rivoluzione, autogestione e federalismo nel pensiero di Silvio Trentin, «Il movimento di liberazione in Italia», ottobre- dicembre 1973, pp. 79-105.

Malandrino C., Introduzione, in Le determinanti dialettiche e gli sbocchi ideologici $e$ istituzionali della rivoluzione antifascista [europea]. Saggio inedito del 1944, prefazione di M. Revelli, Lacaita, Manduria-Bari-Roma 2007, pp. 11-70.

Malandrino C., Silvio Trentin pensatore politico antifascista, rivoluzionario, federalista, Lacaita, Manduria 2007.

Paladini G., Silvio Trentin dalla democrazia radicale al socialismo federalista (19221944), "Archivio Veneto», serie V, CXVI, 1981, pp. 59-83.

Paladini G., Introduzione, in AR, pp. IX-XXXIV.

Paladini G., Introduzione, in S. Trentin, DD, pp. IX-LII.

Pensare un'altra Italia. Il progetto politico di Silvio Trentin, Atti del Convegno, Teatro Eden, Treviso, 15 gennaio 2011, Istreco-Isever-Anpi, Treviso 2012.

Pizzorusso A., Introduzione, in S. Trentin, Dallo statuto albertino al regime fascista, Marsilio, Venezia [ottobre] 1983, pp. IX-XXXIV.

Polito P., La terza via di Silvio Trentin, «Agorà», Annuario del Liceo Scientifico "Galileo Ferraris" di Varese, anno V, 2001, pp. 647-670.

Ronchi V., Silvio Trentin. Ricordi e pensieri 1911-1926, Edizioni Canova, Treviso 1975.

Rosengarten F., Silvio Trentin dall'interventismo alla Resistenza, Feltrinelli, Milano 1980.

Santarelli E., Prefazione, in S. Trentin, Dieci anni di fascismo totalitario in Italia, Editori Riuniti, Roma 1975, pp. 9-32.

Silvio Trentin e la Francia, Colloquio internazionale tenutosi a Parigi l'8 e il 9 febbraio 1985, prefazione di G. Paladini, Marsilio, Venezia 1991.

Tobler H.W., Il pensiero politico di Silvio Trentin, in Trentin, Scritti inediti. Testimonianze e studi, cit., pp. 54-85.

Torlontano G., La vita e L' opera di Silvio Trentin, «Nuova Antologia», 2152, ottobre-dicembre 1984, pp. 40-45.

Verri C., Trentin-Grieco. Un carteggio nel mezzo della guerra d'Etiopia, «Italia Contemporanea», 242, 2006, pp. 95-120.

Verri C., Il primo antifascismo in esilio in una lettera di Trentin a Turati, «Italia Contemporanea», 252-253, 2008, pp. 529-538.

Verri C., Caro Nenni. Appunti per un epistolario di Silvio Trentin, "Annali della Fondazione Ugo La Malfa», 24, 2009, pp. 149-169.

Verri C., Silvio Trentin e Giovanni Colonna di Cesarò. Note sull'antifascismo democratico degli anni Venti, «Venetica», 20, 2009, pp. 77-101.

Verri C., Guerra e libertà. Silvio Trentin e l'antifascismo italiano (1936-1939), XL edizioni, Roma 2011.

Verri C., Le commemorazioni: uno strumento della politica, "Annali della Fondazione Ugo La Malfa», 24, 2011, pp. 227-245.

Verri C., Silvio Trentin e Ivanoe Bonomi: crisi della democrazia, "Italia Contemporanea», 262, 2011, pp. 107-117.

Verri C., Il federalismo antifascista di Silvio Trentin, «Storia e politica», 2, 2012, pp. 250-268. 
Verri C., L'affaire Tasca e la "terza forza": un episodio della guerra fredda, "Annali della Fondazione Ugo La Malfa», 27, 2012, pp. 305-319.

Verri C. (a cura di), I Trentin a Mira nella Resistenza, Atti del Convegno, Mira, ottobre 2011, ANPI, sezione di Mira (Venezia), Mira 2013.

Verri C., Prove di costituzione nella Resistenza, in F. Cortese (a cura di), Resistenza e diritto pubblico, "Carte studi opere Centro Trentin di Venezia", vol. III, Firenze University Press, Firenze 2016.

Verri C., Silvio Trentin, in A. Aghemo, G. Amari, B. Palmieri (a cura di), Preludio alla Costituente, Prefazione di Valdo Spini, postfazione di G. Amato, Castelvecchi, Roma 2018, pp. 359-383.

Zanon Dal Bo A., Silvio Trentin, in Enciclopedia dell'antifascismo e della Resistenza, La Pietra, Milano 1989, vol. VI e Appendice, pp. 115-119. 


\section{CARTE, STUDI E OPERE - CENTRO TRENTIN DI VENEZIA \\ Volumi pubblicati}

1. "Lavoro e conoscenza» dieci anni dopo. Attualità della lectio doctoralis di Bruno Trentin a Ca" Foscari, a cura di Alessandro Casellato, 2014

2. Incidere, Incidere, Incidere. Giorgio Trentin tra etica dell'arte e impegno politico, a cura di Giovanni Sbordone, 2015

3. Resistenza e diritto pubblico, a cura di Fulvio Cortese, 2016

4. Liberare e federare. L'eredità intellettuale di Silvio Trentin, a cura di Fulvio Cortese, 2016

5. Norberto Bobbio, L'esempio di Silvio Trentin. Scritti 1954-1991, a cura di Pina Impagliazzo, Pietro Polito, 2020 
